\title{
Posters
}

\section{Poster Session 1_Case Reports}

\section{P1_1}

Tubo-ovarian abscess in woman with an intrauterine device forgotten for 22 years

D.W. Luedders, N. Chalvatzas, C. Banz, A. Horneman, K. Diedrich, A. Kavallaris

University of Schleswig-Holstein, Campus Luebeck

A 53 year old premenopausal woman was seen as an emergency case in the outpatients' clinic of the Department of Surgery. She had suffered from abdominal pain for five days with fever up to $38^{\circ} \mathrm{C}$ and elevated blood infection parameters. Furthermore, she suffered from obstipation and growth of abdominal girth. Due to the abdominal pain a computed tomography was performed. It showed an unclear structure near the uterus. She was then referred to our department. The last gynaecological examination had been performed 15 years before. In our examination an enlarged painful uterus myomatosus with an orthotropic IUD was suspected, with the IUD inserted 22 years before. The patient was of the opinion that the IUD had been lost during a menstruation long time before because her gynaecologist had been unable to locate it 15 years before. However, the removal of the IUD initially failed in our department due to the lack of a thread. The clinical signs strongly suspected a TAO. First, a conservatively therapy with intravenous antibiotics was started due to a lack of sufficient fasting time then followed by surgical intervention. It showed a tuboovarian abscess (TOA) of the complete pelvis, an enlarged uterus myomatosus with enlarged tubes and a lost orthotopic IUD with a smear positive for E. coli and Candida albicans. Diagnosis was confirmed by histopathological examination. The intravenous antibiotic therapy was continued for further five days with a decline of all blood infection parameters. After one month a total laparoscopic hysterectomy with bilateral adnexectomy was performed in a second-look operation, showing severe adhesions within the complete pelvis. Key-words: tubo-ovarian abscess, lost orthotopic IUD, laparoscopy.
P1_2

Tubo-ovarian abscess following Essure ${ }^{\circledR}$ sterilization L. Barros, R. Gouveia, F. Sousa

Hospital de Dona Estefânia, Portugal

Female surgical sterilization is one of the most frequent method of contraception among women. Since the introduction of Essure ${ }^{\circledR}$ Permanent Birth Control System, the transcervical route has become increasingly popular due to the ease of performance of a less invasive procedure, with minimal anesthesia and decreased complication rate. We report a case of a patient with tubo-ovarian abscess (TOA) 3 months after the insertion of the Essure device. It was a 34 year old patient, G2P2, with no relevant past history, admitted in our department for permanent contraception. Placement of the microinserts was satisfactory, in an ambulatory setting, under no anesthesia and the patient remained on alternative contraception until an abdominal $\mathrm{X}$-ray was performed 3 months after the procedure that confirmed the proper location of the devices. One week after the confirmation test she was observed in the emergency department with severe lower abdominal tenderness and a large palpable mass. Additional tests revealed: leucocytosis, elevated C-reactive protein $(40 \mathrm{mg} /$ dl) and a complex mass, with $10 \mathrm{~cm}$, involving both adnexae in the transvaginal sonography. She was hospitalized, initiated intravenous antibiotics and on the 5th day of treatment she was submitted to a laparoscopy and drainage of the abscess. She improved gradually and discharged home 5 days after surgery on oral metronidazol. Despite the gap time between the microinserts placement and the diagnosis of the TOA, this complication may be assigned to the Essure ${ }^{\circledR}$ system since this patient presented no other risk factors for pelvic inflammatory disease and there is a wide variation in the clinical presentation of this entity. Key-words: Surgical sterilization, Essure ${ }^{\circledR}$, tubo-ovarian abscess.

\section{P1_3}

\section{Conservative management of cervical pregnancy}

A.A. Dubinin, A.V. Ignatiev

OAO "Medical Company IDK", Russia 
Introduction: Cervical ectopic pregnancy is a rare lifethreatening condition with a frequency $0.1-0.4 \%$ of all ectopic pregnancy. Cervical ectopic gestation would often present with life threatening haemorrhage and would often require hysterectomy. However with the aid of early ultrasound diagnosis and development of new medical therapy, conservative non-surgical management of this condition is now possible.

Materials and methods: This is a report of six cases successful conservative treatment of cervical pregnancy, determined by TV ultrasound and serum hCG. In 4 cases of 4-5 weeks of gestation (in absence of fetal cardiac activity) the needle aspiration of amniotic fluid has been performed. Then methotrexate $60 \mathrm{mg}$ was administered into gestational sac cavity. One of these cases had been completed with an episode of a light vaginal bleeding. Bipolar cervicoresectoscopy had been performed in order to remove chorionic tissues and for coagulation of gestation sac. Two other cases were 5-6 weeks of cervical pregnancy with detected fetal cardiac activity. One of these cases had being treated with bipolar cervicoresectoscopy. Other patient had treatment with duplex selective temporary embolization of uterine arteries with subsequent vacuum-aspiration of gestation sac. We had used the haemostatic sponge as an embolization substance. Doppler ultrasonography had been performed during next day after embolization for control.

Result: All cases of cervical pregnancy were treated successfully without hysterectomy. One of six patients had a complication of light vaginal bleeding that was resolved with cervicoresectoscopy.

Discussion: Gerardo Vela and Togas Tulandi 2007 described the most cases of treatment of cervical pregnancy. Of the 12 cases of pregnancy in the 5 was treated by methotrexate. In 3 cases the embolization of uterine arteries was used after infusion of methotrexate, in 1 case - curettage of canal of cervix - was not needed another intervention. P.Martinelli et al. (2007) reported about 3 cases and Y.Nakao et al. (2008) reported about 2 cases of embolization of uterine arteries following with subsequent curettage of cervix. Extirpation of uterine can be considered as method of treatment of cervical pregnancy in late reproductive age or later stage of gestation. Therapy of methotrexaty can be used with absence fetal heartbeats. Isolated use the cervicoresectoscopy associated with risk of intra and postoperative bleeding. Better use the temporary embolisation of uterine arteries.

Key-words: cervical pregnancy, selective temporary embolization of uterine arteries, cervicoresectoscopy.

\section{P1_4}

The role of the transcervical embrioscopy to define a case of trisomy 18 suspected by ultrasound imaging and confirmed by embrio chromosomal study (CVS) E. Castellacci, L. Rosignoli, S. Calzolari, E. Periti Palagi Hospital, Florence, Italy

Introduction: the embrioscopy represent a valid diagnostic support to confirm and complete the study of suspected morfological abnormalities diagnosticated by ultrasound imaging.

Materials and methods: a case report of a woman at 10 weeks pregnancy, with an ultrasound suspect of chromosomal abnormalities

Results: the transcervical embrioscopy confirm the presence of embrional signs of chromosomal abnormalities suspected with ultrasound imaging and confirmed by chromosomal exam (chorionic villus sampling)

Discussion: this case report represents a prospective collaboration of the endoscopy unit with the prenatal diagnosis in the application of the transcervical embrioscopy to confirm the suspect of ultrasound imaging abnormalities and the direct visualization of the embrional morphological abnormalities until $12+6$ weeks.

Key-words: embrioscopy, office hysteroscopy, chromosomal abnormalities.

\section{P1_5}

Excessive production of ascitic fluid after laparoscopic operation for early stage ovarian cancer

D. Evaggelinos $^{(1)}$, I. Kotsopoulos ${ }^{(1)}$, P. Skafida ${ }^{(1)}$, V. Kartsiounis $^{(1)}$, N. Chalvatzas ${ }^{(2)}$, A. Kavallaris ${ }^{(2)}$

(1) Gynaecologic Oncology Department, "Theagenio" Anticancer Hospital of Thessaloniki, Greece.

${ }^{(2)}$ University of Schleswig-Holstein, Campus Luebeck, Department of Gynaecology and Obstetrics, Luebeck, Germany

Introduction: Postoperative production of ascitic fluid after operations for advanced ovarian cancer is not rare. However, in early stage (Ia) ovarian cancer, postoperative ascites, usually can't be cancer related. In this paper, a rare complication of excessive production of ascitic fluid after laparoscopic operation and the used treatment method are presented.

Case Report: Authors present a case of a 27-year-old woman with history of clear cell ovarian carcinoma diagnosed through histopathological examination of a cystic tumor from the left ovary. A second look operation 
was decided due to histological type of the tumor and the patient underwent laparoscopic hysterectomy, bilateral salpingo-ophorectomy, omentectomy, bilateral external ilium lymphadenectomy, using ultrasound knife, and staging. The deduction of the pathological examination indicated no residual malignancy. During the operation there was thermal injury of the obturator nerve. Suturing of the nerve through laparotomy was immediately operated. Postoperative motility disorders were totally restored in 10 days. However, there was an excessive production of ascitic fluid starting from postoperative day 5. Fluid's cytological and microbiologic controls were negative and biochemical examination indicated transudate. Until postoperative day 53, about 21 lit. of ascitic fluid were drained and no applied treatment was able to control the production. There was also production of pleural fluid causing respiratory distress. A second laparoscopy was decided performing by specialist in laparoscopic oncological surgery. During the operation a pseudo-membrane covering entire peritoneal cavity was found and extracted. Also, ascitic fluid was drained and adhesiolysis was operated. Patient was discharged home on postoperative day 7 without complications and with no more ascitic or pleural fluid production. Authors consider that pressure from the pseudo-membrane to hepatic vessels was the main factor causing this rare complication. On the other hand, possible infection could also be accused, but the use of antibiotics combination caused no indication of infection in fluid analysis.

Conclusions: Laparoscopic omentectomy is feasible. In addition, although the use of ultrasound knife can improve laparoscopic operation, there is still danger of thermal injuries. Finally, formation of pseudo-membranes and excessive production of ascitic fluid due to compression of hepatic vessels from these membranes or from adhesions could be very rare postoperative complications. Adhesiolysis and ascites drainage seems to be an indicated treatment procedure. Key-words: ovarian cancer, excessive production of ascitic fluid, laparoscopy.

\section{P1_6}

Retained products of conception at the site of a previous caesarean section scar mimicking submucosal leiomyoma in ultrasonography. A Case Report

K. Myounghwan, Y. Myeongjin

Sanggye Paik Hospital, South Korea

Study Objective: To report the experience of hysteroscopic removal of placental remnant at the site of a previous caesarean section scar mimicking submucosal leiomyoma in ultrasonography in a patient who com- plained of pelvic pain. Design: Case report. Setting: Private clinic. Patients: A 38-year-old woman, gravida 2 caesarean section 1, presented with pelvic pain. This symptom started several months ago and made her difficult to live a daily life. She had a caesarean section 2 years ago and dilatation and curettage due to missed abortion 6 months ago. She had no febrile or chilling sensation. Pelvic exam revealed mild uterine and adnexal tenderness. Transvaginal ultrasonography(TVU) showed an anteverted uterus with diameter $3 \mathrm{~cm}$ sized spherical mass placed in the lower anterior wall suggesting submucosal leiomyoma. $\mathrm{CBC}$ was within normal range and negative beta-hCG was noted. Hysteroscopy was done. Hysteroscopy showed $3 \mathrm{~cm}$ sized purplish colored irregular contour mass in the right lower anterior uterine segment and removed by suction and resection. Pathologic exam demonstrated placental remnant. Her pelvic pain was dramatically resolved after that.

Intervention: Hysteroscopic removal . Measurements and Main Results: N/A

Conclusion: This case shows that previous caesarean section scar may be the cause of pelvic pain due to impaction after D\&C. Hysteroscopy is useful tool to make a differential diagnosis and treat the abnormal mass at the site of previous caesarean section scar.

Key-words: Caesarean section scar, hysteroscopy, pelvic pain.

\section{P1_7}

Scar endometriosis presenting as an acute abdomen and excised laparoscopically

H.J. Lee, H.W. Lee

Daegu Fatima Hospital, Korea

Introduction: Endometriosis is a frequent clinical problem in women of reproductive age and can markedly influence both reproductive prognosis and quality. Scar endometriosis is relatively rare, typically occurring in women with a history of caesarean section. Nowdays, caesarean section incidence is rising and scar endometriosis could be encountered more in the day to day clinical practice.

Materials and methods: We report a case of scar endometriosis presenting as acute abdomen without classical symptoms, so misdiagnosed as other diasease. We excised completely using laparoscopy because the lesion located at peritoneum and for examination of other intraabdominal problem. After surgery, the acute abdomen was disappeared. 
Conclusions: Scar endometriosis can be presented with variable symptoms. If the patients with acute abdominal pain have a history of caesarean section or other gynaecologic surgery and abdominal superficial mass, we have to rule out abdominal scar endometriosis.

Key word: Sacr endometriosis.

\section{P1_8}

Pelvic tuberculosis in gynaecology: laparoscopic findings F. Lo Cane, O. Sizzi, A. Rossetti, A. Loddo Nuova Villa Claudia, Roma, Italy

Peritoneal tuberculosis (TB) is still a major health problem in developing countries in young women between 15 and 25 years of age. It can be easily confused with peritoneal carcinomatosis and advanced ovarian carcinoma. Pelvic pain and mass, ascites and increased CA 125 levels are well known markers of both ovarian cancer and peritoneal tuberculosis. The fallopian tubes are the first and most commonly affected genital organs, followed by the endometrium, ovary, and cervix. Adhesions between tubes, ovaries, omentum, intestines, liver, and diaphragm (the Fitz-Hugh-Curtis syndrome) are common findings in tuberculosis. Nodularity can be detected on the Douglas pouch secondary to tubercle formation on serosal surfaces of the pelvic organs. A 34-year-old woman, immigrated from Rumania to Italy, with primary infertility, without fever, pain or white blood count elevation, presented to our department. Her past medical history was not significant. Preoperative ultrasonography showed an huge ovarian cyst with regular edges containing fluid. In the pouch of Douglas there was not fluid. Tumor markers were within normal limits. Subsequent laparoscopy revealed the presence of adhesions between sigmoid colon and left adnexum. When dissecting the adnexial cystic mass from the periphery, a creamy material spilled out. During the mobilization of the ovary, we found a little nodule $1 \mathrm{~cm}$ in diameter, containing caseous material. The tube was edematous and looked like a hydrosalpynx. Moreover, it was not patent. We removed the ovary and the tube, along with some prevesical creamy little nodules. The histology revealed the presence of nodules with central necrosis and caseation surrounded by granulomatous reaction of epithelioid and multinucleated giant cells (Langhans) at level of the prevesical space and around the tube. In conclusion, the possibility of peritoneal TB should always be kept in mind while evaluating patients living in endemic areas and who have a pelvic mass, ascites, and high CA-125 levels.

Key-words: tuberculosis, laparoscopy, Langhans cells.

\section{P1 9}

\section{Laparoscopic treatment of benign multicystic mesothelioma}

A. Loddo, O. Sizzi, A. Rossetti, F. Torcia, F. Lo Cane

University of Cagliari, Cagliari; Nuova Villa Vlaudia, Roma, Italy

Benign cystic mesothelioma is a very rare tumour characterized by its benign clinical behaviour, although local recurrence seems to occur frequently after the intervention. This neoplasm is composed of multiple small fluid-filled cysts. A 36-year-old woman, gravida 2, para 2, (two caesarean sections), with dysmenorrhea, subjected to bilateral paratubal cysts excision thirteen years ago, presented to our department. Preoperative ultrasonography showed multiple cysts in the pouch of Douglas containing a dense fluid (Fig 1). Tumor markers were within normal limits. Subsequent laparoscopy revealed a conglomerate of cystic structures $(7 \times 9 \mathrm{~cm})$ sticking to the posterofundic wall of the uterus, that spread to the pouch of Douglas (Fig 2). We removed the cysts and part of uterine serous membrane. We put a stitch on the fundic wall of the uterus. Microscopic examination demonstrated multiple cystic spaces lined by flat to cuboidal cells, and no atypia or mitoses. A diagnosis of benign multicystic mesothelioma was made. Tumor recurrence is reported in about half of the patients, even when all gross and visible tumor tissues have been removed $(1,2)$. Thus, routine follow-up imaging is required in patients after operation.

References: 1. Safioleas MC, Constantinos K, Michael S, et al. Benign multicystic peritoneal mesothelioma: a case report and review of the literature. World J Gastroenterol 2006; 12: 5739-42. 2. Sawh RN, Malpica A, Deavers MT, et al. Benign cystic mesothelioma of the peritoneum: a clinicopathologic study of 17 cases and immunohistochemical analysis of estrogen and progesterone receptor status. Hum Pathol 2003; 34: 369-74.

Key-words: cystic mesothelioma, treatment, follow-up.

\section{P1_10}

\section{Obturator nerve stimulation during transcervical resection of endometrial polyp}

M. Maher, C. Mammen

Rochdale Infirmary, United Kingdom

We present an interesting case of obturator nerve stimulation during hysteroscopic resection of an endometrial polyp. The 65 year old patient attended gynaecology outpatients department with a long history of postmenopausal bleeding. She had an early menopause aged 45 years 
and was started on hormone replacement therapy for the following 10 years. Ultrasound examination revealed a normal sized anteverted uterus with a right cornual polyp measuring $4.4 \times 5.7 \mathrm{~mm}$ in size. The patient was therefore listed for hysteroscopy and transcervical resection of polyp (TCRP). The hysteroscopy was straightforward and the right cornual polyp was identified. During resection with bipolar loop diathermy, the patient had several episodes of obturator nerve stimulation. These were represented by the patient adducting her right leg intra-operatively when the bipolar was used. The obturator nerve reflex is a well known phenomenon in urological surgery prior to the introduction of bipolar resection. This often required the need for obturator nerve block so as to minimise intraoperative complications such as bladder perforation. This practice has now been superseded by the use of bipolar resection. Electrical resistance of physiological saline and human tissues are approximately 40 and 500 omega, respectively. Therefore, the electrical current should flow within the bipolar loop and not form an electrical circuit within the patients' body. As in urological bipolar surgery, during TCRP there should not be any flow of electricity to the obturator nerve and hence stimulation. There are no other case reports in the literature with regard to intraoperative obturator nerve stimulation during TCRP. We feel that this case highlights a potential danger when resecting polyps in the cornual region, in that there is a theoretically increased risk of uterine perforation. We would therefore recommend that this increased risk be evaluated when consenting patients for TCRP and that all necessary precautions, such as an obturator nerve block, to prevent obturator nerve stimulation be considered in these cases. Key-words: Obturator nerve, polypectomy, bipolar resection.

\section{P1_11}

Total laparoscopic radical hysterectomy and bilateral pelvic lymphadenectomy for IB stage cervical cancer my first case

A. Malinowski, G. Maciolek-Blewniewska, K. Pogoda

Polish Mother's Memorial Hospital - Research Institute, Lodz, Poland, Poland

Total laparoscopic radical hysterectomy and bilateral pelvic lymphadenectomy for IB stage cervical cancer-my first case. Introduction: The clinical application of minimally invasive surgical techniques in gynaecology is constantly increasing. The laparoscopic treatment of cervical cancer is still a matter of debate and a controversial issue. Laparoscopic surgery has widely replaced open surgical technique in many routine cases. However it is widely obvious that this method is less traumatic, less painful, need shorter hospital stay and has fast recovery time. The aim of the study is to present highprecision laparoscopic method for treatment of the cervical cancer. Patient and method: A 44-years-old woman was admitted to hospital to operation because of cervical cancer stage IB1. The patient's body mass index was 23,7 . She was qualified to total laparoscopic radical hysterectomy ( Piver type III) and bilateral pelvic lymphadenectomy.

Instruments: Three five millimeter troacars and zero-degree optic laparoscopic camera was used. We also exploited other instruments like two atraumatic grasping forceps, monopolar hook, EMED ThermoStapler ${ }^{\circledR}$ device and ERBE bipolar cutting and coagulation instrument.

Results: The operating time was 220 minutes. The $\mathrm{Hb}$ drop was $1,5 \mathrm{~g} / \mathrm{dl}$. No intraoperative or postoperative complications occurred. The postoperative hospital stay was 3 days. Final histopathologic tests revealed Carcinoma planoepitheliale invasivum colli uteri with negative margins of vagina (with $5 \mathrm{~cm}$ surgical margins confirmed by histopathologic test). Parametric were intact. The tumor size was $3,5 \mathrm{~cm}$. Metastases were identified in 5 of 15 lymph nodes. Conclusions: Total radical laparoscopic hysterectomy with lymphadenectomy seems a safe and effective minimally invasive technique of treatment for IB stage cervical cancer. The laparoscopic approach may offer an alternative way for patients undergoing radical abdominal hysterectomy.

Key-words: total laparoscopic radical hysterectomy, pelvic lymphadenectomy, early stage of cervical cancer.

\section{P1_12}

\section{Silent pelvic endometriosis presenting as ureteral obstruction}

M.I. Pardo Pumar, S. Campos Arca, R. Vidal Hernández, M.E. Garcia Giménez, J.E. Moral Santamarina Complejo Hospitalario de Pontevedra, Spain

Extrinsic ureteral obstruction is a common problema in urology. The incidence of ureteral involvement in endometriosis is estimated to be around $1,2 \%$. We present a case of advanced extrinsic endometriosis in a 48 years-old woman, initially presented as obstructive pyelonephritis. Clinical symptoms were fever and right flank pain. Abdominal ultrasound examination as well as CT showed right hydronephrosis with stenosis three $\mathrm{cm}$ from the ureterovesical junction and enlarged uterus myomatosus with an inespecific right ovarían cyst $(3,5 \mathrm{~cm})$. A laparoscopy was performed jointly by a urologist and a gynaecologist. Findings were ureteral stenosis due to ovaian endometriosis. The ureter was mobilized and lifted clear of the area of endometriosis and a total laparoscopic histerectomy with bilateral adnexectomy was performed. The postoperative course was uneventful and the patient 
was discharged home on the 4 th postoperative day. The possibility of endometrisos in women with distal obstruction of the ureter should always be considered although pelvic symptoms relating to the disease are not present. Key-words: ureteral obstruction, endometriosis, hydronephrosis.

\section{P1_13}

\section{Polipectomy hysteroscopic with thermal damage of the intestinal wall and ureter - case report}

W. Pinheiro, A.K.C. Pereira, P.V. Gherpeli, D. Ejzenberg, J.M. Soares Jr., E.C. Baracat

University of São Paul, Brazil

Hysteroscopy Surgery is the gold standard for the treatment of intra uterine conditions, as endometrial polyps, fibroids sub mucosal, adhesions, etc. This type of therapy is not without complications and risk. The most common complication is uterine perforation. When drilling occurs during surgery, it is necessary that we carry out a thorough investigation of the abdominal cavity to identify thermal injury that may occur in viscera degree. The authors describe a case in which there was perforation during surgery and the lesions that were identified and treated.

Case Report: LFP, 61- year-old-woman sustained uterine perforation with resectoscope $26 \mathrm{~F}$. Perforation occurred at the time of resection of endometrial polyps with its base next to the bottom at the left uterine perforation was detected in the revision of the cavity after resection of polyp. Immediately proceeded to laparotomy to investigate the entire abdominal cavity to search for a possible injury. Were then identified two lesions, one in the intestinal wall and another that severed the left ureter, these lesions were treated properly. The patient had an uneventful postoperative period and was discharged on the eighth postoperative day.

Comments: Interestingly, in this surgical hysteroscopy have occurred the concomitant complication of ureteral section and thermal injury of the intestinal wall. Not found in the literature so far any reference to ureteral injury in surgical hysteroscopy, hence we believe this to be the first report worldwide.

Key-words: complications in hysteroscopy, ureteral injury, uterine perforation.

\section{P1_14}

Acute renal failure after surgery for hysteroscopic polypectomy - case report

W. Pinheiro, A.K.C. Pereira, P.V. Gherpeli, D. Ejzenberg, E.C. Baracat

University of São Paulo, Brazil
Introduction:Hysteroscopy is a minimally invasive procedure having many benefits compared with traditional procedures. Potential risk includes fluid overload, uterine perforation, technical problems, hemorrhage, infection The authors present a case of acute renal failure in patients operated for endometrial polyp by hysteroscopy.

Case report:We present a 51 year-old, Asian woman, who underwent polypectomy hysteroscopy under general anaesthesia.The distention's minds mannitol 3\% $1500 \mathrm{ml}$. In the postoperative she presents oliguria $200 \mathrm{ml} / \mathrm{d}$, headaches, nausea and vomiting. The serum electrolytes showed: Sodium $124 \mathrm{mEq} / \mathrm{L}$, Potassium 4,8 Phosphorus 7,1,C-Reactive Protein17,7 mg/l,Urea 131, Creatinine 6,46, Lactate Dehydrogenase $875 \mathrm{U} / \mathrm{L}$, Magnesium2,24 mg/dLIonized Calcium 3,0 mg/dL, Chlorine $97 \mathrm{mEq} / \mathrm{L}$,Calcium 7,9 mg/dL.The $\mathrm{Ct}$ of the kidneys indicated that it was renal parenchymal injury stage 3. Analysis of urine revealed: proteinuria $0,3 \mathrm{~g} /$ 1 and granular and hyaline casts. The patient was treated with Saline $1500 \mathrm{ml} / \mathrm{d}$. She was treated with physiological serum solution $1500 \mathrm{ml} / \mathrm{d}$. Evolution: after five days of treatment the chemist serum showed; Calcium 9,5,Chlorine 105, Urea 25, Creatinine 0,55, Potassium 3,8, Magnesium 2,02, Sodium 140, Phosphorus 3,5.We concluded that the dilution hyponatremia in hysteroscopic polypectomy is rare, apart from the fact that we used only $1500 \mathrm{ml}$ of mannitol, thus being in agreement with those who think that acute renal failure in these cases is multifactorial.

Key-words: hysteroscopy, renal failure, hyponatremia.

\section{P1_15}

\section{Transmigration of intrauterine devices \\ M. Rodrigues, A. Maçães, F. Geraldes \\ Bissaya Barreto Maternity, Portugal}

The intrauterine device (IUD) is one of the most popular reversible contraception method used worlwide. One of the serious, but uncommon, complications of IUD is perforation through the uterine wall into pelvic or abdominal cavity. Migrant IUDs may remain asymptomatic for years or may cause serious complications. Transmigration of IUD consequently leading to bladder perforation, which is a rare event with few cases described in literature, or bowel perforation. The authors report 2 cases of complete migration of IUD to abdomino-pelvic cavity. First case was a forty yearsold woman who underwent a tubal ligation by laparoscopy, and during the procedure, they saw what seemed to be wires lying on left parieto-colic adhesion, despite 
the patient had no surgical history. She had a history of device insertion three years before, and was presumed to have been expulsed spontaneously. Abdomino-pelvic $\mathrm{X}$-ray was performed, demonstrating an IUD located outside the uterus, close to the pubic symphysis on the left side. Pelvic magnetic resonance imaging showed IUD in pelvic cavity predominantly intra-vesical. Endoscopic intervention was planned by Urology department, and IUD was removed by transurethral approach without any complications. The postoperative course was uneventful. The second case was a fifty-one yearsold woman in whom a levonorgestrel-releasing intrauterine system had been placed three years previously, and the strings were not observed in routine gynaecologic examination. Ultrasonography revealed an empty uterus. Abdominal X-ray and MRI showed the IUD next to the cecum. Laparoscopic was performed and IUD was removed. The authors conclude that endoscopic methods are efficient and safe for the treatment of such cases.

Key-words: mislocated IUD, uterine perforation, laparoscopy.

\section{P1_16}

\section{Embolization of the uterine arteries after total laparoscopic hysterectomy: a case report}

D. van de Burgt, A.L. Aalders, F.P.H.L.J. Dijkhuizen

Department of Obstetrics and Gynaecology, Rijnstate Hospital Arnhem, The Netherlands

A 47-year old woman attended our outpatient clinic with complaints of backpain and abnormal uterine bleeding. Transvaginal ultrasonography showed a intramural leiomyoma in the anterior wall of the uterus measuring $4 \mathrm{~cm}$ in diameter. Intracavitary abnormalities were ruled out. Medical treatment, including a progesterone intrauterine device, did not solve the problem. Therefore, a total laparoscopic hysterectomy was performed. This was carried out without any complications and with a total bloodloss of $150 \mathrm{ml}$. Postoperative recovery also occurred without any complications. Fourteen days after surgery the patient was brought to the hospital with heavy vaginal bloodloss. The bleeding was due to bloodloss from the left uterine artery. We were not able to control the bleeding by suturing vaginally. To avoid laparotomy, a uterine artery embolisation was performed. This appeared to be succesfull and the bleeding stopped. After bloodtransfusion the patient recovered quickly and she was discharged from the hospital 3 days after surgery.

Key-words: Uterine embolisation, laparoscopic hysterectomy, complications.

\section{P1 17}

Hysteroscopical removal of a Bakri-balloon: a case report

D. van de Burgt, A. Huisman, F.P.H.L.J. Dijkhuizen

Department of Obstetrics and Gynaecology, Rijnstate Hospital Arnhem, The Netherlands

A 28-year old nulliparous patient underwent an elective caesarean section because of breech presentation. During pregnancy she had been diagnosed with a leiomyoma in the anterior wall of the uterus measuring $55 \times 40 \mathrm{~mm}$. During the caesarean section a healthy daughter was born of 3400 grams when extensive blood loss occurred due to uterine atonia. Administering uterotonic agents (Oxytocin and Sulprostone) did not stop the bleeding. A Bakri-balloon was inserted in the uterine cavity abdominally and filled with saline. At the same time, embolisation of the uterine arteries was performed by the radiologist. After the two procedures the massive bleeding stopped. The total amount of bloodloss was more than $4000 \mathrm{ml}$. Twelve hours after the operation we attempted to remove the Bakri-balloon but this appeared to be unsuccessful. Strikingly, we were not able to remove any saline from the balloon. Abdominal ultrasound showed that the balloon appeared to be empty. We presumed that the balloon had been stitched to the uterine wall during closure of the uterus. A hysteroscopy was then performed in order to prevent our patient from relaparotomy. Fortunately, we were able to identify a single stitch that had been pierced through the outer lumen of the Bakri-balloon's hose. After cutting this stitch hysteroscopically, we could remove the balloon easily. The uterine wall was inspected after removal of the balloon and appeared to be intact. No further blood loss occurred and the patient was able to recover quickly. She was discharged from the hospital 5 days after surgery.

Key-words: therapeutic hysteroscopy, Bakri-balloon, operative hysteroscopy

\section{P1_18}

Before pregnancy laparoscopic cervical cerclage using cerclage tape. Two case reports and literature review

E. Xia, Y. Liu, X. Huang, J. Zheng, D. Yu, Y. Li, J. Qu Hysteroscopic Center, Fuxing Hospital, Capital Medical University, China

Summary: Cervical cerclage is an effective treatment method of cervical incompetence, vaginal or abdominal treatments will have a higher success rate, about $81 \%-87 \%$. In the past time n.10 silk or nylon string were used to suture 
and tie the cervix. Since the polypropylene tape comes out, application of cervical cerclage tape reported increased gradually.

Introduction: The safety and effectiveness of abdominal or laparoscopic cervical cerclage using cerclage tape was evaluated.

Materials and Methods: Abdominal or laparoscopic cervical cerclage using cerclage tape suture was placed before pregnancy to treat two women who had history of late abortion. Tension knots of cerclage were tied at the level of cervical inner os.

Results: Case 1- $35 \mathrm{~W}+6$ gestation pregnancy, delivered a girl by cesarean section with 2900 gm weight at March 2, 2009. Central previa placenta. Case 2- HSG showed both fallopian tubes blocked at December 2009. Twice fluid cannulation was performed and failed.

Discussion: Transvaginal cervical cerclage tying the inner os at the level of inner os was often fail and caused vaginal infections. Transabdominal cervical cerclage is quite appropriate for those women who had failure history of vaginal cerclage and $95 \%$ can get live baby. Laparoscopic cervical cerclage with polypropylene tape was minimally invasive technique which had the same outcome of abdominal cerclage.

Key-words: cervical incompetence, cervical cerclage tape, laparoscopy.

\section{P1_19}

\section{Case report: an adenomatoid tumour associated with interstitial ectopic pregnancy and techniques for laparoscopic excision \\ E. Craig, J. Beirne, K. Johnston \\ Antrim Area Hospital, Department of Gynaecology, North- ern Ireland}

Introduction: A case report that describes a rare aetiology and the feasibility of laparoscopically managing large cornual pregnancies. Cornual pregnancy is a very uncommon site for ectopic implantation (overall incidence $2 \%$ ), usually presenting with uterine rupture, a compromised patient and managed with open resection. We present a large unruptured cornual pregnancy managed laparoscopically, associated with an adenomatoid tumour - a very uncommon, benign, mesothelial tumour. Only one similar associated case is reported in the literature.

Materials and Methods: A case report of a 41 year old para 3. Initial presentation was at 11 weeks amenorrhoea with vaginal staining. Abdominal and pelvic examination elicited no tenderness. Transvaginal ultrasound demonstrated a $3 \times 4 \mathrm{~cm}$ right mixed echogenic adnexal mass, sited at the uterine cornua, no free fluid seen. The patient underwent a laparoscopic resection of this ectopic gestation. A $3 \times 4 \mathrm{~cm}$ vascular cornual ectopic was excised laparoscopically. The base was initially injected with $1 \%$ Xylocaine and 1:200,000 Adrenaline to vasoconstrict surrounding vessels. An ENDOLOOP ${ }^{\circledR}$ Ligature PDS II suture was used to develop a pedicle and secure the base of the ectopic. Bipolar diathermy was also applied to the base. Three intracorporeal $1 / 0$ Vicryl ${ }^{\circledR}$ sutures further secured haemostasis and facilitated uterine repair.

Results: Estimated blood loss was $100 \mathrm{mls}$. The patient was discharged within 24 hours. The adenomatoid tumour, was an incidental finding, but has been implicated in the pathogenesis due to distortion of the local tubal interstitial architecture.

Discussion: With appropriate surgical expertise, laparoscopic management of even large cornual ectopic pregnancies is feasible in haemodynamically stable patients. Adenomatoid tumours can be an extremely rare underlying aetiology in cornual pregnancy

Key-words: Adenomatoid tumour, interstitial ectopic.

\section{P1_20}

\section{Endometrial ablation and the complications of post procedure pregnancy: case reports and review of literature}

B. Smyth, E. Craig, G. Norris, W.A. Ritchie, R. McMillan, G.J. Dorman

Antrim Area Hospital, Northern Ireland

Introduction: Endometrial ablation is a commonly used treatment for menorrhagia. Following endometrial ablation pregnancy is an absolute contraindication given the associated risks including early and late pregnancy loss, haemorrhage, morbidly adherent placentation, malpresentation and increased perinatal mortality. It is essential to ensure that the most reliable method of contraception is in place.

Materials and methods: A retrospective review of two case reports.

Results: We present 2 cases of pregnancy after endometrial ablation. The first case led to a mid trimester loss with adherent placenta and significant blood loss. The second case, conceived on POP resulted in a term pregnancy delivered by caesarean section for breech presentation with placenta increta and PPH.

Discussion: Due to poor patient compliance and contraception failure rates pregnancy after endometrial ablation may still occur. We demonstrate the importance of counselling patients pre and post endometrial ablation thoroughly regarding the risks and complications, as illustrated by these cases. Key-words: Endometrial ablation, complications, pregnancy. 


\section{P1 21}

\section{Laparoendoscopic Single-Site Surgery (LESS). Presentation of our experience}

Y. Cabrera, B. Bueno, S. Iniesta, C. García, P. Lobo, J. Alvarez

Hospital Infanta Sofia, Madrid, Spain

Introduction: The NOTES concept refers to any surgical procedure that uses one or more natural orifices of the body of the patient (mouth, nose, anus, vagina, urethra) in order to penetrate these hollow organs and enter another inaccessible cavity of the body. The term LESS is used for those minimally invasive procedures performed through a single abdominal incision, generally umbilical, using one or more laparoscopic ports or through one or several small incisions at the same site. It includes terms like TUE (transumbilical endoscopic surgery), NOTUS (natural orifice transumbilical surgery), SPL/SPA (single port laparoscopy/single port Access), e-NOTES (embryonic NOTICE), U-NOTES (umbilical natural orifice translumenal endoscopic surgery), OPUS (one port umbilical surgery), SILS (single incision laparoscopic surgery), etc. We present our initial experience in LESS using umbilical multi-channel port for the treatment of benign adnexal pathologies.

Materials and methods: Presentation of our experience and literature review.

Results: Our data are similar to those described by other authors (with larger series) of women undergoing benign adnexal surgery. Mean adnexial cyst size was $6 \mathrm{~cm}$. All procedures were performed by the same surgeon. The average surgical time was 1 hour. Conversion to a multiaccess standard laparoscopic technique was not required in any patient and no intraoperative complications were observed. All patients were discharged from the hospital after 24 hours. Case 1: Diagnossi:Paraadnexial cyst $5 \mathrm{~cm}$ Technique:Left cystectomy. Age: 22. BMI 21,5. Time:60 min. Hospital stay: 1 day. Case 2: Diagnosis: Adnexial cyst $7 \mathrm{~cm}$. Technique:Bilateral adnexectomy. Age:58 BMI 22,3. Time:75 min. Hospital stay: 1 day. Case 3: Diagnosis: Adnexial cyst $6 \mathrm{~cm}$. Technique: Bilateral adnexectomy. Age:57 BMI 30 Time: 45 min. Hospital stay: 1 day.

Discussion: The operation using a unique port is modified laparoscopic technique that allows a minimally invasive surgery with excellent cosmetic results reducing the potential morbidity associated with several cutaneous incisions, like epigastric vessels injuries or postoperative hernias at entrance ports. Furthermore, the pneumoperitoneum insufflation under direct vision lowers the risk of injuries of abdominal organs and large vessels. Postoperative pain is reduced because abdominal muscles are not affected so analgesics consumption, hospital stay and convalescence are reduced. LESS allows an easier cyst enucleation or salpingo-oophorectomy and a more comfortable fascial suture. Also, at any time it is possible to change to a conventional laparoscopic. Despite the advantages there are significant drawbacks: unavoidable learning curve, loss of the triangulation and difficult instrument and surgeon movements that affects ergonomy and increases surgical time. Some disadvantages can be solved with the use of specific instruments such as: flexible videolaparoscopic for a better visualization of the cavity peritoneal and the use of rigid curved instruments to avoid crossing between the instruments and the camera, and to permit lateral movement into the abdominal cavity. In our experience, LESS represents a promising, feasible, safe, effective technique, with fast learning curve and good cosmetic results. Moreover, it is associated with short hospital stay and less postoperative pain. We believe that standardizing the techniques and using specific instruments will further decrease operative time, increase ergonomy, and expand the range of indications for its use.

Key-words: Adnexal pathologies, laparoendoscopic single site surgery, single port access.

\section{P1 22}

\section{Laparoscopic abdominal cerclage placement} during pregnancy. Clinical case and revision

Y. Cabrera, J. Alvarez, P. Rubio, S. Gámir, P. Lobo, C. García.

Hospital Infanta Sofia, Madrid, Spain

Introduction: Cervical incompetence is defined as structural weakness of the cervix leading to inability of the cervix to sustain an intrauterine pregnancy. It causes $8 \%$ of the losses of the second trimester. The etiology is multifactorial but in most cases it is the result of previous obstetric or gynecologic trauma like cervical conisation. The traditional treatment is the cervical cerclage described by Shirodkar (1955) or McDonald (1957). In 1965 Benson and Durfee described the first abdominal cerclage and was Scibeta (1997) and Lesser (1998) that presented the first cases of laparoscopic transabdominal cerclage (LTCC) before and during pregnancy respectively. Since then, several reports appeared in the literature with excellent results and few complications. We described our first LTCC case during pregnancy in a patient with radical trachelectomy.

Materials and Methods: Presentation of a case report and systematic revision of literature.

Results: A 40-year-old, gravida 1, was refered to our hospital with unique clomifene induced pregnancy. Her past medical history included a radical trachelectomy caused by 
early-stage adenocervical cancer (IB) with normal later revisions. Transvaginal ultrasound confirmed a singleton intrauterine pregnancy without markers of chromosomal defect. Her cervical length was short with a functional length of $1.3 \mathrm{~cm}$. On physical examination, the cervix was absence. The patient was informed that laparoscopic transabdominal cerclage placement had not been previously performed in our hospital. After informed consent was obtained, she was operated on the 14th week of pregnancy. Following induction of general anesthesia, the patient was placed in dorsolithotomy position, and a Foley catheter was inserted into de urinary bladder. A Veress needle was inserted through the Palmer point and carbon dioxide was insufflated into the peritoneal cavity for pneumoperitoneum to a $15 \mathrm{~mm} \mathrm{Hg}$ of intrabdominal pressure. The laparoscope was introduced through a $10-\mathrm{mm}$ trocar using an umbilical incision. Three 5-mm trocars were placed through the suprapubic area and in each lower quadrant laterally. An intraabdominal pressure of $12 \mathrm{~mm} \mathrm{Hg}$ was maintained. The patient was placed in steep Trendelenburg position. A member of the surgical team placed a gloved hand into the vagina intermittently to manipulate the uterus. The uterovesical peritoneal reflection was divided transversely with laparoscopic scissors and bladder was carefully reflected downward to expose the uterine isthmus and uterine vessels. Each end of the tape (Mersilene 5-mm suture) was inserted lateral to the uterus and medial to the uterine vessels at the level of the uterine isthmus above the uterosacral ligament and were tied together anteriorly by intracorporeal knot tying. The procedure lasted 263 minutes without complications and estimated blood loss was $100 \mathrm{ml}$. Fetal heart tones were confirmed before and after the procedure and the patient was discharged from the hospital three days later. The rest of the gestation attended with normality. A low transverse caesarean section was taken after preterm premature rupture of membranes at 36.4 weeks. The infant weighed 2780 gr and Apgar test was 9/10. Due to a uterine atony resistant to treatment an obstetrical total hysterectomy was performed. Mother and baby were discharged from the hospital after six days without more complications.

Discussion: Pregnancy after trachelectomy is associated with several adverse pregnancy outcomes including second trimester abortion and preterm delivery. In the absence of the portio vaginalis (for example, after a radical trachelectomy), a transabdominal cerclage may be the only option. In expert hands there are no differences in fetal survival rate based on the moment (before versus during pregnancy) or in the route (laparotomy versus laparoscopic abdominal cerclage). The LTCC is an exceptional procedure with elevated rate of successes and certain advantages like smaller blood loss, less postoperative pain, reduced risk of adhesions and shorter hospital stay compared to the laparotomy route. Due to its difficulty and possible complications (hemorrhage, fetal loss, uterine rupture, infection, rectovaginal migration of the suture and fistulas) it is reserved for extremely short cervix or failure of previous vaginal cerclage.

Key-words: cerclage, abdominal, laparoscopic.

\section{P1 23}

\section{Laparoscopic cornuostomy - Treatment of nonruptured cornual pregnancies: A case report \\ I. Chatzipapas, A. Protopapas, A. Liapi, S. Athanasiou, A. Antsaklis \\ $1^{\text {st }}$ University Department of Obstetrics and Gynaecology, Alexandra Hospital, Athens, Greece}

Objective: We describe a novel laparoscopic technique of terminating cornual pregnancies. Introduction: The incidence of ectopic gestation is approximately 20 per 1000 pregnancies. Cornual pregnancies account for 3\% of ectopic gestations. The mortality rate for a woman diagnosed with such a pregnancy is $2 \%$ to $2.5 \%$. Is one of the most hazardous types of ectopic gestation with high mortality. Different modalities of surgical intervention for cornual gestation were reported in the literature . Laparotomy with cornual resection was traditionally performed in ruptured corunal pregnancy when the patient is haemodynamically unstable. Cornual resection along with salpingectomy has been the treatment of choice in patients with no concern for future fertility. Laparoscopic and ultrasound guided transcervical evacuation of cornual ectopic pregnancy have also been described. However, conservative surgical or medical approaches are preferred in subfertile patients.

Procedure: Laparoscopic findings established a diagnosis of unruptured cornual pregnancy. Initial hemostasis is achieved with dilute vasopressin inject throughout the myometrial capsule, then a linear incision was made along the long axis of the ectopic mass using monopolar needle. The ectopic sac was pulled gently with a grasping forceps through the incision, then trophoblastic material was drainaged carefully with a negative pressure suction aspiration device and spoon forceps. The bleeding area of the myometrium was coagulated using bipolar diathermy forceps. The uterine incision was closed using interrupted suture Vicryl 1. The resected cornual pregnancy was then removed with an Endobag. Conclusion: Laparoscopy has become the gold standard treatment for cornual pregnancies in the last few years and generally has replaced laparotomic procedures.

Key-words: laparoscopic, cornuostomy. 


\section{P1 24}

\section{Hysteroscopy in the diagnosis of colouterine fistula: a rare complication of diverticulitis}

C. Nogueira-Silva, L. Castro, F. Rodrigues, H. Marques, P. Leão, A. Rocha

Department of Obstetrics and Gynaecology and Department of General Surgery, Hospital of Braga, Portugal

Summary: Colouterine fistula of diverticular etiology is an extremely rare complication. Ideal methods for diagnosis remain to be established. The authors report one case of sigmoid-uterine fistula in which hysteroscopy was essential for the diagnosis confirmation.

Introduction: Colouterine fistula, secondary to diverticulitis, is an extremely rare disease (only few cases have been reported in the literature) due to the resistance of uterine tissue.

Case report: A 76 year-old woman reported faecal vaginal discharge over a 6-month period. Several gynaecological examinations did not confirm vaginal faeces. Computed tomography revealed a possible undefined recto-vaginal fistule, but colonoscopy was normal. Magnetic resonance (MR) suspected a fistula between the anterior aspect of the uterus and the sigmoid colon which contained extensive diverticulosis. Hysteroscopy confirmed the fistulous opening of uterus. Surgical treatment consisted of sigmoid colectomy and hysterorraphy, followed by an uneventful recovery.

Discussion: This case report demonstrates that MR may help to establish a proper diagnosis, but confirmation of colouterine fistula may be reached by diagnostic hysteroscopy. The sensitivity and specificity of this procedure to diagnose such cases remain to be established.

Key-words: colouterine fistula, hysteroscopy.

\section{P1_25}

GIST (Gastrointestinal Stromal Tumors): as a differential diagnosis of adnexal tumors

S. Iniesta, P. Lobo, S. Gámir, Y. Cabrera, B. Bueno, J. Álvarez

Hospital Infanta Sofia, San Sebastian de Los Reyes, Madrid, Spain

Introduction: GIST are the most frequent variety of mesenchymal tumours of the digestive tract, but they hardly represent 1 to $3 \%$ of the malignant gastrointestinal neoplasms. Their estimated prevalence is $15-20$ cases per million (the incidence of GIST in Spain is 457 new cases per year) and it affect to patients at sixty-year old median age. Half of the cases occur in the stomach; this half may also be confined to an specific organ, like small intestine $(30 \%)$, rectum (5\%) and colon (2\%); Occasionally the primary GIST may occur in the peritoneum, pancreas, ovaries, uterus or prostate. GIST main symptom may be either a painful mass or unspecific symptomatology; nonetheless, bleeding and obstruction must be considered as well. The diagnosis must be histopathologic and its confirmation immunohistochemical: $95 \%$ of GIST reacts positively to KIT or CD117. The malignant behaviour of the tumour is determined by its size and the mitosis images.

Material and methods: We present a case of GIST, that was proposed for an intraoperative biopsy after a diagnosis of an adnexal tumor with suspicious of malignancy, with both markers and imaging tests.

Results: Woman of 60 years old, from Colombia, who complains us because a clinical revision, completly asympthomatic. She had a previous gynaecological control 2 years before, without anything remarkable. Nulligravida with menopause at 45 years old. - Gynaecological exploration: Increased size of the left adnexal area. - Gynaecological ultrasound: increased size of the left ovary, of $67 \times$ $42 \mathrm{~mm}$, with an irregular contour, with inside areas of mixed echogenicity, hyperechogenicity (with shadowing) and with papillary formations inside the echonegative ones; abundant Doppler color signal inside the tumor, with predominantly venous morphology (IR 0.6). Both uterus and left ovary were described as normal. There was no pelvic liquid during the examination. CT abdpelvic: In small pelvis, adjacent to the uterine fundus, displayed two ovoid lesions of 3.5 and $4.2 \mathrm{~cm}$ of heterogeneity behavior, with big calcifications, presenting the one of smaller size some hypoenhancing areas. Because of the morphological appearance they could correspond to subserosal fibroids. Ovaries are not displayed. Intestinal loops with distribution and morphological apparently normal. Multiple intrahepatic LOES, radiologically indeterminate. Rest of the study without any alteration. - Tumor markers: Both CEA and CA 125 were normal. CA 19.9 was high: $635.8 \mathrm{U} / \mathrm{ml}$. Laparoscopy: we visualized a lobulated, hard and highly vascularized mass that was pedunculated in an intestinal loop, located at the pelvis, of $5 \mathrm{~cm}$ of diameter, very close to the left adnexal area. Uterus and both ovaries were macroscopically normal.

Discussion: In the differential diagnosis of adnexal tumors we must consider the possible non gynaecological origin, including those of intestinal origin. A very specially preoperative evaluation must be performed before the surgery in order to explain to our patient the suspected pathology, the posible surgery and the postoperative cares.

Key-words: GIST, CA 19.9, differential diagnosis adnexal tumors. 
P1 26

Hysteroscopic treatment of obstructed hemivagina with uterus didelphys with ipsilateral renal agenesis: a case report

T. Var, A. Ozturk, E. Tonguc, S. Batioglu

Zekai Tahir Burak Women Health Education and Resarch Hospital, Turkey

Introduction: Uterus didelphys with obstructed hemivagina and ipsilateral renal agenesis is an anomaly seen rarely. Excision of the obstructed vaginal septum is the main treatment.Hysteroscopic resection of the vaginal septum can be used instead of conventional excision treatment especially in virgin patients.

Case report: 13 years old female patient without complaint was sent to an internal diseases clinics for advanced examination since she was detected to be $\mathrm{Hbs} \mathrm{Ag}(+)$ during hepatitis screening in the school. A mass was detected on the left renal agenesia and pelvic area as result of abdomino-pelvic ultrasonography and the patient was directed to Zekai Tahir Burak Women's Health Education and research Hospital Adolescent Polyclinics. The patient has been menstruating irregularly (oligo-menorrheic) for 1 year. The patient had dysmenorrhea and were defining her menstrual as regular. Physical examinations made on the patient gave normal results. No genital examination could be made since the patient was virgin. However, a soft-tissue mass was found in the left vaginal localization in her rectal examination. A cystic mass containing $90 \times$ $40 \mathrm{~mm}$ sized and low level internal echoes located on the left vagina was found in the pelvic and abdominal ultrasonography taken by our hospital's radiology unit. In addition, two widely divergent uterine horns with no communication were detected. Left kidney could not be observed. Since vaginal examination could not be made the laparoscopy and vaginoscopy were planned in order to confirm the diagnosis. Uterus was observed didelphically in laparoscopic examination. Two tuba-uterine and ovary relations were observed normally. The vagina was entered through introitus because of social structure of our country. Right cervical ostium was observed. It was observed that the vagina was pushed from left side. Longitudinal septum was cut by the means of electrocautery. Hematoma was emptied, septum resection was completed and the vagina was enlarged. Left and right cervixes were observed .

Conclusion: Hysteroscopic resection of the vaginal septum is excellent and efective alternative to conventional exicion treatment in patients of Uterus didelphys with obstructed hemivagina and ipsilateral renal agenesis

Key-words: hysteroscopy, obstructed hemivagina with uterus didelphys with ipsilateral renal agenesis.
Poster Session 2_Complications

\section{P2 1}

Transurethral removal of eroded tension free vaginal tape

A. Thomas, S. George, H. Hamed

St Helens \& Knowsley Teaching Hospitals NHS Trust; Stockport NHS Foundation Trust, UK

Introduction: Tension free vaginal tape (TVT) is a widely accepted treatment of choice for women with stress urinary incontinence. It is a minimally invasive procedure with short procedure times and post operative stays with low complication rates.

Case report: We describe a case of delayed urethral erosion of a TVT sling 6 years after insertion who presented with vaginal discomfort and recurrent incontinence.The tape was removed under cystoscopic control without incising urethra or vagina. Post operatively patient reported complete resolution of symptoms.

Discussion: Tape erosion is an uncommon complication which is usually noticed into the vagina.Urethral erosion is unusual and a delayed presentation such as this has not been previously reported. It is challenging to manage and is associated with significant morbidity as urethral incision is usually necessary. In this case urethral incision was avoided, with very good results.

Key-words: TVT, delayed erosion, transurethral.

\section{Poster Session 3_Endometriosis: Diagnosis}

\section{P3_1}

Voiding dysfunction in patients with severe deep infiltrating endometriosis: assessment before and after laparoscopic treatment

M. Martínez-Terrón, M.A. Martínez-Zamora, F. Carmona, M. Espuña, M. Puig-Clota, A. Franco, J. Balasch Institut Clínic de Ginecologia, Obstetrícia i Neonatologia. Institut Clínic de Nefrologia i Urologia. Hospital Clínic de Barcelona. University of Barcelona, Spain

Summary: Voiding dysfunction (VD) is a frequent complication of the laparoscopic treatment of severe deep infiltrating endometriosis (DIE). The preoperative urodynamic testing showed a high incidence of bladder dysfunctions.

Introduction: Autonomic nerve damage plays a crucial role in the aetiology of bladder dysfunction that occurs after 
resection of DIE. We assessed the association of bladder dysfunction and the laparoscopic surgery of severe DIE.

Methods: Urodynamic testings were performed before surgery and 4 months after surgery. Those patients who complained of incomplete emptying, strain to start or reduced stream after surgery were tested with a noninvasive flowmetry, before performing the urodynamic testing. Severe DIE was considered if there was bowel infiltration and/or $>2$ lesions of histologically diagnosed DIE and/or $>1$ of the endometriotic nodules measured $\geq 4 \mathrm{~cm}$. Patients with ureteral or bladder involvement were excluded.

Results: Thirty-five patients were analyzed. All patients underwent complete laparoscopic resection of DIE. Four patients complained of urinary symptoms before surgery. The preoperative urodynamic studies showed VD in 8 patients. The postoperative urdynamic testing showed VD in 15 patients, although only 7 patients complained about urinary symptoms. Two patients have severe VD and require intermittent self-catheterisation one year after surgery. Both patients had pathologic preoperative urodynamic testings.

Discussion: VD is a frequent complication of the laparoscopic treatment of severe DIE and patients should be informed about this possible complication before surgery. Nevertheless, the preoperative urodynamic testing showed a high incidence of bladder dysfunctions and may be useful to predict severe VD after surgery.

Key-words: voiding disfunction, deep infiltrating endometriosis, urodynamic testings.

\section{P3_2}

Ovarian endometriosis and its association with severe deep infiltrating endometriosis

A. Borrás, M.A. Martínez-Zamora, F. Carmona, R. Rull, $\overline{\text { J. Balasch }}$

Institut Clínic de Ginecologia, Obstetrícia i Neonatologia. Institut Clínic de Malalties Metabòliques i Digestives. Hospital Clinic de Barcelona. University of Barcelona, Spain

Summary: Ovarian endometriomas, either unilateral or bilateral, are frequently associated with severe DIE.

Introduction: Ovarian endometriomas can easily be treated laparoscopically. However, deep infiltrating endometriosis (DIE) need to be treated in specialist endometriosis centers used to deal with laparoscopic advanced techniques. We investigated the association of ovarian endometriosis and severe DIE lesions treated by laparoscopy.
Methods: One hundred eleven patients histologically diagnosed of DIE and laparoscopically treated were analyzed. All implants of endometriosis were removed during surgery and fertility was conserved in all patients. Severe deep infiltrating endometriosis was considered if there was bowel and/or ureteral and/or vesical infiltration and/or if there were more than two lesions of histologically diagnosed DIE and/or if at least one of the endometriotic nodules measured $\geq 4 \mathrm{~cm}$.

Results: Mean age of patients was $32.7 \pm 5.4$ years. Fiftyseven patients had endometriomas $(51.3 \%)$. Twenty-nine $(26.1 \%)$ were unilateral and twenty-eight (25.2\%) were bilateral. Mean size of the biggest endometrioma: $43.6 \pm 24 \mathrm{~mm}$. Mean size of the sum of all endometriomas: $74.1 \pm 33 \mathrm{~mm}$. Sixteen patients $(14.4 \%)$ did not have severe DIE or endometriomas. Thirthy-eight patients $(34.2 \%)$ had severe DIE but did not have endometriomas. Patients having unilateral ovarian endometriomas $(n=29)$ had severe DIE in twenty-six cases $(89.6 \%) \quad(p=0.002)$. Patients having bilateral ovarian endometriomas $(\mathrm{n}=28)$ had severe DIE in all cases $(100 \%)(\mathrm{p}=0.002)$.

Discussion: Ovarian endometriomas, either unilateral or bilateral, are frequently associated with severe DIE. Thus, the diagnosis of ovarian endometriomas before surgery should aware gynaecologists to properly diagnose and treat associated severe DIE.

Key-words: ovarian endometriosis, deep infiltrating endometriosis, endometrioma.

\section{P3_3}

Diagnostic accuracy of intravenous pielography and magnetic resonance imaging to diagnose ureteral endometriosis: correlation with laparoscopy

I. González, M.A. Martínez-Zamora, S. Anglès, L. Buñesch, F. Carmona, A. Franco, J. Balasch

Institut Clínic de Ginecologia, Obstetrícia i Neonatologia. Institut Clínic de Nefrologia i Urologia. Institut de radiologia. Hospital Clínic of Barcelona. University of Barcelona, Spain

Summary: The combination of intravenous pielography (IP) and magnetic resonance imaging (MRI) showed high accuraty in prediction of severe infiltrating UE.

Introduction: UE is an uncommon site of deep infiltrating endometriosis (DIE). A presurgical diagnosis of severe UE is essential to perform an efficient laparoscopic surgery. We evaluated the accuracy of IP and MRI in the diagnosis of severe UE.

Methods: Retrospective analysis of 112 patients with histological proved DIE. The preoperative findings with IP and MRI were evaluated after surgery. 
Results: 29 patients had histological proved UE (16 left, 8 right and 5 bilateral). In all patients, UE was laparoscopically confirmed and complete surgical excision was performed. Surgical and histological results showed severe infiltrating UE in 18 patients $(62 \%)$. IP detected UE in $89 \%(n=16)$ of those patients with severe infiltrating UE. The sensitivity (S) and specificity (E) of IP for the diagnosis of severe infiltrating UE were $90 \%$ and $100 \%$. MRI detected severe UE in $72 \%$ of patients $(n=13)$. The $S$ and $E$ of MRI for the diagnosis of severe infiltrating UE were $78 \%$ and $100 \%$. In one case of severe infiltrating UE without hydroureteronephrosis both, IP and MRI, did not diagnose the UE. The combination of IP and MRI increases the $\mathrm{S}$ for the diagnosis of severe infiltrating UE to $95 \%$. Ten patients $(34.5 \%)$ presented with severe UE which caused moderate to severe hydroureteronephrosis (7 left, 2 right and 1 bilateral). All cases of hydroutereronephrosis were presurgically diagnosed by IP and MRI.

Discussion: MRI and IP allow the diagnosis of severe UE in all cases in which hydroureteronephrosis is associated. The combination of IP and MRI demonstrated high accuraty in prediction of severe infiltrating UE.

Key-words: ureteral endometriosis, intravenous pielography, magnetic resonance imaging.

\section{P3_4}

\section{Alexithymia in patients with endometriosis and chronic pelvic pain}

I. Melis, S. Angioni, I. Arena, S. Pirarba, E. Stochino Loi, M. Dessole, G.B. Melis

Division of Gynaecology, Obstetrics and Pathophysiology of Human Reproduction, University of Cagliari, Italy

Introduction: Alexithymia appears to affect the subject's ability to deal with the diversity of subjective pain factors although the mechanism through which it influences the perception of pain remains to be clarified. Aim of the study was to evaluate the presence of the alexithymic trait in a group of patients with endometriosis and chronic pelvic pain in comparison to healthy controls using a validated tool.

Methods: 41 patients with pain and surgical diagnosis of endometriosis and 40 healthy controls were recruited in the study. All subjects taking part in the study underwent a semi-structured interview by a psychologist and the Italian version of the Toronto Alexithymia Scale (Tas-20), used to assess the presence of alexithymia was administered. Following interview the physician evaluated the intensity of chronic pelvic pain in the patients by means of a modified version of the Biberoglu pain scale.
Results: A positive score for alexithymia was achieved by $14.6 \%$ (n. 6) of patients with, whilst this condition was absent in the control group; alexithymia was indeterminate in $29.3 \%$ (n. 12) and $12.5 \%$ (n. 5) of patients and controls, respectively, and absent in $56.1 \%$ (n. 2) and $87.5 \%$ (n. 35). Inter-group differences were significant for three factors evaluated by TAS-20: difficulty in identifying feelings and distinguishing from bodily sensations (Fact. 1), difficulty in describing feelings (Fact. 2), and externally-oriented, or operative thinking (Fact. 3).

Discussion: The present study underlined how women with endometriosis were significantly more alexithymic compared to controls. Effective treatment of chronic pelvic pain should therefore be based not only on an accurate medical assessment, but also on a knowledge of the emotional management of the disease and its psychological consequences. (Founding: Regione Autonoma della Sardegna, Programma Master and Back. Beneficiaria: Dott.ssa Irene Melis, Psicologa)

Key-words: endometriosi, chronic pain, alexithymia.

\section{Poster Session 4_Endometriosis: Surgery}

\section{P4_1}

94 months follow up after laparoscopic-assisted vaginal resection of septum rectovaginale and rectosigmoid in women with deep infiltrating endometriosis

A. Kavallaris, N. Chalvatzas, A. Hornemann, C. Banz, K. Diedrich, A. Agic

University of Schleswig-Holstein, Campus Luebeck, Germany

Introduction: Endometriosis with bowel involvement is a most invasive form and can cause infertility, chronic pelvic pain and bowel symptoms. Effective surgical treatment of endometriosis requires complete excision of endometriosis and in same case may require segmental rectosigmoid resection. Patients and Methods: Between 12/1997 and 10/ 200355 patients with rectovaginal endometriosis underwent a combined laparoscopic vaginal technique. 30 patients were found at a follow up and underwent a telephone interview. The questionnaire covered questions about symptoms related to a recurences of intestinal endometriosis, dyspareunia, dysmenorrhea and pregnancy.

Results: 27 of $30(90 \%)$ women have no clinical symptoms of recurrence of endometriosis. Two patients $(6.6 \%)$ reported recurrence of bowel endometriosis. Dysmenorrhoea disappeared in $28(93.3 \%)$, dyspareunia in $26(86.7 \%)$ and pelvic 
pain in $27(90 \%)$ patients. 17 patients $(31 \%)$ tried to become pregnant and 11 of these patients $(65 \%)$ became pregnant: 9 patients delivered healthy newborns, 18 pregnancies were reported and 19 healthy children were born.

Discussion; Despite the small number of follow up patients, our 94 months follow up data demonstrated that endometriosis with bowel involvement and radical resection was associated with significant reductions in painful and dysfunctional symptoms, a low recurrence rate $(6.6 \%)$ and high pregnancy rate $(36.6 \%)$.

Key-words: deep infiltrating endometriosis, laparoscopy, follow up.

\section{P4_2}

\section{Acute necrosis following endometrioma vaporization by plasma energy is not harmful for underlying ovarian tissue \\ H. Roman, O. Tarta, I. Pura \\ Rouen University Hospital, France}

Background: To evaluate the depth of necrosis induced by vaporisation of the inner layer of ovarian endometriomas using plasma energy.

Methods: A prospective pilot study included women undergoing surgical management of ovarian endometriomas of diameter exceeding $30 \mathrm{~mm}$. The original point of endometrioma invagination was identified for each patient, and the cyst was opened without incision of the ovarian parenchyma. Complete vaporisation of the internal surface of the endometrioma was performed, with or without the inversion of the cyst using plasma energy at setting 40, in coagulation mode, limiting the time of application to 1 to 2 seconds on each site. A cystectomy was then performed and the cyst specimen was referred for histological examination in order to obtain an estimation of the depth of necrosis induced by the vaporisation and evaluate the effectiveness of endometrial tissue ablation.

Results: Ten ovarian endometriomas were vaporised, and subsequently excised in 8 cases. The mean area of necrosis at its largest section was $6.4 \mathrm{~mm}^{2}$ (SD $\left.2.6 \mathrm{~mm}^{2}\right)$, while the mean area of the cyst wall was $29.4 \mathrm{~mm}^{2}$ (SD $9.4 \mathrm{~mm}^{2}$ ), resulting in a necrosis : cyst wall ratio of $0.22 \pm 0.06$. The mean depth of necrosis induced by the vaporisation was $163 \mu \mathrm{m}$ (range 48$1.029 \mu \mathrm{m})$, which was inferior to the fibrous cyst wall width (mean value $632 \mu \mathrm{m}$, range 230-1.105 $\mu \mathrm{m}$ ). Residual endometrial epithelium was identified in 4 cases and concerned less than $10 \%$ of the surface of the cyst inner layer. Necrosis involved underlying ovarian parenchyma in 7 cases, and represented less than $10 \%$ of the ovarian parenchyma inadvertently excised during cystectomy.
Conclusion: Vaporisation of ovarian endometrioma using plasma energy allows the ablation of endometrial tissue with minimal damage to the ovarian parenchyma. Further studies are required to compare this technique to cystectomy, especially in women with risk factors of postoperative ovarian reserve impairment, such as recurrent and bilateral endometriomas. Key-words: ovarian endometrioma, vaporisation, plasma energy

\section{P4 3}

Postoperative medication after laparoscopic ovarian cystectomy reduced the recurrence of ovarian endometrioma

T. Kusakari*, Y. Tsuruta**, A. Kozawa*, H. Suzuki*, N. Tastumi*

*Palmore Hospital, Japan **Department of Medicine, University of Alabama at Birmingham, USA

Introduction: One-third to half of endometriosis patients who initially receives a surgery experiences the secondary surgery because endometriosis recurs. It is an urgent need to identify risk factors for recurrence of endometriosis particularly after laparoscopic ovarian cystectomy.

Materials \& Methods: 30 endometrioma patients, undertaken laparoscopic ovarian cystectomy and subsequent observation for over 6 months with or without postoperative medication, were retrospectively analyzed for recurrence of endometrioma. Recurrence was defined as a newly detected endometrioma which sized more than $2 \mathrm{~cm}$ in MRI diagnosis. We analyzed the correlation between recurrence and 6 factors: age, revised American Society for Reproductive Medicine (rASRM) score, size and laterality of the original endometrioma, postoperative medication, and serum CA125.

Results: The follow-up period after laparoscopic ovarian cystectomy was $17.6 \pm 8.7$ months. Recurrence has appeared in 7 patients out of $30(23.3 \%)$ within $11.7 \pm 2.1$ months observation. All 7 patients who recurred were among 18 cases of no medication group. In contrast, no recurrence has reported in the medication group ( $7 / 18$ vs. $0 / 12, p<0.05)$. The recurrence group has demonstrated higher r-ASRM score and larger endometrioma in the initial diagnosis compared with non-recurrence group $(58.4 \pm 24.9$ vs. $28.3 \pm 6.5, \mathrm{p}<0.05$ and $6.6 \pm 1.6 \mathrm{~cm}$ vs. $4.0 \pm 1.2 \mathrm{~cm}, \mathrm{p}<0.01$, respectively). The other factors were not significantly correlated with recurrence. Discussion: Postoperative medication could contribute to reduce recurrence of endometrioma after laparoscopic ovarian cystectomy, especially when recurrence is prospected with high r-ASRM score and large endometrioma in the initial diagnosis.

Key-words: endometriosis, recurrence, risk factors. 


\section{P4 4}

Pregnancy rate following laparoscopic surgery in infertile women with endometriosis

D. Kwon

St. Vincent's Hospital, The Catholic University of Korea, South Korea

Objective: To investigate the pregnancy rate following laparoscopic surgery in infertile women with endometriosis. Materials and methods: Respective analysis was performed in 54 infertile patients with endometriosis who had operative laparoscopy at St. Vincent's Hospital from January 2004 to February 2010. We investigated the pregnancy rate during 12 months following laparoscopic surgery.

Results: The overall spontaneous intrauterine pregnancy rate during 12 months was $44.4 \%(24 / 54)$. Most of pregnancy occurred during the 3 months after surgery(19/ 24). There was no correlation between spontaneous pregnancy rate and the revised American Fertility Society classification or laparoscopic findings. Conclusion: Laparoscopic surgery may be an effective treatment for infertile patients with endometriosis and most of spontaneous pregnancy occurred during the 3 months after surgery.

Key-words: endometriosis, infertility, laparoscopic surgery.

\section{P4_5}

Incidence of endometrial cysts in laparoscopic surgery of ovaries and surgical treatment

V. Soldo, N. Cutura, M. Vasiljevic, A. Curkovic

Department of Laparoscopic Surgery, G/O Clinic Narodni

Front, Belgrade, Serbia

Introduction: Laparoscopic surgery (LS) has been increasingly replacing classical surgical techniques, depending of course, on pathology, urgency, available technical equipment, and the staff expertise. The following reasons make laparoscopic surgery superior over the classic one: lesser trauma of the anterior abdominal wall, less intensive postsurgical pain, reduced duration of hospitalization and faster recovery, which all together reduce the cost of treatment. However, the duration of surgical procedure itself may be somewhat longer.

Aim: We studied the incidence of particular types of surgical procedures of the ovaries, with a special attention paid at endometrial cysts, and their surgical treatment.

Material and Methods:This was a retrospective study, encompassing a two-year period, from 2007-2008, with 1130 LS performed at the Clinic for Gynaecology and Obstetrics "Narodni front", Belgrade. Among that number, $370(32.74 \%)$ were surgeries of the ovaries. Surgical team included 3 members: an anestesiologist, an anestetician, and the medical-surgical nurse. The following equipement was applied: Olympus (EvisExero II CV-180; UH 1-3; VES40), Storz (Endoscope; Morselator), DVD-Pioneer (HDDDVD recorder; DVR-555H), ETHICON-Ultrasound.

Results: Surgery of the ovaries represents $1 / 3$ of all laparoscopic surgeries, and $370(32.74 \%)$ of 1130 LS that we performed. The structure of surgical procedures was as follows: 202 (54.6\%) were cystectomies, 134 (36.21\%) were cystotomies, and represented 366 or $90.82 \%$ of total LS of ovaries. The rest of LS were: biopsies $20(5.40 \%)$, ovarian drill $10(2.70 \%)$, and ovariectomy and adnexectomy, 2 each, or $0.54 \%$. Average age of the patients who underwent surgery was 31 . The youngest patient was 13 , and the oldest was 45 . Majority of patients $(90 \%)$ were treated for sterility. Hystopathological tests after surgery exhibited following pathological findings: 220 (59.45\%) were endometriomas, 99 (26.75\%) were serous cysts, 22 (5.94\%) were PCO, 16 (4.32\%) were dermoid cysts, and 13 (3.51\%) were hemorrhagic cysts. None of the hystopathological findings was malignant.

Discussion: Benign tumors of the ovaries and PCO syndrome should be treated laparoscopically. Classical laparotomy should be applied for malignant tumors and massive benign tumors, particularly if they are associated with adhesions. We removed minor or major adhesions in $30.18 \%$ of our patients who underwent surgery. In endometriomas of the ovaries, cystectomy, combined with triptorelin (diphereline) results in less recidives and better chance for pregnancy in the first year following surgery. Conclusion: LS should be considered a golden standard for surgery of benign ovarian tumors and PCO syndroma. Malignant ovarian tumors, and massive malignant tumors, as well as benign tumors with adhesions, require classical laparotomy. Majority of tumors ( 220 or $59.45 \%$ ) treated in our group were endometriomas.

Key-words: endometriomas, laparoscopic surgery, hystopatological test.

\section{P4_6}

Results of complications, quality of life and fertility following 106 consecutive cases of women who underwent anterior rectal resection for severe endometriosis

J. Lo, J. English, D. Morgan, J. Dilley

Worthing Hospital, Worthing, UK

Objective: To assess the complications, quality of life and outcome of fertility in women who had anterior rectal resection for severe endometriosis.

Method: Review of medical notes for complications in 106 women who had anterior rectal resection over a 10 year 
duration. Follow up questionnaires assessing quality of life, pain scores and fertility outcomes for a duration of up to 4 years.

Results: Over $75 \%$ had anterior rectal resection were performed entirely laparoscopically. Mean inpatient stay was 7 days. Overall there was no complication in $66 \%$ of women. Fertility outcome: In the last 61 women included in the study, 41 women retained their uterus and of these 36 returned the questionnaire. Twenty women tried to conceive. Nine women reported a positive pregnancy test. Six women had successful live births: 5 women delivered singleton babies, one woman had a set of twins, and one woman is 24 weeks pregnant at the time of this report, 2 had miscarried. Quality of life: Overall 74 women returned the questionnaire with regards to quality of life: 63 [85\%] felt their pain had completely gone or was greatly improved.

Discussion: Anterior rectal resection for severe endometriosis appears to be a successful treatment for this entity. Key-words: severe endometriosis, anterior rectal resection, complication.

\section{P4_7}

\section{Laparoscopic removal of endometriomas - a one year experience \\ M. Simões, F. Ribeiro, C. Marques, S. Barreto, G. Pinto \\ Maternidade Dr. Alfredo da Costa, Lisboa, Portugal}

Summary: Treatment for mild and moderate endometriosis is controversial, whereas ovarian endometriomas of diameter $>3 \mathrm{~cm}$ must be treated surgically, according to some authors. The aim of this retrospective study was to assess among other factors the operative time and safety of laparoscopic removal of endometriomas.

Introduction: A minimally invasive and inexpensive surgical approach should be always preferred, specially when our primary goal is preserving women's fertility. Laparoscopic surgery for the management of ovarian endometriomas has therefore been considered as the gold standard treatment.

Material and Methods: Review including 2 study groups originated from 93 patients who underwent a laparoscopic ovarian cystectomy during 2009: group A which included 26 endometriomas and group B including 67 cysts. We compared several factors, using SPSS version 17.0 statistical software.

Results: Group A had a mean age of 32.04 and group B of 36.43 years old. CA 125 level serum was 62.63 and 20.91 in group $\mathrm{A}$ and $\mathrm{B}$, respectively. Operative time in group A was superior to group B (86.62 versus 63.05$)$. The proportion of nulliparous and of patients with history of infertility was superior in group A. All these differences had statistical significance.

Discussion: Surgical skill in laparoscopy is essential in the treatment of endometriomas, in order to reduce operative time and risk of complications. Surgical intervention is controversial in asymptomatic women with endometriomas. Key-words: endometriomas, laparoscopic cystectomy, operative time.

\section{P4_8}

Comparison between resection, bipolar coagulation and Plasmajet: a preliminary animal study

N. Bourdel, H. Roman, A.S. Azuar, E. Wassim, P. Dechelotte, R. Botchorishvili, G. Mage, M. Canis CHU Clermont-Ferrand, France

Objective: To compare the most used types of surgical techniques, for peritoneal lesions management, to Plasmajet (PJ), in term of wound and post-operative adhesion.

Material and Method: Female pigs were anesthetized and mechanically ventilated. A $\mathrm{CO} 2$ pneumoperitoneum was created. Three trocars were introduced. Eleven areas of $2 \mathrm{~cm} 2$ were treated in each lateral side of the peritoneal wall. Two areas of control, 2 of surgical resection, 2 of bipolar coagulation, 2 of Plasmajet 10 low (PJ10L, adjustment of the Plasmajet) used close to the peritoneum, 2 of PJ10L used at $3-5 \mathrm{~mm}$ from the peritoneum, 2 of PJ10L used at $10 \mathrm{~mm}$ from the peritoneum, 2 of PJ used at 10 High $(\mathrm{PJ} 10 \mathrm{H})$ close to the peritoneum, 2 of $\mathrm{PJ} 10 \mathrm{H}$ used at $3-5 \mathrm{~mm}$ from the peritoneum, 2 of $\mathrm{PJ} 10 \mathrm{H}$ used at $10 \mathrm{~mm}$ from the peritoneum, 2 of PJ used at 40 Low (PJ40L) used at $3-5 \mathrm{~mm}$ from the peritoneum, 2 of PJ40L used at $10 \mathrm{~mm}$ from the peritoneum. For each 2 areas, one was removed for histological analysis. All animals were reoperated 14 days later to evaluate macroscopic healing and adhesion score.

Results: Initial histological analysis shows that in every treated area the peritoneum was completely destroyed or coagulated. After resection, the healing was macroscopically perfect and there was no adhesion, as in the control area. After bipolar coagulation in half of cases there was adhesion. There was no adhesion after treatment by Plasmajet 10 low used at $10 \mathrm{~mm}$ from the peritoneum.

Discussion-Conclusion: This preliminary study shows the difference in term of healing and post-operative adhesion between the different surgical types of destruction of the peritoneum. The use of Plasmajet could be a way of research to find the less aggressive method to destroy the peritoneal endometriosis or carcinomatosis.

Key-words: endometriosis, surgery, adhesion 


\section{P4 9}

Long-term results of laparoscopic resection of deep pelvic endometriosis with rectosigmoid involvement

J. Dowaji, F. Jaenicke

University Medical Center Hamburg-Eppendorf, Germany

Introduction: The purpose of this study was to assess the long-term surgical and functional results of complete laparoscopic resection of deep pelvic endometriosis with bowel involvement.

Materials and Methods: 120 women with severe endometriosis and bowel involvement from 2000 to 2005 were identified from a prospective database and were retrospectively analyzed. Laparoscopic excision of all visible disease was planned. Data analysis included age, body mass index, previous history of endometriosis, pregnancies, operative procedure, intra- and postoperative complications, pain relief and disease recurrence after a follow-up of at least 5 years. Results: The median age of patients was 35 years. Some of patients with history of previous operation (50\%). Preoperative symptoms were present as dysmenorrhea (90\%), dyspareunia (73\%). rectal pain (52\%), constipation (43\%), rectal bleeding (28\%). Management of the bowel disease included superficial excision of serosal endometriosis implants $(n=21)$, disc excision $(n=19)$, rectal resection with low rectal $(n=73)$ or coloanal $(n=7)$ anastomosis. Twenty five cases were converted to laparotomy. Postoperative complications occurred in $15 \%$. Twenty patients developed a transient neurogenic bladder. A fertilitypreserving procedure was possible in 101 patients. Only 19 patients with a uterus required laparoscopic hysterectomy or bilateral salpingo-oophorectomy. All patients reported a clinically significant improvement of their symptoms with no recurrence of bowel endometriosis.

Discussion: Complete radical laparoscopic excision of endometriosis is feasible and provides excellent long-term surgical and functional results.

Key-words: endometriosis, laparoscopy, anastomosis.

\section{P4_10}

The use of $\mathrm{JJ}$ stent in the laparoscopic management of ureteral endometriosis

S. Anglès, M.A. Martínez-Zamora, I. González, F. Carmona, A. Franco, J. Balasch

Institut Clínic de Ginecologia, Obstetrícia i Neonatologia. Institut Clínic de Nefrologia i Urologia. Hospital Clínic of Barcelona. University of Barcelona, Spain

Summary: The use of JJ stent in the laparoscopic treatment of UE can be useful to treat and prevent ureteral injuries.
Introduction: Laparoscopic surgery is the treatment of choice of ureteral endometriosis (UE) although can be associated to technical difficulties. We reported our experience in the laparoscopic management of UE using JJ stents.

Methods: Retrospective analysis of our series of 112 patients with deep infiltrating endometriosis.

Results: 29 patients had histological proved UE (16 left, 8 right and 5 bilateral). All patients had severe implants of endometriosis in other sites of the pelvis and in all patients a JJ stent was placed in one or both ureters before or during surgery. In all patients a complete surgical excision of endometriosis was performed. Histological results showed severe infiltrating UE in 18 patients (62\%). Ten patients $(34.5 \%)$ presented with severe UE which caused moderate to severe hydroureteronephrosis (7 left, 2 right and 1 bilateral). In those patients who were presurgically diagnosed with ureteral stenosis the JJ ureteral stents were placed before surgery to preserve kidney function. Treatment consisted of ureteroneocystostomy in 7 patients, ureteral resection and end-to-end anastomosis in 6 patients and ureterolysis in 16 patients. In all patients a laparoscopy was initially performed. In nine patients $(31 \%)$ conversions were performed due to severe adhesions and/or intestinal resection, but not due to ureteral complications. No major complications occurred during or after surgery.

Discussion: The use of JJ stent in the laparoscopic treatment of UE can be useful to prevent ureteral injuries, avoid conversions to laparotomy, maintain ureteral integrity after ureteral resections or ureteral surgical injuries, and can treat ureteral stenosis causing hydronephrosis before surgery.

Key-words: ureteral endometriosis, JJ stent, deep infiltrating endometriosis.

\section{P4_11}

Denovo vaginal vault endometrioma excised with the McCartney Tube: a simple novel technique R. Laiyemo, M. Nemcova, S. Disu, S.O. Porter Airedale General Hospital, Steeton West Yorkshire \& St Mary's Hospital, Paddington, London, UK

The presence of de-novo endometriosis in the vaginal vault is rare in post hysterectomised women with no previous clinical evidence of endometriosis. The association with the use of prolonged hormone replacement therapy is also rare but is aetiologically feasible. We present a case of the novel use of the McCartney tube to excise a vault endometrioma in a 39 year old woman presenting with de-novo vault disease. This we believe represents a simple effective way of managing this rare condition in a way that minimizes patient morbidity. 
Introduction. The McCartney tube has long been established as a means of exteriorizing lymph nodes during laparoscopic pelvic lymphadenectomy (McCartney AJ et al 1995) and for the maintenance of pneumo-peritoneum during laparoscopic hysterectomy (McCartney et al, 2004). A PubMed search yielded no results for "chronic pelvic pain, vaginal vault excision, endometriosis and McCartney tube", making this, to the best of our knowledge, the first case report of vaginal vault excision of an endomtrioma with the McCartney tube.

Case Report: A 39 -year old Para 3 Caucasian woman presented with a one year history of pelvic pain dyspareunia and occasional per vaginal spotting. She had had a total abdominal hysterectomy for pelvic pain 12 years previously, followed by a bilateral salpingo-oophorectomy 2 years later for chronic pelvic pain. Histolopathological examination of the uterus, cervix and ovaries did not reveal any evidence of endometriosis. She was then commenced on oestrogen only hormone replacement therapy which she remained on until presentation. EUA confirmed a $2 \times 3 \mathrm{~cm}$ firm structure (scar tissue) at the vault and a vault biopsy confirmed endometriosis on histology. The patient was then referred for the minimally invasive surgery. Findings at therapeutic laparoscopy included extensive pelvic adhesions overlying the vault and left pelvic side walls. Adhesiolysis was performed. A $35 \mathrm{~mm}$ McCartney tube, was inserted into the vagina and the vault containing the endometrioma was excised laparoscopically. Vault closure was laparoscopic. The woman made an uneventful recovery and was well at three month review.

Discussion: This is the first case report of the use of a McCartney tube for excision of de-novo endometriosis in the vaginal vault of a post hysterectomised woman. Traditionally vault excision has been done by laparotomy and more recently by laparoscopy however most laparoscopic methods use sponge holding forceps to raise the vaginal apex to allow easier visualization of the apical area whilst using a glove or gauze in the vagina to maintain the pneumoperitoneum after excision of the vaginal vault. This method is cumbersome and time consuming. Using the McCartney tube as described above negates the need to use cuffs or to pack the vagina with gloves and gauze in order to maintain the pneumoperitoneum during vaginal vault excision. It also avoids the need to use sponge forceps to raise the vaginal apex before excision. In addition, prior to excision the lesion can be accurately identified by placing the laparoscope into the distal end of the McCartney tube while the latter is in the vagina. Furthermore, the tube provides a conduit for safe laparoscopic removal of surgical specimens removed at laparoscopy. The McCartney tube therefore provides an effective and simple means of addressing vaginal vault pathology (endometriosis in this case) which is efficient and involves minimal patient morbidity.
Key-words: McCartney tube, endometriosis, chronic pelvic pain.

\section{P4 12}

Laparoscopic management of ovarian endometriomas F. Sendag, B. Zeybek, V. Turan, K. Oztekin, O. Bilgin Ege University School of Medicine, Turkey

Summary: Today it is well known that medical therapy alone is not effective in reducing endometriotic cysts and laparoscopic surgery is the gold standard treatment for ovarian endometriomas. The aim of the present study was to analyse 67 consecutive patients with ovarian endometriomas who were managed via laparoscopy and review the literature regarding the management of endometriotic cysts. Introduction: Endometriosis is a disease in which endometrial glands and stroma are found outside the endometrial cavity. The incidence of endometriosis ranges from $2 \%$ $22 \%$ in asymptomatic women, but $40 \%-60 \%$ in patients with dysmenorrhea, and $20 \%-30 \%$ in those with infertility (1-3). Endometriomas are the third most common manifestation of endometriosis after Douglas peritoneum and uterosacral ligament endometriosis, and represent $35 \%$ of benign ovarian cysts requiring surgery (4). The aim of the present study was to analyse 67 consecutive patients with ovarian endometriomas who were managed via laparoscopy and review the literature regarding the management of endometriotic cysts.

Materials and Methods: One hundred forty-eight patients who underwent surgery in the Obstetrics and Gynaecology Department of Ege University School of Medicine in Izmir, Turkey due to adnexal massed and confirmed histologic diagnoses of endometriomas between January 2005 and November 2008 were analysed retrospectively. Before surgery, all patients were evaluated with pelvic examinations, transvaginal ultrasonography, and CA-125 levels. The exclusion criteria were as follows: pregnancy, history of cancer, use of estrogen suppressive drugs (oral contraseptives, GnRH analogues, progestins, or danazol in the preceding 6 months before surgery), and lack of data. Sixtyseven patients who met all the criteria were included in the analysis. Peripheral blood samples were collected from all the patients in the early follicular phase. The CA-125 level was measured in duplicate using an OM-MA Immulite 2000 kit (DPC; Diagnostic Products Corporation, Los Angeles, CA, USA) by the chemiluminescence method. Operative laparoscopy was performed during the late proliferative phase of the cycle through insertion of a 10-mm subumbilical trocar and three 5-mm trocars, two of which were in the lower abdomen, and one of which was in the line with the umbilicus and and the left lower trocar. All 
laparoscopic procedures were performed by the same laparoscopist. In all patients, the diagnosis was confirmed both laparoscopically and histologically. Endometriosis was staged according to the revised American Society for Reproductive Medicine (rAFS) classification (5). After identification of the cleavage plane, the wall of the cyst was stripped from the healthy surrounding normal ovarian tissue with the use of two atraumatic grasping forceps. Hemostasis was achieved with application of a $50-\mathrm{W}$ current using bipolar forceps to the cyst bed.

Results: The mean age of the patients was $29.6 \pm 5.3$ years $(n=67)$. Thirty-one percent of the patients $(21 / 67)$ did not have any complaints and the initial diagnosis was made during a routine annual gynecologic ultrasound examination. Fifty-five percent (37/67) of the patients had dysmenorrhea as a presenting symptom, whereas $11 \%$ of the patients $(7 / 67)$ had a complaint of infertility, and $3 \%$ of the patients $(2 / 67)$ had abnormal bleeding. The mean size of the endometriomas was $52 \pm 20 \mathrm{~mm}$ (range, $30-150 \mathrm{~mm}$ ). There were bilateral endometriomas in $30 \%$ of the patients $(20 / 67)$, whereas $54 \%$ of the patients $(36 / 67)$ had an endometrioma on the left side and $16 \%$ of the patients $(11 / 67)$ had an endometrioma on the right side. The other implantation sites in the abdomen are shown in Table 1 . The mean CA-125 level was $117.4 \pm 266 \mathrm{IU} / \mathrm{ml}$ (range, 15 $1966 \mathrm{IU} / \mathrm{ml})$. The patient with a CA-125 level of $1966 \mathrm{IU} /$ $\mathrm{ml}$ was admitted with symptoms of an acute abdomen, and during laparoscopy rupture of an endometrioma was noted. According to the rAFS classification, $22 \%$ of the patients $(15 / 67)$ were stage $1,39 \%(26 / 67)$ were stage $2,25 \%(17 /$ $67)$ were stage 3 , and $14 \%(9 / 67)$ were stage 4 . Laparoscopic cystectomies were performed on all patients. None of the patients required conversion of the laparoscopic procedure to a laparotomy. No intra- or post-operative complications occurred. Table 1. Other endometriotic implantation sites in the abdomen $(n=67)$ None (n) 32 (48\%) Peritoneal endometriosis (n) 20 (30\%) Bladder endometriosis (n) 1 (1\%) Sigmoid colon endometriosis (n) 1 (1\%) Peritoneal+bladder endometriosis (n) 7 (10\%) Bladder+bowel (n) 0 Peritoneal+bowel (n) 1 (1\%) Bladder+peritoneal+bowel (n) 5 (7\%).

Discussion: Both laparoscopy and laparotomy have the same results in terms of pregnancy and recurrence rates, but laparoscopy is considered to be the best approach because of less blood loss during the operation, a reduced need for analgesia post-operatively, a shorter length of hospitalization, and a shorter length of recovery. In a meta-analysis, comparing the outcome of laparoscopic surgery and laparotomy, it was shown that both techniques exposed patients equally to complications (6). However, a laparotomy must be kept in mind as an alternative option for cases of severe endometrosis associated with dense extensive adhesions and deeply infiltrating endometriosis. There are four main techniques described for the conservative laparoscopic treatment of endometriomas: 1) laparoscopic aspiration; 2) cystectomy; 3 ) drainge and destruction of the inner lining with an energy modality; and 4) three-stage management. The main problem with laparoscopic cyst drainage is an $80-100 \%$ risk of recurrence $(7,8)$. The administration of GnRH agonists after drainage reduces the size of the cyst up to $50 \%$, (9) but GnRH agonists have no effect on the recurrence rate (10). Therefore, it is accepted that laparoscopic cyst drainage should not be used to treat endometriomas. Although there have been some controversies on inducing damage to the ovarian reserve $(11,12)$, laparoscopic cystectomy remains a first-line treatment for endometriotic cysts. With this technique, the wall of the cyst is stripped from the healthy surrounding normal ovarian tissue with the use of two atraumatic grasping forceps after identification of the cleavage plane. The reported recurrence rates after cystectomy vary between $4.8 \%$ and $17.3 \%(13,14)$. In a most detailed study by Busacca et al. (25) the risk factors for the recurrence of endometriomas following laparoscopic cystectomy were examined; three risk factors were suggested, including a previous history of endometriosis, stage 4 rAFS classification, and rAFS scores. The controversy about the ovarian reserve damage of the cystectomy technique has led some surgeons to use the technique of laparoscopic ablation. In a recent Cochrane meta-analysis (15) comparing the two techniques for endometriomas (cystectomy vs. ablative method), laparoscopic excision of the cyst wall of the endometrioma was associated with a reduced recurrence rate of symptoms of dysmenorrhea (OR, 0.15; CI, 0.060.38 ), dyspareunia (OR, 0.08; CI, 0.01-0.51), nonmenstrual pelvic pain (OR, 0.10; CI, 0.02-0.56), a reduced rate of recurrence of the endometrioma $(\mathrm{OR}, 0.41$; CI, 0.180.93 ), and a reduced requirement for further surgery (OR, 0.21; CI, 0.05-0.79). Laparoscopic cystectomy was also associated with a subsequent increased spontaneous pregnancy rate in women with subfertility (OR, 5.21; CI, 2.0413.29), and an increased ovarian follicular response to gonadotrophin stimulation (WMD, 0.6; CI, 0.04-1.16). With respect to the chance of pregnancy after controlled ovarian stimulation, the authors concluded that there is insufficient evidence to favour excisional surgery over ablative surgery with respect to the chance of pregnancy after controlled ovarian stimulation and intra-uterine insemination (OR, 1.40; CI, 0.47-4.15). The three-stage technique for the conservative treatment of ovarian endometriomas was first described by Donnez et al. (9). This technique consists of performing simple drainage during the first laparoscopy, followed by GnRH agonist administration for 12 weeks, and vaporization of the inner lining using a $\mathrm{CO} 2$ laser during a second laparoscopy; the post-operative cumulative pregnancy rate is $51 \%$ and the recurrence rate 
is $8 \%$ during a follow-up of $2-11$ years. Due to data in the literature, it is concluded that ovarian cystectomy is associated with diminished ovarian reserve in women of reproductive age, and follicular recruitment of the treated ovary is improved after laser vaporization (4). In conclusion Endometriomas are frequent ovarian cystic structures encountered by gynaecologists. In conservative management, laparoscopic cystectomy is an effective and safe procedure when compared with laparotomy. However, further studies with large, diverse patient populations are needed to compare laparoscopic techniques.

Key-words: endometrioma, laparoscopy, cystectomy.

\section{P4 13}

\section{Endometriosis surgery or IVF:which is effective}

G. Guney, T. Var, E. Tonguc, E. Isci, S. Batioglu

Zekai Tahir Burak Women Health Education and Resarch Hospital, Turkey

Introduction: The aim of this study is to evaluate the spontaneus pregnancy rates of the patients with endometriosis after laparoscopic surgery and to asses the IVF outcome of the patients who could not conceive spontaneously.

Materials and Methods: In this study, 141 primary infertile patients were performed laparoscopic endometriosis surgery. After surgery patients were followed for a period of 1 to 8 years and 95 patients who could not conceive postoperatively were performed IVF-ET.

Results: The number of patients with Stage 1 and Stage 2 endometriosis were $59(41.8 \%)$. The number of patients with Stage 3 and Stage 4 endometriosis were 65 (68.4\%). While the total pregnancy rate was $56,7 \%, 46$ of 141 (32.6\%) patients who had laparoscopic surgery conceived spontaneously. When analyzed in terms of spontaneous pregnancy, the number of patients who conceived spontaneously at Stage 1 and Stage 2 were 29 (49.2 \%) whereas the number of patients who conceived spontaneously at Stage 3 and Stage 4 were 17 (20,7\%9). For all patients the pregnancy rate was $45,7 \%$ at the end of 12 th month, 54,6 $\%$ at the end of 24th month and 57,4 \% after the 34th month. After 34th month the pregnancy rates reached plateau and remained constant afterwards. Kaplan-Meier Curves were used to show the Cumulative Pregnancy Rates for All Patients and the Cumulative Pregnancy Rates According to Age and stage of the disease. In Stage 1 and Stage 2 patients, the pregnancy rates were $44.8 \%$ at the end of the 6th month, $76.5 \%$ at the end of 24 th month. At the end of 34th month, the pregnancy rate was $79.1 \%$ and afterwards it remained constant. As for Stage 3 and Stage 4 patients, the pregnancy rate was $15 \%$ at the end of 6 th month, $39.3 \%$ at the end of 24th month. At the end of 29th month, the pregnancy rate was 42.3 and afterwards it remained constant. Cumulative pregnancy rates at Stage 1 and Stage $2(79.1 \%)$ were found to be significantly higher than pregnancy rates at Stage 3 and Stage 4 (42.3\%). The pregnancy rate at Stage 3 and 4 were found to be significantly lower than Stage 1 and Stage $2(\mathrm{p}<0,001)$. The total pregnancy rate at Stage 1 and 2 was $76.3 \%$ whereas the pregnancy rate was $42.7 \%$ at Stage 3 and $4(\mathrm{p}<$ $0.001)$. the pregnancy rates at Stage 1 and 2 are more than Stage 3 and 4 . The conception rate $(27,5 \%)$ in all patients within the first six months after laparoscopy was found to be higher than the other months $(\mathrm{p}<0,001)$. Moreover, the fecundity rate in women older than 35 years old was statistically and significantly lower than women under the age of $35(\mathrm{p}=0,003)$.

Conclusion: Surgical treatment was found to be effective for the patients with endometriosis associated infertility. IVF-ET could be suggested for patients who could not conceive spontaneously within first 12 months after the surgery. Key-words: endometriosis, laporoscopic surgery, IVF.

\section{P4_14}

\section{Does surgical treatment prior to GnRH agonist therapy improve outcome in Ovarian Endometriosis (OE) - associated infertility?}

L. Ples, M. Burtea, C. Ignat

Bucur Maternity, St Ioan Clinical Emergency Hospital, Bucharest, Romania

Background: Although the therapy of OE-associated infertility is based either on spontaneous conception after conservative surgery or pseudomenopause induction by GnRH-agonist, these 2 combined improve the fertile outcome of OE. The aim of this study is to determine the efficiency of combined therapy in OE-associated infertility. Material and Methods: A retrospective study was carried out in patients with $\mathrm{OE}$ who underwent surgical and medical therapy in last 2 years.

Results: Out of 52 patients $30(57.7 \%)$ have been treated postopearative with GnRH-agonist (Dipherelin0.75 mg). Mean age was $31.2 \pm 6.9$ years. Symptoms at presentation: dysmenorrhea and dyspareunia-100\%cases, infertility$42.3 \%$ cases, menorrhagia- $11.5 \%$ cases with a $92.4 \%$ correlation of pre-postoperative diagnosis. We performed both laparoscopic and open surgery: 24 classic cystectomies, 10 (29.4\%) laparoscopic cystectomies, 4(3.5\%) ovariectomies, $10(19.2 \%)$ adnexectomies and $4(11.6 \%)$ total hysterectomies with bilateral adnexectomy. $88.2 \%$ of patients undergoing cystectomy followed 6 months GnRH agonist therapy with complete symptoms remission and significant 
difference regarding spontaneous conceptions unlike patients undergoing surgery alone.

Conclusions: The incidence of spontaneous conception after combined therapy was $40 \%$. Although a long term therapy, GnRH agonist subsequently to conservative surgery offers an improvement of the fertile outcome of $\mathrm{OE}$ unlike cases undergoing surgery alone with an incidence of $15 \%$ spontaneous conception.

Key-words: ovarian endometriosis associated infertility, combined therapy, conservative surgery.

\section{P4_15}

\section{Prevalence of hypoactive sexual desire disorder in patients with rectovaginal endometriosis}

J.L. Coloma, M.A. Martínez-Zamora, F. Carmona, C. Castelo-Branco, J. Balasch

Institut Clínic de Ginecologia, Obstetrícia i Neonatologia. Hospital Clinic of Barcelona. University of Barcelona, Spain

Summary: The laparoscopic excision of rectovaginal endometriosis (RVE) improves and normalises the quality of sex life 4-6 months after surgery. Introduction: Women with RVE are at high risk of developing hypoactive sexual desire disorder (HSDD), which may cause sexual and emotional discomfort. We determined the prevalence of HSDD in patients with RVE and assess the effect of its laparoscopic treatment. Methods: Observational prospective study including 36 sexually active women with RVE suffering severe deep dispareunia (DD) and 20 healthy controls. All patients were premenopausal and no patient was treated with analogues of GnRH. Patients underwent laparoscopic full excision of RVE. Patients with RVE, before surgery (Group 1) and 46 months after surgery (Group 2), and controls (Group 3) answered The Brief Profile of Female Sexual Function (BPFSF. Scores $<20$ show a hypoactive sexual desire disorder).

Results: The comparison of patients before surgery and controls showed a statistically significant impairment of sexual life in patients with RVE (Mean B-PFSF = Group 1: $20.9 \pm 8.6$; Group 3: 27.9 $\pm 3.5 ; \mathrm{p}<0.001$ ). At $4-6$ months follow-up patients with RVE had a significant improvement of the quality of sexual life (Mean B-PFSF = Group 1: $20.9 \pm 8.6$; Group 2: $24.8 \pm 7.7 ; \mathrm{p}=0.1$ ). There were no statistical differences between Group 2 and 3, demonstrating a normalization of the quality of sexual life after the complete surgical excision of RVE $(p=0.1)$. A total of 14 patients (39\%) in Group 1, $3(8 \%)$ in Group 2 and $0 \%$ in Group 3 presented total scores lower of equal to 20 indicating HSDD.
Discussion: Patients with RVE have an impairment of quality of sex life compared to controls. The results of this pilot study suggest that laparoscopic excision of RVE improves and normalises the quality of sex life 4-6 months after surgery.

Key-words: deep infiltrating endometriosis, sexual life, quality of sexual life.

\section{P4_16}

\section{Complication rates associated with surgery for severe rectovaginal endometriosis: does the approach and pathway matter? A single centre study A. Kent, T. Rockall Minimal Access Therapy Training Unit, Guildford, UK}

Aims: To assess the risk of complex laparoscopic surgery for severe endometriosis and the effect of the surgical approach using the Guildford protocol.

Materials and Methods: Patients who were scheduled for laparoscopic surgery in order to remove deeply infiltrating endometriotic nodules. A combined surgical approach involving both gynaecology and colorectal teams was applied from the outset via an Integrated Care Pathway with the majority of surgery carried out as a 2 stage procedure with intervening GnRH analogue down regulation. Bowel preparation involved a low residue diet for a week with phosphate enemas on the day of admission prior to surgery. The primary methods of removal were excision by Harmonic ACE +/- laser vaporisation. Temporary JJ ureteric stents were employed as required.

Results: 150 patients had surgery for DIE between June 2004 and June 2005. All had severe, extensive endometriosis involving bowel, bladder or ureters. The decision for bowel surgery was individualised and determined at operation based on severity of disease, symptoms and other factors such as the desire for fertility.

\begin{tabular}{lll}
\hline & $\mathrm{n}=150$ & $(\%)$ \\
Laparoscopy & 142 & $(94.7)$ \\
Laparotomy & 8 & $(5.3)$ \\
Conservative & 110 & $(73.3)$ \\
Clearance (inc hysterectomy) & 40 & $(26.7)$ \\
Colostomy (defunctioning at surgery) & 2 & $(1.3)$ \\
Shave & 65 & $(43.4)$ \\
Disc & 9 & $(6)$ \\
Resection & 64 & $(42.6)$ \\
Bladder/ureter & 12 & $(8)$ \\
Immediate complications & 7 & $(4.6)$ \\
Long term complications (stricture) & 6 & $(4.0)$ \\
\hline
\end{tabular}


94.7\% were completed laparoscopically. 74\% were conservative procedures with conservation of uterus and ovaries. Defunctioning colostomy rate was $1.2 \%$. Where bowel surgery was required clearance was achieved by disc resection in $6 \%$ with the rest being equally split between segmental resection and shaves. Immediate complications included 1 ureteric injury. 1 return to theatre with vaginal vault bleeding, 2 anastomotic breakdowns with defunctioning colostomies, 1 gas leak, 1 para-rectal abscess, 1 pulmonary embolus. Longterm complications were 6 anastomotic strictures one of which required repeat segmental resection. $80 \%$ of these patients had fibrosis or endometriosis identifiable in the donuts from the staple gun (margins of excision). This is significantly higher than the rest of the population who had no problems with stricture where the rate is under $18 \%$.

Conclusions: The combined, two stage approach to the surgical excision of recto-vaginal endometriosis allows the majority of procedures to be completed safely, laparoscopically without recourse to covering colostomies. Bowel surgery should be dictated by the severity of disease and individualised to achieve optimal excision so avoiding unnecessary immediate and longterm complications. An indicator of potential problems due to anastomotic stricture may be the presence of fibrosis or endometriosis in the donuts.

\section{Poster Session 5_Hysterectomy}

\section{P5_1}

\section{LAVH: a 7 year experience in a District General Hospital in UK}

H. Al-Hadithi, A. Swaminathan, J. Hawe

Countess of Chester Hospitals NHS Foundation Trust, UK

Out of 1855 hysterectomies performed in the hospital 108 (5.8\%) were done laparoscopically. $98.1 \%$ of the cases were done with the consultant as the primary operating surgeon. Majority $43.5 \%$ were in the age group 4050 years. $11.1 \%$ of the cases had previous LSCS as a complicating factor. The commonest indication was menorrhagia (32.4\%), the second commonest being menorrhagia and dysmenorrhoea (25.9\%). The histology was normal in $40.7 \%$, with adenomyosis in $32.4 \%$ and fibroids in $31.4 \%$. The majority of our patients had a normal sized uterus $(41.7 \%)$ and $25 \%$ had a subjectively bulky uterus. There was no uterine descent in $56.5 \%$ of the patients with $40.7 \%$ having first degree descent. 99.1\% had routine entry using veress needle. Only 1 patient had open Hassan entry technique used. Bipolar grasper/ monopolar scissors were used in $98.1 \%$ cases with harmonic scalpel used in only $1.9 \%$ patients. Bladder injury was identified in $1.9 \%$ of cases with conversion to laparotomy done in $3.7 \%$ (4 patients) of cases. Of the 4 cases which had conversion to laparotomy 2 had bladder injury, one had bleeding and 1 had failed pneumoperitoneum. 96.3\% received post operative thromboprophylaxis with LMWH. Laparoscopic hysterectomy resulted in a lesser hospital stay and also is less painful as already proved by the eVALuate trial. The majority of cases in our series were laparoscopic assisted vaginal hysterectomy. Majority of patients went home on the third postoperative day with a significant reduction in costs for the hospital. $73.1 \%$ of patients were discharged from gynaecological follow up. It is safe and effective. The uptake of the procedure could be increased by training more doctors in this procedure.

Key-words: LAVH, laparotomy, cost reduction.

\section{P5_2}

\section{Transumbilical Single-Port Laparoscopic-assisted} Vaginal Hysterectomy: comparison with conventional three-ports method after adjustment for uterine weight M.H. Jung, Y.I. Ji, B.Y. Lee

Kyung Hee Medical Center, Haeundaepaik Hospital, Republic of Korea

Introduction: Since laparoscopic hysterectomy became the standard approach for most benign uterine diseases, surgeons have developed laparoscopic techniques for many procedures. As the advantages of minimally invasive procedures became evident, even less invasive procedures were envisioned and developed. Transumbilical single port laparoscopic-assisted vaginal hysterectomy (S-LAVH) is one such minimally invasive procedure. This study aimed to evaluate our initial experience with S-LAVH as compared with the more conventional three-port LAVH in women with benign uterine diseases after adjustment for uterine weight by transvaginal sonography.

Material \& Methods: Between April 2009 and April 2010, S-LAVH was attempted on 147 patients with benign uterine diseases through a transumbilical incision for a 12-mm trocar, and conventional three-port LAVH was performed on 193 patients by the same surgeon. A single-port access system, a wound protractor and a surgical glove, was used. Those patients with estimated uterine weights between $200 \mathrm{~g}$ and $400 \mathrm{~g}$ were included in this study (117 vs. 156 patients). The age, body mass index (BMI), operative time, blood loss, and weight of the specimen were compared. 
Results: Two of 117 patients in the S-LAVH group were converted to 2- or 3-port surgery, compared to none in the conventional group. Data analysis was done for 115 patients in the S-LAVH group and 156 patients in the LAVH group. No major complications, including ureteral or bladder injuries, occurred in any of the patients. No statistically significant differences were found in mean age, mean BMI, mean operative time, mean anesthesia time, mean estimated blood loss, or mean post-operative days to passage of flatus. The mean uterine weights on the pathologic reports were also not significantly reduced in the S-LAVH group $(245.09 \pm$ $52.67 \mathrm{~g}$ in S-LAVH vs. $233.06 \pm 58.24 \mathrm{~g}$ in conventional $\mathrm{LAVH}, \mathrm{P}=0.12$ ).

Conclusion: S-LAVH is a feasible alternative method for removal of the uterus with a better cosmetic outcome. But more surgical experience and improvement in instruments suitable for S-LAVH are needed.

Key-words: hysterectomy, single-port laparoscopy, transumbilical.

\section{P5_3}

Total laparoscopic hysterectomy - a consecutive series for 4 years

K.-B. Lee, S.-A. Hwang, S.-Y. Lim, C.-Y. Park

Department of Obstetrics and Gynaecology, Gachon University Gil Hospital, Korea

Objective: To evaluate the outcome after total laparoscopic hysterectomy for benign disease of the uterus. Method: In this retrospective observational study 337 consecutive patients were laparoscoped between March 2006 and March 2010 to perform total laparosopic hysterectomy using the RUMI manipulator and Koh colpotomiser system. Results: Three hundred and thirty six patients were completed laparoscopically, and $1(0.3 \%)$ required a openlaparotomy to remove the uterus. The indication for surgery were uterine fibroids/adenomyosis $(\mathrm{n}=268,79.8 \%)$; carcinoma in situ $(\mathrm{n}=34,10.1 \%)$; cervical intraepithelial neoplasia $(n=21,6.3 \%)$; endometrial hyperplasia $(n=5$, $1.5 \%)$; endometrial polyp $(\mathrm{n}=5,1.5 \%)$; H-mole $(\mathrm{n}=3$, $0.9 \%$ ). The mean age was 45.7(range 34 69); mean uterine weight $415 \mathrm{~g}$ (range 38 1160); mean blood loss $265 \mathrm{ml}$ (range 55 1000); mean hospital stay 4.2 days (range 2 33). Post-operative complications include vaginal vault dehiscence $(n=5,1.5 \%)$, umbilical hernia $(n=1,0.9 \%)$, peritonitis due to sigmoid colon perforation $(n=1,0.9 \%)$, and ureteral injury $(\mathrm{n}=1,0.9 \%)$.

Conclusion: Total laparoscopic hysterectomy is a safe procedure that removes the uterus with benign disease.

Key-words: hysterectomy, laparscopic.

\section{P5 4}

Laparoscopic approach to hysterectomy for benign disease: our experience

L. Mañalich, A. Asturgó, M. Bradbury, T. Guerra, E. Suárez, J. Xercavins

Hospital Universitari Vall d'Hebron, Spain

Objective: To evaluate clinical and surgical results of laparoscopic hysterectomies for benign gynaecological disease carried out in our University Hospital from January 2005 to December 2009, and to compare the results in time. Materials and methods: A retrospective descriptive study was performed reviewing 225 cases of laparoscopic hysterectomies with non-oncologic indication. We collected clinical, surgical and hystopathological data from the medical history. We evaluated all the information doing a descriptive analysis with SPSS 17.0. We compared the median postoperative hospital stay, the rate of operative complications and the rate of conversion to laparotomy in every year of the study.

Results: The mean age was 46,3. The most common indication for hysterectomy was uterine leyomiomas associated to abnormal bleeding $(46,9 \%)$. Of the 225 hysterectomies, $94,7 \%$ were performed by laparoscopic approach (78,4\% supracervical hysterectomies and $21,6 \%$ total hysterectomies) with a rate of conversion to laparotomy of $10,3 \%$; and $5,3 \%$ by laparoscopical assisted vaginal approach. Surgical complications were registered in 34 patients. The median postoperative hospital stay was 2,9 days. Comparing year by year, there were not statistically significant differences in the rate of operative complications and in the rate of conversion to laparotomy. However, significant differences were found in the median postoperative hospital stay, a higher number of patients with a median postoperative hospital stay $\leq 2$ days was observed in time $(2005-25 \%$ versus $2009-73,9 \%, \mathrm{p}=0,0001$ ).

Discussion: The experience in our centre shows that the laparoscopic approach to hysterectomy for benign disease is feasible; and it offers advantages, regarding low surgical complications and lower median postoperative hospital stay. Comparing the results in time, a decrease in the median postoperative hospital stay was noted.

Key-words: laparoscopic hysterectomy, benign gynaecological disease, median postoperative hospital stay.

\section{P5 5}

The effect of uterine weight to the morbidity in total laparoscopic hysterectomy

S.-h Park

Kangnam Sacred Heart Hospital, Hallym University, Korea 
Introduction: The aim of this study is to evaluate the effect of uterine weight on short-term outcome of total laparoscopic hysterectomy(TLH) for benign gynaecological conditions.

Materials and Methods: A retrospective medical records review of 310 cases of patients with TLH was performed. Patients included in this study underwent TLH as benign uterine disorders at Kangnam Sacred Heart Hostpital, Hallym University from January 2008 through April 2010. These patients were stratified into three groups; Group 1 patients with uterus weighing less than $180 \mathrm{~g}(\mathrm{n}=$ 69), Group2 patients with uterus weighing $180 \mathrm{~g}$ to 350 $\mathrm{g}(\mathrm{n}=169)$, Group 3 patients with uterus weighing more than 350 g. $(\mathrm{n}=72)$. The groups were compared as regard postoperative stay, operative time, estimated blood loss, hemoglobin change, conversion to open surgery, and postoperative complications.

Results: There was no significant difference in age, gravidity, body mass index, previous pelvic surgery and past medical history. The overall complication rates were not significantly different. But operative time prolonged as uterine weight increased.

Discussion: The TLH can be performed successfully in case of enlarged uterus. Therefore the enlarged uterus is not an absolute contraindication for TLH.

Key-words: total laparoscopic hysterectomy, uterine weight, morbidity.

\section{P5_6}

\section{Laparoscopic repair of vaginal evisceration after hysterectomy}

M.J. Fernández Ramírez, J. Gilabert Estellés*, E. Reynal Reillo, R. Aliaga Parreño, J.J. Rodenas Palazón, S. Costa Castellá, F. Coloma Colomer, B. Salas Velastegui, M. Martínez Gomez, J. Gilabert Aguilar

Institution: Hospital Arnau de Vilanova. ${ }^{*}$ Hospital Casa de Salud Valencia, Valencia

Introduction: The aim of this paper is to present the feasibility and effectiveness of laparoscopic repair in three patients with spontaneous vaginal evisceration.

Material and Methods: In the first case, the patient presented with evisceration of a $100 \mathrm{~cm}$ long loop of small bowel three weeks after having undergone a radical laparoscopic hysterectomy. In the second case, the patient -who wore a uterine pessary- presented with evisceration two years after undergoing a vaginal hysterectomy. The third case happened seven days after having undergone a total laparoscopic hysterectomy.

Results: Laparoscopic repair in both cases was possible despite the time elapsed after evisceration (4, 6 and 2 hours respectively). After an anesthesic and deep easing the reintroduction of bowels was performed. The vitality of the bowel loops was observed after being reintroduced in the abdominal cavity. The edges of the vagina were refreshed and the vagina was stitched with loose stitches using polyglactin 0 . In both cases, the postoperatory period was asymptomatic and, in the checkups 3 and 6 months later, it was observed that the vaginal vault had completely cicatrized.

Discussion: Vaginal evisceration may occur after a vaginal or laparoscopic hysterectomy. Laparoscopic repair is feasible and straightforward, and this procedure allows the viability of bowel loops.

Key-words: vaginal evisceration, vaginal histerectomy, laparoscopic histerectomy.

\section{Poster Session 7_Infertlity and Reproductive Medicine}

\section{P7_1}

Pelvic peritoneal adhesions - specificity of localization E.D. Dubinskaya, A.S. Gasparov, V.A. Bourlev

Department of Obstetrics, Gynaecology and Reproductive Medicine RPFU, Moscow, Russia

Summary: American Fertility Society classification (AFS) of adnexal adhesions evaluates tube-ovarian adhesions but does not describe intestinal, uterine, ligamental and other types of adhesions. The aim of the study was to evaluate endoscopic features of pelvic peritoneal adhesions (PPA).

Material \& Methods: Of 200 consecutive women with infertility and PPA laparoscopy was performed. Laparoscopy verified PPA and excluded another gynecological pathology (endometriosis, uterine myoma etc.). All the patients with PPA were divided in to two groups depending of adhesions stage according AFS classification of adnexal adhesions. The first group consisted of $67(33,5 \%)$ patients with PPA of 1-2 stage, the second - of $133(66,5 \%)$ with PPA of 3-4 stage. Endoscopic analysis was performed.

Results: In patients with stage 1-2 of PPA ovary-fossa ovarica (left $-27,4 \%$, right- $31,3 \%$ ), ovary-ligamentum latum $(20,8 \%$ left, $17,9 \%$ right) adhesions were mainly detected. The frequency of intestinal adhesions were significantly higher in patients of the second group (51,8\% compared to $8,9 \%)$. In patients of the second group peritoneal adhesions were mainly ovary-fossa ovarica (left$70,7 \%$, right $-84,2 \%$ ), ovary-uterine (left $-48,9 \%$, right $69,1 \%$ ), ovary-ligamentum latum (left- 18,8 , right $-27,7 \%$ ).

Most common were adhesions in cul-de-sac in patients of the second group compared to the first $(18,4 \%$ compared $4,5 \%)$. 
Discussion: The anatomical changes are more expressed in patients with PPA of 3-4 stages. This fact influences on patency decrease and functional possibilities of the tubes. So, the square of peritoneum included in this process is significantly higher. That proves that peritoneum reacts differently on inflammation connected hypoxia. In future, the new pathogenic mechanism of different stages and forms of pelvic adhesions would be proposed.

Key-words: pelvic peritoneal adhesions, endoscopy, infertility.

\section{P7_2}

Correlation between pelvic peritoneal adhesions stage and pelvic pain

E.D. Dubinskaya, A.S. Gasparov, V.A. Bourlev

Department of Obstetrics, Gynaecology and Reproductive Medicine RPFU, Moscow, Russia

Summary: The aim of the study was to evaluate the relationship between pelvic peritoneal adhesions (PPA) stage and severity of pelvic pain.

Material \& Methods: Of 200 consecutive women with infertility and PPA laparoscopy was performed. Laparoscopy verified PPA and excluded another gynaecological pathology (endometriosis, uterine myoma etc.). All the patients with PPA were divided in to two groups depending on adhesions stage according American Fertility Society (AFS) classification of adnexal adhesions. The first group consisted of $67(33,5 \%)$ patients with PPA of 1-2 stage, the second - of $133(66,5 \%)$ with PPA of 3-4 stage. The short form of the McGill pain questionnaire (SF-MPQ) was used for the evaluation of the severity of dysmenorrhea, CPP, and deep dyspareunia.

Results: The mean age of the patients was $32,5 \pm 0,6$ years. Total pain score significantly correlated with PPA stage. Maximal levels of SF-MPQ were registered in patients with severe pelvic adhesions and hydrosalpinx. The sensory component of dyspareunia and chronic pelvic pain according to SF-MPQ score was more expressed in patients with 3-4 stage of PPA $(p \leq 0,05)-3,94 \pm 0,26$ and $4,77 \pm 0,46$, accordingly. In case of dysmenorrheal affective component was significantly higher in patients of the second group $3,67 \pm 0,31(p \leq 0,05)$. The were no significant differences in sensory component for dysmenorrhea description among patients of both groups. At the same time the sensory component was more expressed in cases of adnexal-bowel adhesions.

Discussion: The results of the study suggest that PPA stage correlate with pain specificity. MPQ results clarify the relationship between pain intensity and PPA stage. So, the more sensory component is expressed the more severity of adhesions is. MPQ appears to be useful as a scale in description pain before surgical treatment and could be helpful as additional component for preoperative PPA stage evaluation.

Key-words: pelvic peritoneal adhesions, pelvic pain, infertility

\section{P7 3}

\section{Comparative study on dermatoglyphics in patients with pelvic peritoneal adhesions}

A.S. Gasparov, E.D. Dubinskaya O.E. Barabanova

Department of Obstetrics, Gynaecology and Reproductive Medicine RPFU, Moscow, Russia

Summary: The results of the study proved that patients with pelvic peritoneal adhesions (PPA) have some dermatoglyphic features. These changes are more expressed in patients with moderate and severe stages. These findings demonstrate that PPA are associated with genetic factors and explained the different type of peritoneum reaction on infection with or without pelvic adhesions formation.

Introduction: The aim of the study was to compare quantitative dermatoglyphic traits of patients with PPA with the control group.

Materials and Methods: 115 patients with pelvic peritoneal adhesions (PPA) were included in the study. The control group consisted of 30 fertile women. Indications for laparoscopic surgical treatment included infertility (86 $(75 \%))$ and chronic pelvic pain $(29(25 \%))$. In the control group - sterilization $(30(100 \%))$ was performed. Laparoscopy verified pelvic peritoneal adhesions and excluded another gynaecological pathology. All the patients with PPA were divided in to two groups depending on adhesion stage according AFS-classification. Dermatoglyphic analysis was performed. Through the t-test we evaluated the heterogeneity of the examined groups.

Results: The mean age of the patients was $32,5 \pm 0,6$ years. The results showed a statistically significant difference in finger ridge count cd decrease and cd distance increase in patients with PPA compared to the control group. These changes were more expressed in patients with 3-4 stage of PPA. Dermatoglyphic bilateral assimetry of finger ridge count $\mathrm{cd}$ were registered in patients with PPA $(\mathrm{p} \leq 0,05)$.

Discussion: First of all, the results proved the role of genetic factors in pelvic adhesion formation. Futhermore, dermatoglyphic analysis could be useful for preoperative PPA diagnostics.

Key-words: pelvic peritoneal adhesions, infertility, dermatoglyphic. 


\section{P7 4}

Laparoscopy in infertility: indications and findings correlation

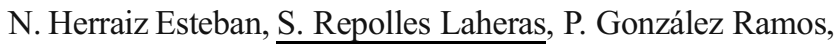
J.F. Lapresa Saenz, M. Díaz Vega

Hcu Lozano Blesa, Zaragoza, Spain

Introduction: Since 2007 laparoscopy technique was fully introduced in our hospital and we aimed to study the indications and findings in laparoscopy performed by our Infertility Section.

Materials and methods: Prospective descriptive study with a cohort population of 238 consecutive laparoscopic procedures performed in patients of our Infertility Section during the period 2006-2010. We evaluated in the sample: a) Indication of endoscope. b) Individual correlation with laparoscopic findings. T-Student for independent samples and chi-square test were used for statistical analysis.

Results: The most frequent indications of laparoscopy groups were: Abnormal HSG in 69 patients (29\%); Unexplained Infertility in 59 patients (24.8\%); Suspected endometriosis in 40 patients $(16.8 \%)$ and other factors $(28.9 \%)$ like hydrosalpinx $(\mathrm{n}=24)$, polycystic ovary syndrome $(n=17)$, adnexal tumor $(n=12)$, suspected pelvic factor $(n=10)$ and myoma $(n=6)$. Correlation: 41 patients $(17.2 \%)$ showed no evidence of genital pathology at laparoscopy. Endometriosis was found in $43 \%$ of all laparoscopies (35\% in abnormal HSG and $52 \%$ in cases of unexplained infertility with a $\mathrm{p}<0.019$ ). In the $66 \%$ of couples with unexplained infertility we found pathology related to infertility. $25 \%$ of patients had a tubal factor and $18 \%$ had adhesions in the pelvic cavity.

Discussion: Diagnostic laparoscopy is a step beyond the basic study of infertility of the couple. However, it is witnessing the rise of diseases treatable by endoscopic surgery, which can only be found by using this technique, that facilitate the resolution of sterility factor and improve the outcomes of assisted reproductive techniques.

Key-words: laparoscopy, infertility, endometriosis.

\section{P7 5}

Basal testosterone and sex hormone binding globulin levels in the prediction of stimulation parameters and cycle outcome in cycling patients undergoing in vitro fertilization

Ö. Moraloğlu, Ş. Özyer, R. Karayalçın, G. Özakşit, M. Özel, E. Tonguç, L. Mollamahmutoğlu

Zekai Tahir Burak Women's Health Education and Research Hospital, Ankara, Turkey
Objective: To evaluate basal serum testosterone (T) and sex hormone binding globulin (SHBG) levels in regularly cycling patients who undergone in vitro fertilization (IVF) treatment and their relations with stimulation parameters and IVF outcomes.

Materials and Methods: Two hundred patients seeking their first IVF treatment from June 2007 to January 2008 were evaluated prospectively. Patients aged 23-39 with regular menstrual cycles and with FSH levels less than $12 \mathrm{mIU} / \mathrm{ml}$ were included in the study. Basal concentrations of FSH, LH, E2, PRL, TSH with T and SHBG were determined. Free androgen index (FAI) was calculated. IVF stimulation parameters, fertilization and clinical pregnancy rates were evaluated.

Results: The mean age of the patients were $29.5 \pm 4.5$ years (range 23 to 39 years). The overall mean serum $\mathrm{T}$ and SHBG was $42 \pm 23 \mathrm{ng} / \mathrm{dL}$ and $42.9 \pm 27.04 \mathrm{nmol} / \mathrm{L}$ respectively. Significant positive correlation was found between $T$ and the number of oocytes retrieved and the number of embryos transferred. History of smoking was significantly associated with an increase in $\mathrm{T}$. There was a borderline significant association between fertilization failure and SHBG, being lower in patients with fertilization failure. Among the patients with cycles cancelled, $\mathrm{T}$ and FAI was found to be lower. Cycle cancellation had a borderline significant association with $\mathrm{T}$ and a significant association with FAI. However, neither T nor SHBG were significantly correlated with clinical pregnancy rates.

Discussion: Basal serum $\mathrm{T}$ levels during IVF have a positive correlation with part of the stimulation parameters including number of oocytes retrieved and the number of oocytes transferred. However, neither T nor SHBG predict fertilization or clinical pregnancy rates. Our study suggests no value to the routine measurement of T or SHBG in IVF patients in regularly cycling women without PCOS.

Key-words: testosterone, sex hormone binding globulin, in vitro fertilization.

\section{P7_6}

Conservative laparoscopic management of adnexal torsion

R. Karayalçın, S. Özcan, Ș. Özyer, T. Var, H. Yeșilyurt, H. Dumanlı, S. Soysal, L. Mollamahmutoğlu, S. Batığlu Zekai Tahir Burak Women's Health Education and Research Hospital, Ankara, Turkey

Objective: To evaluate our experience with adnexal torsion (AT) in 36 patients and the outcomes of the patients who were managed conservatively via laparoscopy.

Materials and Methods: A prospective study was conducted on 36 patients who underwent operations for AT via 
laparoscopy between January 2008 and December 2009. Data including age, previous history, the time of the onset of symptoms, the time of admission to the hospital, grayscale and color Doppler US findings, the time interval between the hospital admission and the surgery, the type of intervention, the operative findings and the postoperative gray-scale and Doppler US findings were recorded.

Results: In 29 (80.5\%) patients, a preoperative diagnosis of AT was confirmed clinically. The mean age of the patients was 26.5, with a range of 11 to 44 . Ovarian blood flow was assessed by color Doppler US ultrasonography in 30 patients preoperatively. In $11(36.6 \%)$ patients, it was found to be normal. In $19(63.3 \%)$ patients, ovarian blood flow was found to be pathological or absent. Laparoscopic conservative treatment was performed in 34 patients. In two patients, salpingo-oophorectomy was performed. No thromboembolic complications were seen. Postoperative ultrasonographic examinations confirmed normal ovarian morphology and Doppler blood flow in all patients with no recurrence.

Conclusion: Early diagnosis and treatment are key factors in managing AT. Given its demonstrated safety and benefits, a conservative approach of untwisting the adnexa and salvaging the ovary via laparoscopy should be considered in all cases of AT, regardless of the color and number of twists, in women of reproductive age.

Key-words: adnexal torsion, detorsion, laparoscopy.

\section{P7_7}

\section{Conservative surgical management of tubal and tuboovarian abscesses}

L. Mañalich, M. Bradbury, E. Suárez, T. Guerra, O. Puig, J. Xercavins

Hospital Universitari Vall d'Hebron, Spain

Introduction: Tuboovarian abscesses are most often a consequence of pelvic inflammatory disease and they are considered an important problem in reproductive medicine. For this reason, the objective of our study is to evaluate clinical and surgical outcomes depending on the surgical technique.

Material \& methods: A retrospective descriptive study (January 2000-December 2009) was performed by a review of 195 cases of severe pelvic inflammatory disease (stages III and IV), 106 of them treated surgically (71 by laparoscopic approach, 34 by open surgery and 1 by vaginal approach). The rate of intra and postoperative complications, the need of reoperation and the median postoperative hospital stay were evaluated considering the surgical technique (conservative, by means of drainage and debridement of the abscess versus radical, including the excision of the fallopian tube, ovary, adnex and/or the uterus).

Results: $32.2 \%$ of cases were performed by conservative surgery versus $68.7 \%$ of cases which were performed by excisional surgery. Considering the approach way, significant differences were found on the surgical technique (laparoscopy-39.7\% conservative versus laparotomy$18.8 \%$ conservative; $\mathrm{p}=0,042$ ). There were not statistically significant differences in the rate of operative complications, the need of reoperation and the median postoperative hospital stay depending on the surgical management.

Discussion: Considering our results and the fact that pelvic inflammatory disease affects reproductive-aged women, the conservative management for surgical treatment of the tuboovarian abscess is better than excisional surgery to try to preserve the fertility and the hormonal function.

Key-words: tuboovarian abscess, conservative surgery, excisional surgery.

\section{P7_8}

Infertile women with Premature Ovarian Failure (POF) diagnostic role of laparoscopic ovarian biopsy

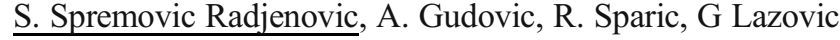
Clinic for Gynaecology and Obstetrics, Clinical Centre of Serbia, Serbia

High gonadotropins in women younger than 40 years can be accompanied by ovaries that contain follicles, but most often, the afollicular forms are seen.

The aim: 1. to analyze full thickness laparoscopic ovarian biopsies 2. To compare pregnancy rate in follicular and afollicular form of POF.

Patients and method: 40 infertile amenorrhoic women; gonadotropins and estradiol measured in two months in 4 occasions; period of study: 11 years

Results: $20 \%$ of patients had follicles in biopsy specimens. Pregnancy rate in all group is $10 \%$ : foure pregnancies and full term deliveries in ten years period. In the follicular form of POF, pregnancy rate was 35\%; in non follucular form was 3\%. Difference between the two hystologic groups in therms of pregnancy rate is significant. Two women (5\%) had hystology typical for autoimmune oophoritis, accompanied latter by adrenal autoimmunity. Thyroid autoimmunity is the most prevalent dissease accompanied to POF (in $12 \%$ of patients), followed by type 1 diabetes mellitus (2\%), but without typical hystology for autoimmune ovarian dissease. The familial form of idiopathic POF is recorded in $10 \%$ of our patients. 
Conclusion: laparoscopic ovarian biopsy is useful method to assess follicular ovarian reserve and possibilities for the reproduction. It gives the useful data for the investigation of etiopathogenesis of POF: autoimmunity, familial appearance and genes involved in etiology of the disease.

Key-words: POF, laparoscopy, ovarian biopsy.

\section{P7_9}

\section{Failure of the conservative treatment of ectopic pregnancy. Results of Cruces Hospital}

I. Davalillo, M.A. Arribalzaga, J.S. Alonso, I. Brouard Urkiaga,

S. Díez Lázaro

Cruces Hospital, Spain

Summary: A total of 120 cases of ectopic pregnancies with conservative treatment (expectant attitude or methotrexate) have been analyzed in order to identify the failure of the first, between April, 2004 and April, 2010 in the Hospital of Cruces. The global rate of failure was 13,4\% and these cases were solved successfully by means of surgery.

Introduction: Inside the management of ectopic pregnancy, the possibility of conservative treatment exists, whether it's with methotrexate or expectant attitude. These patients follow a strict control. In the cases in which the conservative treatment turns out to be ineffective rescue surgery is necessary.

Materials and Methods: Our objective is to analyze the failures of conservative treatment of the ectopic gestation. There are 120 registered cases of ectopic pregnancies, 93 (77.5\%) of which were treated with methotrexate and in 27 cases $(22.5 \%)$ was decided expectant attitude. We have analyzed pre-treatment $\beta-\mathrm{HCG}$ levels, doses of methotrexate, number of doses administered, success and failure rates, average time of resolution and associated complications in all cases.

Results: Of the 93 the cases treated with methotrexate, 35 $(37.6 \%)$ received $50 \mathrm{mg}$ and $58(62.3 \%) 75 \mathrm{mg}$ i.m. The success of the medical management was of $86.6 \%$ (104/ 120). The surgical indication was established in 16 cases. The average time of resolution was of 32 days and the associated complications were the following: pain (3 cases), gastrointestinal symptoms ( 3 cases) and one case of hematosalpinx.

Discussion: Thanks to its lower morbimortality, efficacy, safety and minor cost, the medical treatment of the ectopic pregnancy is the therapeutic option of choice, in cases adequately selected, under strict control.

Key-words: ectopic pregnancy, methotrexate.
P7 10

Follow-up of laparoscopic treatment in hydrosalpinx. A clinical study of 99 cases

C. David, I.A. Lupascu, D. Socolov, R. Socolov

University of Medicine and Pharmacy Gr T Popa- Iasi, Romania

Introduction: Hydrosalpinx is a frequent cause for tubal infertility, which can be easily diagnosed and treated by laparoscopy. In this study we evaluate the results of laparoscopic treatment in this type of pathology.

Materials and methods: We studied 99 cases of distal tubal infertility, diagnosed by ultrasound, hysterosalpingography, sono-hysterography with contrast and/or laparoscopy. The tubal score was assessed according to French scoring system proposed by Mage et al. All cases were submitted to laparoscopy and different procedures performed: adhesiolysis, fimbrioplasty, neosalpingostomy and dye-test. The follow-up included a 3 month hysterosalpingography to evaluate tubal patency.

Results: The results after 3 months showed 78.8\% tubal patency at hystero-salpingography. From these $47.4 \%$ had pregnancies in 6-9 months, and $16.7 \%$ ectopic pregnancies. Conclusions: The conservative laparoscopic treatment in distal tubal pathology is adequate if proper selection of cases using an international scoring system, and can significantly improve fertility.

Key-words: tubal infertility, laparoscopy, hydrosalpinx.

\section{Poster Session 8_Innovation in Surgery}

\section{P8_1}

Laparoscopic surgery of intact ovarian dermoid cysts A. Kavallaris, S. Mytas, N. Chalvatzas, A. Hornemann, K. Diedrich

University of Schleswig-Holstein, Campus Luebeck, Germany

Introduction; Dermoid cysts are common in young women. Cystectomy via laparotomy was performed as a first choice therapeutic procedure until the 1990ies, but more recently laparoscopy has replaced laparotomy. Intraperitoneal spillage of dermoid cyst content, if not followed immediately by abundant peritoneal lavage, can cause chemical peritonitis with subsequent adhesion formation. To date, only few small case series on the effectiveness and complications of laparoscopic cyst removal have been reported. Herein we report a large case series in which a new technique of placing the dermoid cyst above an endobag during 
enucleation has been implemented in order to avoid peritonitis in case of spillage.

Study objective: To evaluate the incidence of spillage and recurrence rate of laparoscopic ovarian dermoid cystectomy. Patients and methods: A retrospective evaluation of operations performed over a 7-year period (September 1999January 2006) . 121 premenopausal women with dermoid cysts (mean diameter on sonography: $6.5 \pm 1.50 \mathrm{~cm}$, range 3 to $12 \mathrm{~cm})$ were treated by laparoscopy. We assessed the duration of the surgical procedure, the incidence of spillage and complications, the length of hospitalization, and the incidence of recurrences and pregnancies.

Results; In 15/121 (12. 4\%) cases the cyst ruptured during enucleation, however, obvious spillage of endocystic contents occurred in only $3 / 121(2.5 \%)$ patients in which the endobag ruptured during removal. A second operation was performed in 35 of the 121 patients; in 9 (7, 4\%) patients because of dermoid cysts on the contraleteral ovary, in $4(3,3 \%)$ patients because of recurrence. Nine (7, 4\%) patients experienced spontaneous pregnancy within the observational period. In one patient ovarial strumal carcinoid (a distinctive tumor composed of thyroid tissue and carcinoid), and in another patient an immature teratoma of the ovary was diagnosed. No signs or symptoms of peritonitis were observed in all women regardless of cystic spillage occuring or not.

Discussion: dermoid cyst with a diameter from $3-12 \mathrm{~cm}$ can effectively be removed by laparoscopy with a low ipsilateral recurrence rate. A spillage rate of $12 \%$ was observed in this case series, and the patients need to be counselled about this potential complication. However, controlled intraperitoneal spillage of cyst contents does not increase postoperative morbidity as long as the peritoneal cavity is thoroughly washed out and most likely because a technique of performing the operation above an endobag was employed. Laparoscopic conservative cystectomy of dermoid cysts in premenopausal women is safe and effective and appears to be a valuable alternative to laparotomy. Key-words: ovarian dermoid cyst, laparoscopy, recurrences and pregnancies.

\section{P8 3}

\section{Uterine arterial embolization with embozene for treatment of uterine fibroids}

C.M. Martin Diaz, A. Pinas Carrillo, M.I. Diaz-Plaza Perez, M.D. Maldonado Del Valle, C. Lanciego Perez, M.L. Cañete Palomo

Hospital Virgen de la Salud, Toledo, Spain

Introduction: Uterine arterial embolization is considered a minimally invasive, safe and efective alternative treatment to hysterectomy, myomectomy or hormone therapy in women suffering from sintomatic uterine fibroids. The aim of our study is to compare the efficacy of "EMBOZONE", a new embolizating particle, in contrast to the traditional ones.

Materiasl and methods: We designed a prospective cohort study. It included 156 patients from December 2002 to June 2009. First 112 patients were embolized with the traditional polyvinyl alcohol foam (PVA) (December 2002- October 2007), whilst the next 44 patients were treated with the new Embozone microspheres (October 2007-June 2009). For both groups of treatment we determined Haemoglobin, Haematocrit, and the presence of menorrhagia and/or dysmenorrhoea before and after the procedure.

Results: Both study groups presented similar characteristics at the start of the study. We observed an improvement in the clinical manifestations in both groups (controlled bleeding in $91 \%$ of cases and improvement on the dysmenorrhoea in $92 \%$ among the traditional particles group whilst $87 \%$ and 96\% respectively in the Embozone group). Both groups also experimented an improvement in the degree of anaemia.

Discussion: According to the data obtained, embolization with EMBOZONE microspheres offers similar results to the traditional PVA and could be used as a first treatment as it minimizes the surgical risk and improves postprocedure recuperation. Still, we need more studies to establish these new microspheres as a first choice treatment in uterine arterial embolization.

Key-words: fibroid, embolization, embozene.

\section{P8_4}

\section{Clinical outcomes of uterine fibroid embolization after five years}

A. Piñas Carrillo, C.M. Martín Diaz, M.N. Rodriguez Martín, M.D. Maldonado Del Valle, L. García-García, M.L. Cañete Palomo

Hospital Virgen De La Salud, Spain

Summary: Uterine fibroid embolization (UFE) is a non surgical treatment option for symptomatic patients who wish to avoid surgery. We performed an observational retrospective study including 40 patients. UFE continues to be a safe and effective alternative treatment for symptomatic uterine fibroids five years after the procedure.

Introduction: Uterine fibroid embolization (UFE) is based upon the hypothesis that bilateral reduction of uterine arteries blood flow will result in infarction of fibroids and therefore, control symptoms. The aim of our study is to review the clinical outcomes after a five year follow-up in our hospital. 
Material and method: We performed an observational retrospective study. It included 40 patients who had completed five years of monitoring. We determined Haemoglobin, Haematocrit, and the presence of amenorrhoea, menorrhagia and/or dysmenorrhoea five years after the procedure.

Results: The mean age of our patients at the time of the procedure was 42 years old. The data obtained showed an improvement in the dysmenorrhoea and controlled bleeding subsequently decreasing the anaemia, in $100 \%$ cases going through uterine fibroid embolization. The rate of permanent amenorrhoea post UFE in women after five years was $35 \%$.

Discussion: UFE continues to be a safe and effective treatment five years after the procedure. Permanent amenorrhoea is considered a major complication in patients below 40 years, and a minor complication over 40 . However, if we consider the mean age for menopause, the natural onset of this event during the clinical follow-up could act as a confounding factor in the outcomes.

Key-words: embolization, uterine fibroids, menorrhagia.

\section{P8_5}

Minimal access approach in the management of acute abdomen of gynaecologic origin

L. Ples, M. Burtea, C. Ignat, S. Paun

Bucur Maternity, St Ioan Clinical Emergency Hospital, Bucharest, *Clinical Emergency Hospital Bucharest, Romania

Background: Nowadays laparoscopy has become an accepted method of management for gynaecologic emergencies. The aim of this study is to determine the benefits of laparoscopy in diagnosis and treatment of acute abdomen of gynaecologic origin.

Material and Methods: A retrospective study was carried out in 54 patients with acute abdominal pain of suspected gynaecologic origin, who underwent emergency laparoscopic approach in the last 2 years.

Results: There were 40 (74.1\%) complicated ovarian cysts (OC) and $14(25.9 \%)$ ectopic pregnancies (EP) with tubal rupture. The mean age was $27.9 \pm 6.12$. 26(65\%) OC were located on the right ovary. EP were equally located on the left and right side. There were $10(72 \%)$ ampulary EP. Ultrasound sensibility in hemoperitoneum diagnosis was $80 \%(\mathrm{OC}) / 100 \%(\mathrm{EP})$ and $85 \%(\mathrm{OC}) / 71 \%(\mathrm{EP})$ in visceral lesions. Time admission-OR was $15.78 \pm 21.2$ hours. We performed intraoperative haemostasis in $4 \mathrm{OC}(10 \%), 36$ cystectomies $(90 \%), 10$ salpingectomies $(71.4 \%)$ and 4 (28.6\%) tube-preserving interventions. 3 patients from EP group needed blood transfusion $(\mathrm{P}=.008$, Cramer's
$\mathrm{V}=0.59)$. Discharge after 87 hours $(\mathrm{OC}) / 124$ hours(EP) $(\mathrm{P}=.046)$. There was no mortality.

Conclusions: Laparoscopy is a useful diagnostic and therapeutic tool for acute abdomen of gynaecologic origin. EP with tubal rupture in emergency conditions had a powerful correlation with blood transfusion.

Key-words: gynaecologic emergency, laparoscopic approach, hemoperitoneum.

\section{Poster Session 9_Myomectomy}

\section{P9 1}

Pregnancy and complication rate after laparoscopic myomectomy sutured with single stitches in only one layer

A. Kavallaris, N. Chalvatzas, C. Banz, K. Diedrich, A. Hornemann

University of Schleswig-Holstein, Campus Luebeck, Germany

Introduction: Myomectomy is a common laparoscopic procedure and is often used in patients with hypo fertility, bleeding disorders and other symptoms caused by leiomyomas.

Patients and Methods: We present a case series report based on a retrospective audit conducted from January 2001 up to December 2006 in our department. From 451 patients laparoscopically operated for leiomyomas, we identified only 59 patients operated due to hypo fertility reasons. We report the postoperative rates of pregnancy and mode of delivery after a median follow up to 40 months postoperatively. Laparoscopic technique and obstetrical outcome is discussed with recent literature review.

Results: The average number of removed fibroids was at least 2. The mean weight of the leiomyomas was $94.3 \mathrm{~g}$. The cavum uteri were opened in 8 patients. We found a conception rate after laparoscopic myomectomy of $78 \%$ with a total of 60 pregnancies in 46 patients. The miscarriage rate postoperatively was 8 out of 60 pregnancies $(13 \%)$. Finally 42 patients have 51 deliveries of live births took place giving a life birth rate postoperatively of $71 \%$.

Discussion: In patients with leiomyomas identified as infertility cofactor, laparoscopic management is a convincing therapeutic approach. Conceiving rates are high and morbidity during pregnancy ranks within normal limits. However we observed a risk for uterine rupture during labor $(4 \%)$, therefore mode of delivery should be discussed in detail with affected patients.

Key-words: myomectomy, laparoscopy, follow up. 


\section{P9 2}

\section{Long-term results of outpatient hysteroscopic myomectomy: our experience A. Lebre, A.R. Pinto, M.I. Sá, M. Leal Centro Hospitalar do Porto, Portugal}

Summary: A retrospective study was performed of all the hysteroscopic myomectomies realized in our outpatient department during a 7-year period. Several data were analysed and we concluded that outpatient hysteroscopic myomectomy has a very low rate of complications and excellent long-term outcome, when an adequate selection of patients and proper surgical skills are used.

Introduction: Submucous myomas are frequently associated to menorrhagia and infertility. A minimally invasive procedure such as bipolar outpatient hysteroscopic myomectomy seems to have a low complication rate and good long-term results.

Materials and Methods: A retrospective study was performed of all the hysteroscopic myomectomies realized in our outpatient clinic during a 7-year period. Patient's symptoms, characteristics of the fibroids, rate of complications, follow-up, residual myoma, persistence of symptoms and pregnancy rate were analysed.

Results: 114 patients were submitted to hysteroscopic myomectomy in our outpatient department during this 7year period. $53.5 \%$ had menorrhagia as their main symptom. According to the Wamsteker classification $80.7 \%$ were type 0 myomas; their mean size was $17.8 \mathrm{~mm}(6-41 \mathrm{~mm})$. No relevant intraoperative complications were registered and mean follow-up was 15 months (3-45 months). One-step excision was achieved in $88.6 \%$ of patients, while 8 patients needed a second hysteroscopic myomectomy and 5 were posteriorly submitted to hysterectomy due to persistence of symptoms.

Discussion: Our data suggests that outpatient hysteroscopic myomectomy has a very low rate of complications and excellent long-term outcome, when an adequate selection of patients and proper surgical skills are used.

Key-words: myomectomy, long-term, hysteroscopy.

\section{P9 3}

\section{Myomectomy or uterine artery embolisation for uterine fibroids - where's the evidence? \\ J. Daniels, FEMME Trial Collaborative Group \\ University of Birmingham, UK}

Introduction: Uterine fibroids are the most common tumour in women of reproductive age and increase in prevalence with age. We updated the Cochrane meta-analysis with three randomised trials comparing uterine artery embolisation (UAE) with hysterectomy. A difference in terms of patient satisfaction (OR $0.6 ; 95 \% \mathrm{CI} 0.2-1.4 ; \mathrm{p}=0.2$ ) was not seen, but hysterectomy reduced reintervention rates (OR 3.2; 95\%CI 1.4-7.1; $\mathrm{p}=0.004$ ). For those not wishing to lose their uterus, the choice lies between myomectomy and UAE. Rationale One small $(n=121)$ single centred randomised clinical trial compared myomectomy with UAE for intramural fibroids. Both approaches appear to improve quality of life, but with little randomised data for these very different options. Since the options are so different, requiring very different hospital stays with very different outcomes including fertility, sexual function and recurrence, a fair and comprehensive comparison is both overdue and complex. Trial Protocol The FEMME Trial is a multicentre randomised trial funded by the UK NIHR Health Technology Assessment Programme. 650 women with symptomatic fibroids wishing to retain fertility potential will be randomised to myomectomy or UAE. The primary outcome of quality of life will be assessed by use of a disease specific questionnaire UFS-QOL at two years. Effectiveness will also be assessed at 6 months and 1 and 4 years after treatment. Secondary outcomes include effect on menstrual bleeding, pregnancy outcomes, further treatment and adverse events. Ovarian function and reserve will be assessed on a sub-group of women. Data on resource use will be collected to allow an economic evaluation, to be conducted concurrently. Recruitment will commence in Autumn 2010.

Key-words: randomised controlled trial, uterine artery embolisation, myomectomy.

\section{P9_4}

Comparison of effect and pain relief between intravenous fentanyl citrate and interval analgesia after laparoscopic myomectomy

K. Ito, H. Asada, H. Tsuji, M. Furuya, Y. Yoshimura, I. Kishi, K. Kobiri

Keio University of Medicine, Department of Obstetrics and Gynaecology, Japan

Objective: To compare the effectiveness of intravenous fentanyl citrate and interval analgesia with pentazocine intramuscularly after laparoscopic myomectomy in the reduction of postoperative pain and adverse events.

Methods: Fifteen patients were recruited afeter laparoscopic myomectomy. Postoperatively ten patients received intravenous fentanyl citrate and droperidol ; five were infused with fentanyl citrate $20.0 \mu \mathrm{g}$ h-1 (IV group A), the rest five patients were infused with fentanyl citrate $13.3 \mu \mathrm{g}$ h-1 (IV group B), and five patients were prescribed pentazocine $30 \mathrm{mg}$ and hydroxyzine hydrochloride $25 \mathrm{mg}$ intramuscu- 
larly (IM group). Postoperative pain was evaluated at 1, 2, 8 and 12 hours after surgery using a verbal rating scale (VRS). Adverse events were evaluated with nausea, vomiting and activities of daily living (ADLs).

Result: The VRS relief rating scores for pain did not significantly differ in three groups, but in each group the VRS relief rating scores were decreased with duration. Pentazocine IM group resulted in higher pain scores than the fentanyl IV groups; total of VRS relief rating scores was 8 in IV group A, 12 in IV group B and 17 in IM group. Control of postoperative nausea and vomiting were inclined to be better in IV groups than IM group ( $33 \%$ vs $80 \% ; \mathrm{P}=0.125)$. No difference in ADLs was found in the three groups.

Conclusion: Intravenous fentanyl citrate may be superior to interval analgesia with pentazocine intramuscularly for the pain relief, nausea and vomiting after laparoscopic myomectomy. A large-scale study will be needed to confirm these analgesic effects.

Key-words: postoperative pain, laparoscopic surgery, verbal rating scale.

\section{P9_5}

\section{Using a patch of collagen coated with fibrinogen and thrombin (Tachosil®) in laparoscopic myomectomy: a small number for a new approach to surgical hemostasis}

C. Sommella, F. Lelli, A. Mezzesimi, A. Joghtapour, L. Alamanni, G. Arcamone

U.O. Ginecologia ed Ostetricia, Ospedale S. Maria alla Gruccia Montevarchi (AR), Italy

Introduction: In this paper we describe our first cases of laparoscopic myomectomy in which it was used for haemostasis control a patch of collagen coated with fibrinogen and thrombin (Tachosil ${ }^{(\mathbb{R})}$ ) The surgical control of bleeding is a very important factor in the course of laparoscopic myomectomy. To prevent bleeding, especially in case of intramural myomas of considerable size, it was proposed to pretreat patients with $\mathrm{GnRH}$ agonists, or administered during surgery substances such as oxytocin or vasopressin. None of these safeguards is still as important as surgical skill.

Material \& Methods: The five patients studied were all with no prior problems such coagulation (examinations with preoperative values of PT, PTT and antithrombin III in normal). The patients underwent surgery for the presence of intramural myomas. Regarding medical history there are no special disease worthy of note, only one of them had a previous surgery for laparoscopic appendectomy. The surgical technique is briefly summarized. The patient is placed in lithotomic position. You insert a uterine mobilizer and a urinary catheter. We run a cord access by open technique under vision with optical trocar. $\mathrm{CO} 2$ is insufflated to obtain pneumoperitoneum and placed two other ancillary trocar in the left and right iliac region of five and ten millimeters. Receive the myoma is incised through the serosa and myometrium by monopolar hook with pure cutting current to reduce thermal damage to surrounding tissues and promote good repair. Identified the pseudocapsule of the myoma that is excised and traction maneuvers with lysis of adhesions pseudocapsule trying to avoid using current coagulation. This fact allows for a good repair of the myometrium. The uterus breach is then sutured with interrupted sutures with absorbable thread with intracorporeal or extracorporeal knot. Alternatively the wires can be used that do not require locking type of nodes. This is followed by the morcellation of myomata and suture of the abdominal. In the series under consideration following the breach of the uterine suture, to achieve perfect hemostasis, we used a patch of collagen coated with fibrinogen and thrombin $\left(\right.$ Tachosil $\left.^{\circledR}\right)$. For introduction into the cavity of the support was used technique we designed "double arrow" that allowed the rapid introduction of the support and the ease of attaching the breach of the same uterus.

Results: In the series under consideration following the breach of the uterine suture, to achieve perfect hemostasis, we used a patch of collagen coated with fibrinogen and thrombin (Tachosil $\left.{ }^{\circledR}\right)$. For introduction into the cavity of the support was used technique we designed "double arrow" that allowed the rapid introduction of the support and the ease of attaching the breach of the same uterus. The average duration of surgery was $73 \pm 15$ minutes and mean hospital stay of $2 \pm$ 1 days. No postoperative complication was recorded and the average loss in hemoglobin between the preoperative and postoperative examinations was $1.7 \pm 0.6 \mathrm{~g} / \mathrm{dL}$.

Discussion: The Tachosil ${ }^{\circledR}$ has led to a rapid and complete haemostasis of the uterine gap also makes the surface of bloody serous insulated from the surrounding organs. This phenomenon could be exploited to prevent or reduce adhesions that often form in such places precisely because of gemizi blood. Obviously, further studies will be needed to substantiate this hypothesis. It will be particularly useful follow-up of patients who have undergone this type of myomectomy in case of re intervention for other conditions. On this occasion it will be possible to assess the presence or absence of adhesions at the previous uterine scar.

Key-words: miomectomy, laparocopy, tachosil.

\section{P9 6}

Dissecting leiomyomas of the uterus - one case report E. Xia

Hysteroscopic Center,Fuxing Hospital, Capital Medical University, China 
Summary: Dissecting leiomyoma naked eye looked like a vicious, but a benign histology and clinical course. There is fragmentation of the growth pattern. Due to its rich blood supply, a tumor nodule grew faster, and extends to the growth of distance, but showed benign after clinical attention. It should be pay attention to avoid misdiagnosis and over-treatment of malignant. Introduction Dissecting leiomyoma of the uterus was rare and bizarre form which may give rise to mistaken for malignant tumors although it's benign.

Material \& Methods: 32 years old woman, myomectomy 17 months after surgery were detected in the pelvic mass. Uterus liked 8 weeks and $10 \times 8 \times 7 \mathrm{~cm}$ size of its left mass which closed to the uterus. B ultrasonography shown posterior uterus, in which left side an irregular hypoechoic mass about $7.7 \times 7.0 \times 5.5 \mathrm{~cm}$ was found, nodular internal echo color Doppler could be detected and its rich blood flow signals. Results: On 2009-3-2 hysterectomy, both salpingo- oophorectomy and the left pelvic mass excision were performed. The left tumor size is about $10 \times 7 \times 4 \mathrm{~cm}$, tumor extended to the pelvic floor up to the left levator ani muscle, weight 200 grams. Pathological diagnosis: Dissecting leiomyoma. Followed up for 12 months after operation without recurrence and metastasis.

Discussion: Dissecting leiomyoma is a rare variant of benign leiomyoma that is closely related to and shares some features with other and similarly named leiomyoma variants. It should also be distinguished from other unusual leiomyoma variants, especially to exclude intravascular spread, for appropriate classification and potential prognostic implications.

Key-words: dissecting leiomyoma, uterus, pathological diagnosis.

\section{P9_7}

The effect of the learning curve and the surgeon's experience on the surgical outcome of laparoscopic myomectomies

C. Ros, M.A. Martínez-Zamora, F. Carmona, C. CasteloBranco, J.A. Vanrell, J. Balasch

Institut Clínic de Ginecologia, Obstetrícia i Neonatologia. Hospital Clínic de Barcelona. University of Barcelona, Spain

Summary: A learning curve is demonstrated in the conservative laparoscopic management of myomas with a shorter operating time, with no change in other surgical parameters. Introduction: We assessed the effect of the increasing surgeon's experience and the learning curve on the surgical outcome of laparoscopic myomectomies.

Methods: A retrospective observational study including 280 patients who underwent a laparoscopic myomectomy in a tertiary referral centre (Group 1: 140 first laparoscopic myomectomies and Group 2: 140 last laparoscopic myomectomies). Myoma extraction was performed by using an electric morcellator.

Results: Mean number of myomas: Group 1: $1.7 \pm 1.1$; Group 2: $1.8 \pm 1.8(\mathrm{p}=0.3)$. Mean size of the biggest myoma (mm): Group 1: 67.2 \pm 21 ; Group 2: $80.8 \pm 23$ ( $<<0.0001)$. Mean size of the sum of all myomas (mm): Group 1: 79.9 31; Group 2: $101.5 \pm 46(\mathrm{p}<0.0001)$. The mean duration of surgery (minutes) was: Group 1: 178 \pm 70 ; Group 2: $147 \pm$ $56(\mathrm{p}<0.0001)$. There were 11 conversions to laparotomy in Group 1 and 13 in Group $2(\mathrm{p}=0.3)$. Only one bladder lesion was reported during the surgery in Group 2. Postoperative complications including febrile syndromes, haemoperitoneums that required subsequent surgery and hysterorrhaphy haematomas that did not require more surgery were reported in 10 patients in Group 1 and 9 patients in Group $2(\mathrm{p}=0.6)$. There were no differences in post-operative haemoglobin levels $(\mathrm{p}=0.7)$ : Group 1: $9.9 \pm$ $1.4 \mathrm{~g} / \mathrm{dl}$; Group 2: $9.7 \pm 1.7 \mathrm{~g} / \mathrm{dl}$. Inpatient stay postoperatively was similar in both groups (days): Group 1: 2.6 \pm 1.3 ; Group 2: $2.8 \pm 1.5(\mathrm{p}=0.5)$.

Discussion: A learning curve is demonstrated in the conservative laparoscopic management of myomas with a shorter operating time, with no change in other surgical parameters. Furthermore, with increasing surgeon's experience, there was a significant increase in the size of myomas operated.

Key-words: laparoscopic myomectomy, learning curve, surgical outcome.

\section{P9_8}

\section{Who should be submitted to hysteroscopic myomectomy?}

A. Lebre, A.R. Pinto, M. I. Sá, M. Leal

Centro Hospitalar do Porto, Porto, Portugal

Summary: A retrospective study was performed of all the hysteroscopic myomectomies realized in our outpatient department during a 7-year period. Patient's age, parity, menopausal status, symptoms and myoma size and location were analyzed. We concluded that hysteroscopic resection remains the gold standard for the treatment of submucous myomas in most of the patients.

Introduction: Surgical management of submucous fibroids has changed in the last decades from laparotomy to minimally invasive surgery, and it depends on the patient's desire to conceive, symptoms, location and size of fibroids.

Materials and Methods: A retrospective study was performed of all the hysteroscopic myomectomies realized in 
our outpatient clinic during a 7-year period. Patient's age, parity, menopausal status, symptoms, and location and size of fibroids were analyzed.

Results: 114 patients were submitted to hysteroscopic myomectomy in our outpatient department during this 7year period. Their mean age was 48 years $(21-75$ y) and mean parity was 1.5 children ( $0-4$ children). $41 \%$ of the patients were in the menopause and $53.5 \%$ had menorrhagia as their main symptom. According to the Wamsteker classification $80.7 \%$ were type 0 myomas; myoma's mean size was $17.8 \mathrm{~mm}(6-41 \mathrm{~mm})$. One-step excision was achieved in $88.6 \%$ of patients.

Discussion: Our data suggests that hysteroscopic resection remains the gold standard for the treatment of submucous myomas in most of the patients.

Key-words: myomectomy, hysteroscopy, outpatient.

\section{Poster Session 10_Office \& Diagnostic Hysteroscopy}

\section{P10_1}

\section{Novasure impedance controlled endometrial ablation- Shorter treatment times are likely to be associated with increased pain scores in the outpatient setting \\ P. Balchandra, S. Jones \\ Bradford Royal Infirmary, UK}

Treatment times in outpatient NovaSure impedance controlled endometrial ablation vary between $40-120$ seconds, (Mean 88 seconds). Our retrospective audit aimed to determine characteristics, pain scores, complications and treatment outcome of patients undergoing outpatient NovaSure at our hysteroscopy clinic between 2006-2009 based on treatment times of 120 seconds or more( $1 \mathrm{st}$ group) and 60 seconds or less (2nd group). Of the 87 patients in total, 1st group had $7(8 \%)$ while 2 nd group had $10(11 \%)$ patients. $57 \%$ had spontaneous vaginal deliveries (SVD), 14\% LSCS and 28\% were nulliparous in 1 st group while $100 \%$ had SVD's in 2 nd group. $28 \%$ had a small fibroid on hysteroscopy in the 1st group. All had normal hysteroscopies in 2nd group. In the 1st group, ultrasound(USS) was not done in $71 \%$, was normal in $14 \%$ and multiple intramural fibroids were found in $14 \%$. No patients had USS in 2nd group. Mean VAS pain score during procedure was 4 in 1st group and 6 in 2nd group. $42 \%$ in 1 st group had minor complications whilst no complications in 2nd group. 14\% likelihood of Rollerball ablation in 1 st group and $10 \%$ in 2nd group Amenorrhoea rates at 3,6 and 12 months were $28 \%, 57 \%$ and $57 \%$ in 1 st group and $20 \%, 30 \%$ and $30 \%$ in the 2 nd group. $60 \%$ failed to follow up at 12 months in 2 nd group. To conclude , amenorrhoea rates were better however complications were more likely with an increased need for further procedures in the 1st group compared to the 2 nd group. Shorter treatment times were more likely to be associated with increased pain scores.

Key-words: novasure, endometrial ablation, amenorrhoea.

\section{P10 2}

Utility of Bettocchi system in office hysteroscopy

G. Germano, A. Achard, M. Hermida, A. Rubal, G. Antunez, A. Saldías

Hospital Pereira Rossell, Uruguay

Objective: To evaluate the usefulness of the Bettocchi System in the diary practice of office hysteroscopy.

Methods: Analysis of the data base of the Centro Regional de Endoscopía Ginecológica Del Hospital Pereira Rossell, Montevideo,from May 2005 to April 2010. In this period we preformed 6863 vagina- hysteroscopy, using the Bettocchi System, distension with saline solution, and without use of speculum, neither tenaculum.

Results: We used the grasping or/and scissors in 1138 cases, ( $16,4 \%$ ); in the other 5725 cases it wasn't necessary. The mean indication in these cases was IUD extraction, in 58\%; followed by abnormal bleeding in 20,5\%, and ESSURE collocation in $4 \%$. We made 677 IUD extractions, 137 polipectomies, 176 adhesiolisis and Intern orifice openings; and 50 ESSURE collocations. The polyps extracted measured between 0,5 and $3 \mathrm{~cm}$, most of them $(80 \%) 1$, $5-2 \mathrm{~cm}$. The use of cervical anesthesia was needed in $3 \%$ (28 patients).

Conclussion: The systematic use of the Bettocchi System in the diary office hysteroscopy facilitates the approach to the uterine cavity thanks to its ovoid section; reduce de pain in patients, and the need of anesthesia, and permit to make directed biopsies and polipectomies in the same time we are making the diagnosis of pathology. In addition, gives the possibility of making a permanent sterilization by ESSURE. Key-words: Bettocchi system, office hysteroscopy, polipectomy.

\section{P10 3}

The first extra-hospitalary hysteroscopy unit in Barcelona. Results of the first year in ambulatory Essure

S. Haimovich, C. Serra, E. Del Amo, G. Mancebo, R. Carreras

Hospital del Mar de Barcelona. IMAS, IMIM, Spain 
Objective: Review of the ESSURE procedures performed in a Public Primary Health Center between January 2009 and January 2010

Methods: 120 ESSURE insertions cases were evaluated prospectively by questionnaire. We asked the patients to evaluate the information quality, pain and satisfaction.

Results: $70 \%$ of our patients were immigrants and only $30 \%$ local population. The mean value of age was 39 (range 25-47) and the mean value of parity was 2,6 (range 0-6). Procedure was performed without any kind of anesthesia with a Bettocchi hysteroscop of 4,3 $\mathrm{mm}$. No speculum or tenaculum were used, just vaginoscopy. Correct procedure en 114 (95\%) of the cases. No insertion due to anatomical difficulties or tubal spasm in 6 $(5 \%)$ cases. Mean value of the procedure's duration was of 4,5 minutes (range 3-8). Pain and satisfaction was evaluated. We had only 3 cases of very light vagal syndromes. $85 \%$ of the patients returned to normal life immediately.

Conclusion: - ESSURE insertion in an extrahospitalary environment is highly accepted by the patients with low pain and high satisfaction - This is a safe technique that may be performed outside the hospital. - It has advantages for the patients and for the hospitals due to reduction of the assistencial pressure.

Key-words: ESSURE, office hysteroscopy, vaginoscopy.

\section{P10_4}

\section{Malignant potential of endometrial polyps}

G.E. Cano, M.P. Guillén, J.E. Arjona Berral, E. Velasco Sanchez, J.J. Serrano Dávalos

Hospital Universitario Reina Sofia, Cordoba, Spain

Objectives: To determine the pre-malignant and malignant potencial of endometrial polyps, and to asses whether different clinical parameters are associated with malignancy in the polyps.

Materiasl and methods: 1492 hysteroscopic resections of endometrial polyps were reviewed. Histological diagnosis and clinical characteristics (presence of abnormal uterine bleeding and polyps size) were analyzed. Statiscal analysis was performed.

Results: In our study included 1492 patients, 576 premenopausal and 916 postmenopausal women. The main indication for hysteroscopy was abnormal uterine bleeding, which was $60,8 \%$ in the premenopausal group and $55 \%$ in the postmenopausal group. The most common ultrasound diagnosis was suspected thickened endometrial and suspected polyp (78\%). The histology of the majority of polyps were benign $(75 \%$ hyperplasia without atypia and $12 \%$ atrophic postmenopausal). There were 11 cases of hyperplasia with atypia $(0.73 \%), 10$ cases in postmenopausal women and a 1 case in a premenopausal woman. There were 9 cases of endometrial carcinoma on polyp (0.62\%), 8 cases in postmenopausal women and as a single case in premenopausal women, most of them with symptoms of menorrhagia. Of the patients included in the study, 66 Treatment with tamoxifen, and only one showed endometrial cancer. In $90 \%$ of cases resection of the polyp was performed by outpatient hysteroscopy. We also analyzed the size of the polyp in conjunction with histology.

Conclusiones: The likelihood of having a cancer on endometrial polyp is $0.62 \%$ in our study population. This probability increases in symptomatic menopausal patients. In premenopausal women without symptoms who have an endometrial polyp $<15 \mathrm{~mm}$ and without any risk factors, may obviate the removal of the polyp and conducted follow-up.

Key word: endometrial polyps.

\section{P10_5}

\section{Office hysteroscopy for the removal of intrauterine} device

R. Nonell, M. Martinez Terron, M. Cardona, P. Jou Icgon Hospital Clinic Barcelona, Spain

Summary: Retrospective study based on 110 patients with failure of IUD removal and subsequent office hysteroscopyc procedure: $92.6 \%$ successful retrieval of the IUD, good tolerance, no major complications. Introduction: Intrauterine device (IUD) is a widely accepted method of contraception. Blind removal of missing IUD strings may be traumatising and unsuccessful.

Objectives: Evaluate the feasibility of hysteroscopy to confirm the diagnosis and guide removal of the missed IUD.

Materiasl and Methods: 110 patients with failure of IUD removal were sending to Hysteroscopy Service from January 2001 to December 2009. Office hysteroscopy was performed with 4.5 or 5.5 continuous flow hysteroscope. The Items studied were: diagnostic hysteroscopy, successful rate of retrieval, patient's tolerance, complications and need for further acts.

Results: Median age: 40.4 years (21-65). 11\% menopausal. 19\% Chinese IUD 15 patients diagnostic hysteroscopy only: 7 empty cavities (2 laparoscopic extractions by IUD migrate into peritoneal cavity). 2 IUD perforating uterine wall (laparoscopic extraction).2 IUD norm inserts and 4 removals with forceps. Of the reminder 95 patients, 88 (92.6\%) had successful removal of the device in an office setting: $66(75 \%)$ without anaesthesia and 25\% with Para cervical anaesthesia, without complications and good tolerance ( $74 \%$ pain $\leq 5$ in VAS). In 7 patients IUD must be retrieved by hysteroscopy in the operating room, with 1 auto limited haemorrhagic episode. 
Discussion: Hysteroscopy by direct visualization of the uterine cavity will enable gynaecologists to provide optimal care to the patients with missing IUD strings.

Key-words: migrate IUD, office hysteroscopy, removal.

\section{P10 6}

Uterine polyps: prevalence, symptoms, pathology and treatment preferences as part of the outpatient polyp treatment (OPT) trial in a nurse-led outpatient hysteroscopy clinic

S. O'Connor, L. Gennard, N.A.M. Cooper, T.J. Clark

Birmingham Women's Hospital / University of Birmingham, UK

Introduction: Endometrial polyps are a common problem in gynaecology but management varies widely. Endometrial polyps are the focus of the outpatient polyp treatment (OPT) trial, which aims to establish whether removal of polyps in the outpatient setting is as efficient as removal as an inpatient under general anaesthetic when women present with abnormal uterine bleeding (post-menopausal, intermenstrual or heavy menstrual bleeding). Symptomatic women are randomised unless they express a preference for treatment setting. This multicentre trial is based at Birmingham Women's Hospital where in addition to consultant led clinics a successful nurse-led outpatient hysteroscopy clinic has been running since February 2008. Materials and Methods: prospective electronic database of standardised clinical data as part of the OPT trial within a nurse-led outpatient hysteroscopy clinic.

Results: From February 2008 until June 2010, 309 patients were seen in the nurse-led hysteroscopy clinic and 71 $(22 \%)$ of these women were diagnosed with endometrial polyps. $65(92 \%)$ women presented with PMB, and $6(8 \%)$ were asymptomatic Two women did not have their polyps removed; one because she was due to undergo vaginal hysterectomy and the second declined further investigation and treatment. Of the remaining 69 women 42 agreed to take part in the OPT trial, 26 were randomised between outpatient and inpatient removal, 16 entered the preference arm of the study with 12 having a preference for outpatient treatment and 4 for inpatient. We have so far obtained histology reports for 59 patients (we need to assess the other 10 further). $66.1 \%$ of the polyps were reported as benign, $27.1 \%$ as hyperplasia, $1.7 \%$ as malignant and $5.1 \%$ of the samples were non-diagnostic.

Discussion: In postmenopausal women endometrial polyps are prevalent ( 1 in 5) and associated with abnormal bleeding. The majority of polyps are benign although at least 1 in 4 is hyperplastic, supporting current practice of polypectomy. Women appear to have a preference for outpatient treatment and the OPT trial will help establish the effectiveness and acceptability of outpatient management.

Key-words: nurse hysteroscopist, endometrial polyp, postmenopausal bleeding.

\section{P10 7}

Changes in clinical practice after the implementation of an office hysteroscopy unit

A. Rodriguez Oliver, J. Fernández Parra, A. Gonzalez Paredes, I. Vico, F. Montoya

Servicio de Obstetricia y Ginecologia. Hospital Virgen de las Nieves, Granada, Spain

Office hysteroscopy became the primary approach for the diagnosis and management of most of the uterine intracavitary patology performing in a single act. Only selected patients may be treated in an operating room setting.

Introduction: The purpose of this study was to analyze the repercussions of the establishment of an outpatient hysteroscopic unit on the habitual management of operating room hysteroscopy.

Materials and methods: A descriptive research about office and operating room hysteroscopic techniques in the last years was done. Furthermore, we reviewed and analyzed their complications and evolution in the time in function of the introduction of new technologies and the endoscopic experience.

Results: Four thousand twenty six outpatient hysteroscopies and one thousand one hundred-three operating room hysteroscopies were analyzed. The failure rate was similar in both groups (2.5 versus $2.6 \%$ respectively); whereas the intra-operative complications were very different. The pain and the vaso-vagal syndrome predominated in outpatient hysteroscopy; however, the perforations and cervical lesions were more frequent in the inpatient setting. As the experience about hysteroscopy has increased, more surgical tecniques as polypectomies and directed biopsy have been performed. The operating room procedures have decreased significantly and the myomectomy has became the main indication in the last years.

Key-words: office hysteroscopy, inpatient hysteroscopy, complications.

\section{P10_8}

Hystologic findings in patients with vaginal bleeding I. Romero Nieto, J.J. Serrano Dávalos, G. Exojo Cano, J.E. Arjona Berral, E. Velasco Sánchez, Servicio de Obstetricia y Ginecología del Hospital Universitario Reina Sofia, Córdoba, Spain 
Objective: To determine the histopathological findings in patients with metrorrhagia who underwent an ambulatory hysteroscopy at Hospital Universitario Reina Sofia from Córdoba .

Material and methods: We performed a retrospective study including all women with vaginal bleeding who underwent ambulatory hysteroscopy between July 2005 and December 2009.

Results: From a total of 3518 hysteroscopies, 1651were performed in menopausic women, in which the results were the following: $732(44,33 \%)$ had endometrial atrophy; 462 $(27,98 \%)$ endometrial polyps; $127(7,69 \%)$ endometrial cancer; $115(6,96 \%)$ normal endometrium; 60 (3,63\%) endometrial hyperplasia without atypia; $26(1,57 \%)$ myomas; 24 (1,45\%) endometrial hyperplasia with atypia; 23 $(1,39 \%)$ insufficient sample; y $82(5 \%)$ had other hystologic results. In the premenopause group, from a total of 1867 hysteroscopic procedures, the results were the following: 1108 (59.35\%) had a normal endometrium, 299 (16.02\%) endometrial polyps, $196(10.5 \%)$ endometrial hyperplasia without atypia, 141 (7,56\%) myomas; 50 (2.68\%) endometrial atrophy, 22 (1.17\%) insufficient sample, 9 (0.48\%) Endometrial cancer, $1(0.05 \%)$ endometrial hyperplasia with atypia and $41(2.19 \%)$ had other results.

Conclusion: In both groups $(44.33 \%$ and $59.35 \%)$ the principal histological finding was a normal endometrium. Thus, hysteroscopic procedures in women with vaginal bleeding should be reserved for cases in which anomalies are detected through a prior vaginal ultrasound All hystologic alterations, including endometrial cancer were more frequent in menopausic women. The most common hystologic alteration found in both groups were benign endometrial polyps.

Key-words: histopathological findings, metrorrhagia, ambulatory hysteroscopy.

\section{P10_9}

\section{The incidence of endometrial carcinoma in atypical hyperplasia \\ I. Romero Nieto, G. Exojo Cano, J.E. Arjona Berral,} M. Pola Guillén, I. Ilic

Servicio de Obstetricia y Ginecología del Hospital Universitario Reina Sofia, Córdoba, Spain

Objective: This study was conducted to evaluate the association between atypical endometrial hyperplasia and endometrial carcinoma in women whose preoperative histeroscopic byopsy were reported as atypical hyperplasia. To evaluate the stage and the grade of the tumors associated to atypical hyperplasia. And to evaluated the hormonal status of tha patients.

Materials and methods: Endometrial biopsies were performed by hysteroscopy in the Department of Gynaecology and Obstetrics of the Reina Sofía Hospital, for the period from July 2005 through December 2009.

Results: Of the entire group of 385 patients with endometrial hyperplasia, 9,61\% (37 women) had atypical hyperplasia, and the vast majority of patients had simple hyperplasia $(84,68 \%)$. Among de 37 patients who were diagnosed prior to hysterectomy as atypical hyperplasia, 70,27\% had endometrial carcinoma. According to the grade and stage, 3 endometrial cancers were in situ-stage $0(11,53 \%) ; 5$ cases $(19,25 \%)$ were well differentiated-stage Ia; $11(42,3 \%)$ were well differentiated-Ib; 4 (15,39\%) were well differentiated invasive-Ic; and $3(11,53 \%)$ were moderate differentiated-IV. Most of the endometrial cancers were diagnosed in menopausal patientes $(92,30 \%)$. Among patients who had a preoperative tissue diagnosis of atypical hyperplasia, 8 of 37 $(33,33 \%)$ who underwent a hysterectomy had both atypical hyperplasia and endometrial carcinoma in their uterus.

Conclusion: $70,27 \%$ of the patients who were diagnosed as atypical hyperplasia, in fact, had endometrial carcinoma, and different stages including advanced carcinoma. At this institution, atypical hyperplasia should be treated with a total hysterectomy. Therefore, an adecuated diagnosis should be made before surgery. Additionally, an intraoperatory biopsy must be performed in order to determine the tumor stage. Only the $33,33 \%$ associated atypical hyperplasia and endometrial cancer.

Key-words: atypical endometrial hyperplasia, endometrial carcinoma, hysterectomy.

\section{P10_10}

Hysteroscopy as an outpatient setting. Our 5 year experience

J.J. Serrano Dávalos, I. Romero Nieto, J.E. Arjona Berral, G. Exojo Cano, J.A. Monserrat Jordán

Servicio de Obstetricia y Ginecología del Hospital Universitario Reina Sofia, Córdoba, Spain

Objective: Determine the chief complaint, hysteroscopic findings and histological results in patients who underwent an ambulatory hysteroscopy.

Materials and methods: We performed a retrospective study including all women who underwent ambulatory hysteroscopy between July 2005 and December 2009.

Results: A total of 5955 hysteroscopic procedures were performed, of which 3309 (55.56\%) were done in preme- 
nopause and 2644 (44.44\%) in postmenopause women. Chief Complaint.- In the premenopause group, the chief complaint in 1867 cases $(56.43 \%)$ was vaginal bleeding, in 524 cases $(15.84 \%)$ were abnormal ultrasound findings (endometrium $\geq 15 \mathrm{~mm}$ ) and 216 cases $(6.53 \%)$ were derived for sterility. On the other hand, in menopausic women, 1651 (62.41\%) were derived for vaginal bleeding, $628(23.75 \%)$ for abnormal ultrasound findings and 155 (5.87\%) for endometrial follow-up due to tamoxiphen treatment. Hysteroscopic Findings.- In the premenopause group, 1148 women $(43.76 \%)$ had a normal endometrium and $1024(30.95 \%)$ had endometrial polyps. The results in the menopause group were as follows: 873 women $(33.1 \%)$ had endometrial atrophy and in 637cases $(24.08 \%)$ an endometrial polyp was discovered. Histologic Findings.Histologic findings in premenopause women were the following: 1993 women $(60.22 \%)$ had no hystologic alterations, and $575(17.36 \%)$ were benign endometrial polyps. In contrast, 1109 (42\%) of postmenopause women had endometrial atrophy, $872(32.96 \%)$ had benigng endometrial polyps, $153(5.79 \%)$ were diagnosed of endometrial cancer and in 30 cases $(1.13 \%)$ endometrial hyperplasia with atypia was found.

Conclusion: In both groups the chief complaint was metrorraghia, followed by anormal ultrasound findings. Also, in both groups the most common hysteroscopic finding was the lack of endometrial disease, followed by endometrial polyps. Histology confirmed the lack of endometrial disease in $63,02 \%$ of premenopause and in $47.86 \%$ of the menopause group. Incidence of endometrial disease confirmed by histology is higher in the menopause group.

Key-words: chief complaint, hysteroscopic findings, histologic findings.

\section{P10 11}

\section{Long term contraceptive efficacy of Essure device}

E. Velasco Sánchez, J.E. Ríos Castillo, J.E. Arjona Berral, B. Povedano Cañizares, J.A. Monserrat Jordán Hospital Universitario Reina Sofía, Córdoba, Spain

Objectives: To assess the contraceptive efficacy of Essure device five years after placement. To compare its contraceptive efficacy with other definitive methods.

Materials and Methods: Retrospective study of 1200 women who underwent hysteroscopic tubal sterilization with Essure device after five years follow up. All data are recollected in the database of the Office Hysteroscopy Unit of the Hospital Universitario Reina Sofía. We compared our results with the results of other definitive methods published in the CREST study.

Results: In the 1200 patients that have completed five years follow up, we have registered three cases of pregnancies. In two cases, the women were pregnant before procedure. The other pregnancy occurred during the three months waiting period, while she was using oral contraceptive and the migration of one micro insert was probed. That means a failure rate of $0,25 \%$ even that the real rate it's $0,083 \%$ if we only take into account pregnancies after procedure. The results of CREST study after five years of procedures: bipolar tubal ligation:1,65\%; monopolar: $0,23 \%$; ring: $1 \%$; Hulka clip:3,17\%; elective salpinguectomy: $1,5 \%$. Total of methods: $1,3 \%$.

Conclusions: The permanent birth control Essure device is one of the most effective definitive methods in avoiding pregnancies, maintaining its effectiveness after five years of placement. Key-words: pregnacy, definitive method, Essure.

\section{P10_12}

\section{Outpatient management of endometrial polyps in our Hospital: Hospital Universitario Reina Sofía}

G. Exojo Cano, J.J. Serrano Dávalos, I. Romero Nieto, J.E. Arjona Berral, E. Velasco Sánchez, J.E. Monserrat Jordan

Hospital Universitario Reina Sofia, Córdoba, Spain

Objectives: To assess the outpatient management of endometrial polyps in our Hysteroscopy Unit. To evaluate the efficacy and the benefits obtained for the hospital.

Material and Methods: Retrospective cohort study of 1447 patients with the hysteroscopic diagnosis of endometrial polyps of our unit, from July 2005 till December 2009. In all of them, polyp resection with micro scissors or versapoint was attempted. We analyzed the hormonal status, symptoms, histological findings and treatment received.

Results: $61.5 \%$ (889) of women were postmenopausal. The most frequent symptom was metrorrhagia and because of ultrasound findings for both groups. The histology of polyps was: hyperplasia $75 \%$, atrophic $12 \%$, complex hyperplasia $3.04 \%$, with atypia $0.62 \%$, with cancer $0.62 \%$. We have performed 1342 outpatient polypectomies $(90 \%)$, 269 every year. $60 \%$ were resected with versapoint and $40 \%$ with micro scissors. Only in $10 \%$ of cases was performed an operative hysteroscopy; we have saved 54 days of operation room every year. The estimated cost per year for outpatient polypectomy is $74.683 €$, and it would be $269.000 €$ for operative hysteroscopy. 
Conclusions: Most of polyps were diagnosed in postmenopausal women with abnormal bleeding, with a low incidence of malignity. With the use of versapoint, most of polyps can be treated in an outpatient basis; that allows a better and efficiency management of operation rooms and hospitalary stances. In our hospital, outpatient management of polyps saves $194000 €$. That makes versapoint a costeffective instrument.

Key-words: office hysteroscopy, endometrial polyps, cost-effectiveness.

\section{P10 13}

\section{Hysteroscopic (Essure ${ }^{\circledR}$ ) versus laparoscopic sterilisation: demography and women's preferences in a nurse-led outpatient clinic}

T. Bingham, N. Cooper, T.J. Clark

Birmingham Women's Hospital, UK

Background: Laparoscopic sterilisation has been the standard method for women requiring permanent contraception. However, more convenient, office-based hysteroscopic sterilisation using the Essure ${ }^{\circledR}$ permanent birth control system has become established over the last 5 years. With the introduction of the Essure ${ }^{\circledR}$ technology, our experience was that the choice of sterilisation method was strongly influenced by the prejudices of the consulting gynaecologist. In order to minimise this bias, we established a standardised, independent nurse-led clinic to see all referrals for female sterilisation from primary care.

Methods: Prospective data for all women referred to our nurse-led clinic for female sterilisation between December 2007-2009 to the Birmingham Women's Hospital (University Teaching Hospital in a large urban area) were entered into an electronic database which was interrogated to examine women's characteristics and preferences for type of contraceptive method.

Results: 376 women were seen in the two-year study period. $113 / 376$ (29\%) women changed their mind and decided to use an alternate reversible contraceptive method with most selecting the Mirena ${ }^{\circledR}$ intrauterine device (101/376, 27\%). Of the 263 women undergoing sterilisation, significantly more women had a preference for Essure ${ }^{\circledR}$ hysteroscopic sterilisation compared with laparoscopic sterilisation (148/263, $56 \%$ vs. $115 / 263,44 \%, \mathrm{P}=0.004)$. There were no significant differences between the mean age (36), BMI (27), parity (3) of women in relation to choice of sterilisation method. Past caesarean section did not influence sterilisation choice (prevalence $21 \%$ in both groups).The prevalence of men- strual problems $(103 / 376,27 \%)$ did not increase the uptake of the MirenaTM $(27 / 103,26 \%$ vs. $74 / 273,27 \%$; $\mathrm{P}=0.9)$.

Conclusion: Office-based hysteroscopic Essure ${ }^{\circledR}$ sterilisation is chosen by at least half of all women seeking permanent birth control. Preference does not appear to be influenced by age, BMI, parity or prevalence of caesarean section. Almost one in three women requesting sterilisation in primary care may decide to use the Mirena ${ }^{\circledR}$ device following comprehensive counselling, although this choice does not seem to driven by the presence of problematic menstrual periods. Comprehensive, non-biased counselling is essential to allow women to make an appropriate informed choice as to contraceptive method and type of sterilisation.

Key-words: Essure, hystersocopic sterilization, laparoscopic sterilization.

\section{P10_14}

Office hysteroscopy in the diagnosis of postmenopausal bleeding

C. Costa, A. Melo, M. Martinho

Gynaecological Endoscopy Unit, Department of Obstetrics and Gynaecology - S. João Hospital, Porto, Portugal

Introduction: We aimed to study hysteroscopic and histopathologic findings in cases of postmenopausal bleeding.

Materials and Methods: Review of all office hysteroscopies performed in postmenopausal patients with uterine bleeding over a year in a tertiary care hospital. Ultrasound and hysteroscopic findings, histopathology and management were assessed.

Results: Seventy one cases of postmenopausal bleeding were identified (13.9\% of all performed hysteroscopies). In 43 cases $(60.6 \%)$ there was a diffuse endometrial thickening and in 11 cases (15.5\%) an endometrial polyp was suspected on ultrasound; in 16 cases $(22.5 \%)$ there was no reference to ultrasound findings. On hysteroscopy, a benign endometrial polyp was the most common finding $(\mathrm{n}=28 ; 39.4 \%)$, followed by suspected malignancy $(\mathrm{n}=13 ; 18.3 \%)$ and endometrial atrophy $(\mathrm{n}=$ 9; 12.7\%). Histopathology was available in 51 cases. Simple endometrial hyperplasia without atypia was the most common histopathologic diagnosis $(n=15 ; 21.1 \%)$, followed by endometrial polyp with no other specification $(\mathrm{n}=12 ; 16.9 \%)$; endometrial cancer was found in 11 cases $(15.5 \%)$. Most of the speciems for hystologic examination were obtained through endometrial biopsy during hysteroscopy $(\mathrm{n}=28 ; 54.9 \%)$; in $15(29.4 \%)$ patients it was obtained through office polypectomy with mechanical scissors. 
Discussion: The diagnosis of malignancy in this subset of patients was far more common when compared with the total population (3.7\% of all hysteroscopies performed over the assessed year), and most of the histologic specimens were obtained during office hysteroscopy. Therefore hysteroscopy plays an important role in the assessment of postmenopausal bleeding.

Key-words: postmenopausal bleeding, endometrial cancer, office hysteroscopy.

\section{P10 15}

\section{Office hysteroscopy: clinical and histopatologic characterization of endometrial polyps identified over a year in a tertiary care unit \\ C. Costa, A. Melo, M. Martinho \\ Gynaecological Endoscopy Unit, Department of Obstetrics and Gynaecology - S. João Hospital, Porto, Portugal}

Introduction: We aimed to characterize endometrial polyps identified through office hysteroscopy.

Materiasl and Methods: Review of all office hysteroscopies performed over a year in a tertiary care hospital, in which histology confirmed an endometrial polyp. Menopausal status, abnormal uterine bleeding, reason for referral for hysteroscopy, hysteroscopic findings, histopathology and management were assessed.

Results: Two hundred and one polyps were confirmed by histology; 123 patients were postmenopausal, and 66 had abnormal uterine bleeding. Ninety three cases (46.3\%) were referred for suspected endometrial polyps, and 88 (43.8\%) for diffuse endometrial thickening on ultrasound. Benign endometrial polyp was the most common finding on hysteroscopy $(\mathrm{n}=174 ; 86.6 \%)$. Simple endometrial hyperplasia without atypia was the most common histopathologic diagnosis $(\mathrm{n}=90 ; 44.8 \%)$, followed by endometrial polyp with no other specification $(n=76 ; 37.8 \%)$. Complex endometrial hyperplasia with atypia, and endometrial cancer were found in 5 cases $(2,5 \%)$, all in postmenopausal women, but only one of them had abnormal uterine bleeding. In most cases, office polypectomy with mechanical scissors was performed $(n=112$; $55.7 \%)$; in 46 cases $(22.9 \%)$ polypectomy with unipolar resectoscope was done.

Discussion: Most endometrial polyps were benign, both in pre and postmenopausal women, with and without abnormal uterine bleeding. However, not all cases of malignancy were suspected on hysteroscopy, and only one was associated with abnormal uterine bleeding, though all were diagnosed in postmenopausal women. Therefore it may be advisable to remove any identified endometrial polyp especially in postmenopausal women.

Key-words: endometrial polyps, office hysteroscopy, histopathology.

\section{P10 16}

\section{Radiological follow-up following Essure ${ }^{\circledR}$ sterilisation:} TVS or HSG?

P. Jain, T. Bingham, N. Cooper, T.J. Clark

Birmingham Women's Hospital, UK

Background: The safety, effectiveness and convenience of hysteroscopic Essure ${ }^{\circledR}$ sterilisation has resulted in it fast becoming the female method of choice for permanent birth control. Hysterosalpingogram (HSG) has been the standard method to confirm adequacy of sterilisation, but the test is relatively invasive and may deter some women from the Essure $^{\circledR}$ method. Pelvic ultrasound (TVS) is less invasive and can be used to determine the correct location of the Essure $^{\circledR}$ microinserts in uncomplicated procedures. We aimed to evaluate TVS to see if had the potential to reduce the use of HSG at 3 months post Essure ${ }^{\circledR}$ sterilisation. Materials and Methods: Standardized prospective data for all women undergoing hysteroscopic Essure ${ }^{\circledR}$ sterilisation between December 2007-2009 to the Birmingham Women's Hospital (University Teaching Hospital in a large urban area) were entered prospectively into an electronic database. A standardized protocol was introduced to strictly define an uncomplicated Essure ${ }^{\circledR}$ procedure suitable for follow up with TVS. The proportions of women undergoing TVS and/or HSG were determined.

Results: 150 women underwent hysteroscopic Essure ${ }^{\circledR}$ sterilisation of which 133 (89\%) were considered uncomplicated and were allocated radiological follow-up with TVS, of which 115 complied. TVS confirmed correct placement in $96 / 115$ cases (85\%). Of the inconclusive cases on TVS, all underwent HSG that confirmed bilateral tubal occlusion. 16/17 (94\%) of the procedures considered complicated had bilateral tubal occlusion confirmed. One woman had a tubal perforation detected at laparoscopy.

Discussion: TVS can be used as the radiological method of choice for confirming adequacy of hysteroscopic Essure ${ }^{\circledR}$ sterilisation in the majority of uncomplicated procedures. HSG should be restricted to procedures considered difficult or where TVS is equivocal. Longer term data are needed to determine the accuracy of TVS compared with HSG in predicting tubal occlusion following hysteroscopic Essure ${ }^{\circledR}$ sterilisation.

Key-words: Essure, hysteroscopic sterilization, transvaginal ultrasound. 


\section{P10 17}

\section{Operative office hysteroscopy}

P. Lobo Abascal, J. Álvarez Bernardi, J. Rubio Valtueña, J. Heras Aznar, C. García de Santiago

Hospital Infanta Sofia. San Sebastián de los Reyes, Madrid, Spain

Introduction: Office hysteroscopy, together with endometrial biopsy, is considered the gold standard for the investigation of abnormal uterine bleeding and other conditions involving the uterine cavity. Technological advances, together with "not touch approach", have contributed to improve the results of operative office hysteroscopy with good tolerance and satisfaction.

Materials and methods: Retrospective descriptive analysis of 976 office hysteroscopies performed between May 2008 and May 2010 in our Center.

Results: $97,54 \%$ of the procedures were performed in outpatient setting. The main indications were: Premeopausal Patients: indications $n=530$. Evaluation of the anomalous uterine bleeding: 362, 68,3\%. Diagnosis and treatment of suspected intrauterine pathology: 115, 21,7\%. Investigation of infertility, diagnosis and treatment of uterine MF: 16,3\%. Lost intrauterine devices: 27, 5,1\%. Other: 10, 1,9\%. Postmeopausal Patients: indications $\mathrm{n}=$ 446. Evaluation of the anomalous uterine bleeding: 274 $.61,4 \%$. Diagnosis and treatment of suspected intrauterine pathology: 111, 24,9\%. Increase of the endometrial thickness in asymptomatic patients: 56 12,6\%. Other: $5,1,1 \%$.

$93,4 \%$ of the pathology was resolved during the office Hysteroscopy, including polyps $\geq 3 \mathrm{~cm}$, myomas $<2 \mathrm{~cm}$ and uterine septums. Only $6,4 \%$ of patients needed surgical hysteroscopy under anaesthesia. The procedure was acceptable and well tolerated in $87 \%$ of the patients. Only $3,2 \%$ of the patients experienced severe pain during the hysteroscopy. No major complications were found in our series.

Discussion: The use of and an atraumatic insertion technique, bipolar electrode and mini-hysteroscopes along with the use of premedication and good technical skills is very important to resolve most of the endometrial pathology in outpatient setting.

Key-words: operative hysteroscopy, office hysteroscopy.

\section{P10_18}

Incidence and management of endometrial polyps in women with post-menopausal PV bleeding (PMB) in the office setting

W. MacNab, T. Majumudar, H. Abdel-Rahman

Hinchingbrooke Healthcare NHS Trust, UK
Summary: 1 in 4 women presenting to our 'one-stop PMB clinic' had endometrial polyps and $95 \%$ of these were benign. Two-thirds of these were safe and effectively removed in the office setting. Introduction: To assess the incidence of polyps in women with PMB and to evaluate the need for and efficacy of office hysteroscopic polypectomy.

Materials and Methods: Prospective study of 160 women with uterus presenting with PMB between 11.11.08 to 10.2.10

Results: $38.8 \%$ women with an endometrial thickness (ET) $<5 \mathrm{~mm}$ on ultrasound were reassured and discharged without further investigations. Of the remaining, $98 \%$ had a hysteroscopy with $82.3 \%$ having outpatient office hysteroscopy at the same visit. $25 \%$ women with PMB had polyps and in those with an ET $>5 \mathrm{~mm}$ the incidence was $41.7 \%$. $95 \%$ of these were benign. $68 \%$ had office hysteroscopic polypectomy at the first visit.

Discussion: Endometrial polyps are common in women presenting with PMB. Most of these are benign. Office hysteroscopic polypectomy can be performed safely and effectively in these women in a one-stop clinic. The role of routine polypectomy in the diagnosis of endometrial cancer remains unclear and needs further evaluation as most polyps are benign and sensitivity of a pipelle biopsy by itself in the diagnosis of endometrial cancer is very high (98\%).

Key-words: endometrial polyps, office hysteroscopy, polypectomy.

\section{P10_19}

\section{Hysteroscopic sterilization with Essure: 4 years of experience}

S. Maia, A.R. Abreu, C. Moreira, M. Oliveira Hospital Infante Pedro EPE, Aveiro, Portugal.

Introduction: Hysteroscopic sterilization, performed by the bilateral placement of the micro-insert Essure into fallopian tube lumens, is a safe and effective method of permanent contraception. Tubal occlusion and proper positioning of the micro-inserts should be confirmed 3 months after the placement. The aim of this study was to evaluate our experience with Essure tubal sterilization and review the follow-up visit performed at 3 months.

Materials and Methods: Retrospective analysis of medical records of 250 patients who underwent hysteroscopic sterilization using Essure between February 2006 and March 2010 was performed. Parameters studied include age, parity, successful bilateral micro-insert placement rate, complications, tubal occlusion based on pelvic radiography and/or hysterosalpingography (HSG), follow-up rate and pregnancies reported. 
Results: Initially the procedures were performed under general anaesthesia in an operating room. After most women underwent placement of Essure in outpatient setting with only oral ibuprofen and achieved a bilateral micro-insert placement at the first attempt. Patients who presented a satisfactory placement, underwent pelvic radiography after 3 months to confirm the position and alignment of the micro-inserts. HSG was performed in the cases of unsatisfactory placement in order to confirm bilateral tubal occlusion. There was one pregnancy reported secondary to misinterpreted HSG.

Discussion: The patient should be instructed to return for the follow-up visit. Pelvic radiography appears to be a reliable confirmatory test in cases of satisfactory placement. Essure tubal sterilization is a feasible hysteroscopic procedure in the outpatient setting.

Key-words: Essure, hysteroscopic sterilization.

\section{P10_20}

\section{Introduction of Hysteroscopic tubal sterilization technique Essure in Hospital Infanta Sofia}

P. Lobo Abascal, J. Álvarez Bernardi, J. Rubio Valtueña, S. Duch Grau, A. Garicano

Hospital Infanta Sofía. San Sebastián de los Reyes, Madrid, Spain.

Introduction: We analyze the results of the first 73 ESSURE devices inserted in our Center in outpatient setting.

Materials and Methods: Retrospective descriptive study of 73 patients in which the insertion of the ESSURE devices in office setting was performed between June 2009 and March 2010.

Results: The mean age of the patients was 36,9. In 95,8 \% of the patients insertion of both devices was achieved in the first attempt. In the $97,2 \%$ of them the procedure was consider to be of low or medium difficulty. Tolerance was good in $91 \%$ of the patients and mild in $8,21 \%$ The average insertion time was 6.7 minutes. 3 months after the insertion an image test was performed to verify the correct insertion of the devices. In 7 patients $(9,5 \%)$ hysterosalpingography was indicated due to difficult or no reassuring insertions. In 6 of these cases occlusion of both tubes was confirmed. In the rest of the patients control were made with pelvic X-ray and transvaginal ultrasonography. Only one laparoscopic tubal sterilization has been indicated after the control. To date no pregnancies have been reported.

Discussion: We believe that the results obtained in the first 73 cases of ESSURE hysteroscopic permanent sterilization procedure have been satisfactory and are similar to those reported by other groups. Wide experience in office hysteroscopy is important to achieve successful ESSURE insertion but some special technical considerations may be kept in mind ESSURE hysteroscopic permanent sterilization procedure is safe, well tolerated and provides high rate of satisfaction in patients with a minimum percentage of complications.

Key-words: office hysteroscopy, tubal sterilization.

\section{P10 21}

Essure Implants for Tubal Sterilisation in France. Hysteroscopic tubal sterilisation: french multicentric cohort study SUCCES II

P. Panel ${ }^{1}$, S. Heckel ${ }^{2}$, J.B. Engrand ${ }^{3}$, R. Hsiung ${ }^{4}$, A. Agostini $^{5}$, V. Villefranque ${ }^{6}$, R. Kutnaorsky ${ }^{7}$, P. Lopes ${ }^{8}$, H. Martigny ${ }^{9}$, F. Marchand ${ }^{9}$, C. Chis $^{10}$, J. Thevenot ${ }^{11}$, C. Dhainault ${ }^{12}$

${ }^{1} \mathrm{CH}$ de Versailles, 78150 Le Chesnay. ${ }^{2}$ Hopital Saint Joseph 69000 Lyon. ${ }^{3}$ Maternité des Bazennes, 59431 Saint Paul sur Mer. ${ }^{4}$ Polyclinique de Franche-Compté ${ }^{5}$ CHU La Conception 13000 Marseille ${ }^{6} \mathrm{CH}$ René Dubos, 95300 Pontoise. ${ }^{7}$ Centre de la Mère et de l'Enfant, 68024 Colmar ${ }^{8}$ CHU Nantes, 49093 Nantes Cedex. ${ }^{9}$ Polyclinique de l'Atlantique, 45819 Saint Herblain Cedex. ${ }^{10} \mathrm{CHU}$ Bichat, 75018 Paris. ${ }^{11}$ Clinique Ambroise Paré, 31083 Toulouse Cedex 1. ${ }^{12}$ Hôpital Privé Nord Parisien, 95200 Sarcelles

The aim of this cohort study was to evaluate feasibility and acceptability of Essure ${ }^{\circledR}$ placement without anesthesia. This multicentre study covered 11 French facilities from September 2008 through March 2010 and collected information about anaesthesia, analgesics, pain, uterine cavity, visibility of the ostia and placement outcome. This study included 1148 attempted placements of Essure ${ }^{\circledR}$ micro-insert. In 4 cases, procedure didn't occur because of lack of visibility. The procedure was done with no anaesthesia in $87 \%$ of cases. Before procedure, analgesics was given to $86,2 \%$ of patients (NSAID: $31,6 \%$; combination with NSAID: $37,1 \%$; non NSAID: $17,5 \%$ ). Uterus was retroverted in $21 \%$ of cases. Both ostia were visualised in $93 \%$ of cases. In 1063 cases (93\%), the first placement attempt was successful. VAS pain measurement at the time of hysteroscope introduction in the cervix was $\leq 3$ for $73 \%$ and $\geq 8$ for $8 \%$ of women. VAS pain measurement at the time of implant placement was $\leq 3$ for $59 \%$ and $\geq 8$ for $10 \%$ of women. Predictive factors for pain were past tubal surgery, PID, endometriosis, no use of analgesic or NSAID alone. Predictive factors for failure were lack of ostia visualisation, retroverted uterus and pain. $97 \%$ of women were satisfied; unsatisfaction was correlated with pain and placement failure. More inclusions in this cohort study could help to determine subgroups where 
procedure should not be attempted or where analgesia should be required.

Key word: Essure.

\section{P10 22}

Essure implants for Tubal Sterilisation in France. Control of placement at three months: about 666 cases P. Panel $^{1}$, S. Heckel ${ }^{2}$, J.B. Engrand ${ }^{3}$, R. Hsiung ${ }^{4}$, A. Agostini ${ }^{5}$, V. Villefranque ${ }^{6}$, R. Kutnaorsky ${ }^{7}$, P. Lopes ${ }^{8}$, H. Martigny 9 , F. Marchand ${ }^{9}$, C. Chis ${ }^{10}$, J. Thevenot ${ }^{11}$, C. Dhainault ${ }^{12}$

${ }^{1} \mathrm{CH}$ de Versailles, 78150 Le Chesnay. ${ }^{2}$ Hopital Saint Joseph 69000 Lyon. ${ }^{3}$ Maternité des Bazennes, 59431 Saint Paul sur Mer. ${ }^{4}$ Polyclinique de Franche-Compté ${ }^{5}$ CHU La Conception 13000 Marseille ${ }^{6} \mathrm{CH}$ René Dubos, 95300 Pontoise. ${ }^{7}$ Centre de la Mère et de l'Enfant, 68024 Colmar ${ }^{8}$ CHU Nantes, 49093 Nantes Cedex. ${ }^{9}$ Polyclinique de l'Atlantique, 45819 Saint Herblain Cedex. ${ }^{10} \mathrm{CHU}$ Bichat, 75018 Paris. ${ }^{11}$ Clinique Ambroise Paré, 31083 Toulouse Cedex 1. ${ }^{12}$ Hôpital Privé Nord Parisien, 95200 Sarcelles

This is part of a French multicentre cohort study about hysteroscopic tubal sterilisation conducted in 11 facilities from September 2008 through March 2010. The aim of this study was to analyse modalities and results of control at three months of hysteroscopic tubal sterilisation by Essure ${ }^{\circledR}$. Standard control protocol is based on $\mathrm{X}$ ray for normal insert placement procedure or hysterosalpingography (HSG) in case of difficulties during Essure ${ }^{\circledR}$ placement. When $\mathrm{X}$ ray didn't reach acceptable criteria, HSG was recommended. This study included 1148 attempted placements of the Essure ${ }^{\circledR}$ microinsert. 666 patients had control at three month and could be analysed. Standard X ray was performed in 529 cases $(79 \%)$ and HSG in 97 cases (14.5\%). Nevertheless, ultrasound was performed alone in 79 cases $(11.8 \%)$ and associated with $\mathrm{X}$ ray for 334 women (50\%). With respect of protocol and in absence of ultrasound, HSG should have been performed in 33\% of cases. When $\mathrm{X}$ ray was correct, twice ultrasound showed a wrong placement of implants; in an other hand, when $\mathrm{X}$ ray didn't meet criteria, ultrasound was sufficient to confirm good placement in two third of the cases. Finally, physicians concluded to a good result in 649 cases $(97.4 \%) ; 2$ patients presented an implant's expulsion and 8 patients a implant's perforation or migration.

Analysis of those data showed that if ultrasound was systematically associated with standard X ray, HSG should be required in only $11 \%$ of cases $(7 \%$ of normal placements and $27.5 \%$ of difficult placements).

Key word: Essure
P10 23 Correlation between ultrasound scan and hysteroscopy
in the diagnosis of metrorrhagia

M. Andrés Arribalzaga, M. Goitia Ibarra, I. Brouard Urkiaga, I. Davalillo Bilbao, J.S. Alonso, I. Vázquez Lerma Cruces Hospital, Bilbao,Spain

Introduction: In the diagnostic study of metrorrhagia the hysteroscopy plays a basic role, since it allows the direct visualization of the uterine cavity and the possibility to perform directed biopsy. The ultrasound scan orientates the diagnosis, but if there's a suspicion of endometrial pathology we must resort to the hysteroscopy for its confirmation and, if possible, its treatment.

Materials and Methods: A total of 2,073 cases of hysteroscopy in the Hospital of Cruces between November, 2007 and June, 2010 have been analyzed in order to observe the findings in pre- or postmenopausal metrorrhagias, and the correlation between them and the ultrasound scan study.

Results: 781 (37.67\%) of diagnostic hysteroscopys were realized due to metrorrhagia, premenopausal in 370 of the cases and postmenopausal in the remaining 411 cases. In the group of postmenopausal metrorrhagia, the findings in the ultrasound scan were: increased thickness of endometrium (54 cases), myoma (102) and polyp (131). In the first cases, by hysteroscopy we observed a functional or hypertrophyc endometrium (37 cases, 68,52\%). 67,64\% of myomas and $62,59 \%$ of polyps were confirmed during hysteroscopy. In postmenopausal metrorrhagias, the ultrasound scan findings were increased endometrial thickness (194 cases), myoma (18) and polyp (98); in the cases of increased endometrial thickness, in the hysteroscopy an atrophic endometrium was observed in 110 cases (56,70\%). Only $38.80 \%$ of myomas were confirmed by hysteroscopy, whereas in the polyps' case $73 \%$ of cases were confirmed. Discussion: The positive predictive value of the ultrasound scan for the diagnosis of uterine myomas is significantly higher in the case of premenopausal metrorrhagias.

Key-words: diagnostic hysteroscopy, metrorrhagia, myomas.

\section{P10 24}

The third generation of Essure permanent birth control: have we improved?

J.B. Arjona Berral, E. Velasco Sánchez, J.J. Serrano Dávalos, J.E. Ríos Castillo, B. Povedano Cañizares

Hospital Universitario Reina Sofia, Córdoba, Spain

Introduction: Since the Essure device was approved by the FDA, three generations of microinserts have been devel- 
oped, with changes in the length and the way of placement, simplifying the procedure and establishing new indications. Objectives: To assess wether there are differences between the second and the third devices generation, in relation to time needed, placement success and in complications registered. Materials and Methods: Retrospective cohort study of 4000 consecutive patients divided in two groups: GroupI (3010 cases) with the second generation device, and groupII (990 cases) with the third generation device. All data were recollected from the database of our Hysteroscopic Outpatient Unit of the Hospital Universitario Reina Sofia.

Results: Since the introduction of the third generation Essure, the hysteroscopic time has been reduced from five minutes to three minutes of media values. The success rate has remained the same in the last years, being in $98,8 \%$ of cases. About complications, we have registered $0,9 \%$ of vasovagals syncopes in group I in front of $1,3 \%$ of cases in groupII. The expulsion rate was $0,5 \%$ for GroupI(18 cases) in front of $0,0 \%$ for GroupII. We have registered one case of migration for both groups, one pelvic disease for both groups, and no perforation other intramyometrial placement in GroupII ( 1 and 3 cases respectively for GroupI). Conclusions: The introduction of the third generation Essure device has improved the method, becoming it safer because of a reduction in the complications registered ligated to the expulsion of one of the devices, and it has decreased the time requested for the placement. Key-words: Outpatient Hysteroscopy, Essure.

\section{P10_25}

\section{Laser diode in outpatient hysteroscopy}

A. González Paredes, A. Rodríguez Oliver, J. Fernández Parra, I. Vico Zúñiga

Obstetrics and Gynaecology Clinical Service. "Virgen de las Nieves" University Hospital, Granada, Spain

Summary: Office hysteroscopic has progressed due to technical advances. We present the results of hysteroscopic surgery using laser diode.

Introduction: The need for surgical instruments for outpatient hysteroscopy has been covered so far by Versapoint technology. The use of laser has been generalized in various fields of surgery, such as vascular and urological surgery.

Methods: We examined 42 cases in which we have used the laser diode for outpatient hysteroscopy. We used three hysteroscopes: a 4,5 or $5 \mathrm{~mm}, 30^{\circ}$ hysteroscope with a $5 \mathrm{Fr}$ working channel (Karl Storz) and another $1.8-\mathrm{mm}, 0^{\circ}$, semi-rigid hysteroscope with a $7 \mathrm{Fr}$. operative channel (Versascope). All the procedures were performed using the transvaginal approach and without anesthesia. Surgical diode laser system used was Velas30BK. We operated at a wavelength of $980 \mathrm{~nm}$, using the fibre in contact with the tissue for incision and coagulation. The surgical technique is similar to that described for the bipolar electrode.

Results: The most common indication for hysteroscopy was abnormal uterine bleeding $(57,1 \%)$. Seven patients $(17.7 \%)$ consulted for infertility, while $26.2 \%$ remained asymptomatic. Surgical procedures were polypectomy (73.8\%), uterine septum resections (6 patients) and 2 myomectomy. The average size of the polyps was $1.4 \mathrm{~cm}(0.5$ to $2 \mathrm{~cm})$. In the rest we used laser for biopsy and endocervix enlargement. We observed reduced patient discomfort during the operative part of hysteroscopic procedure, mainly in septum resection because it avoids myometrial stimulation. We observed vasovagal reaction in $2,4 \%$.

Discussion: Laser technology can be useful because it is well tolerated, with a minimum rate of complications, and according to our experience, it is an easy to learn procedure. Key-words: laser diode, office hysteroscpy, hysteroscopic surgery.

\section{P10_26}

\section{Symptomatic vs. asymptomatic women treated with Tamoxifen: hysteroscopic findings}

C. Marques, M. Simões, A. Gonçalves, S. Barreto, A.P. Pereira

Maternidade Dr. Alfredo Costa, Portugal

Objective: To compare hysteroscopic-histologic results in symptomatic versus asymptomatic women with endometrial thickening taking tamoxifen.

Introduction: Tamoxifen is a selective estrogen receptor modulator (SERM) that is widely used in the treatment of patients with breast cancer.

Materials and Methods: We reviewed the clinical data of 79 patients with breast carcinoma taking tamoxifen who underwent a hysteroscopy between January 2009 and June 2010. Two groups were formed: symptomatic (abnormal haemorrhage) (group 1, n1 =24) and asymptomatic women (group 2, $\mathrm{n} 2=55)$.

Results: Endometrial polyp was the most frequent hysteroscopic finding in both groups (group A - 46\% and group B - 40\%) and included the following histologic results: Group A - glandular polyp (73\%), typical hyperplasia (9\%), and adenocarcinoma (9\%); Group B - glandular polyp (68.2\%) and cystic atrophy (9.1\%). Diffuse endometrial thickening represented 29\% of hysteroscopic findings in Group A and $35 \%$ in Group B. The histology of this finding was: Group A - glandular polyp (28,6\%), typical hyperplasia (28.6\%), no abnormality (28.6\%); Group B - glandular polyp 
(10.5\%), atypical hyperplasia (5.3\%), no abnormality $(31.6 \%)$. No statistically significant differences were found between hysteroscopic findings in both groups.

Discussion: Endometrial polyp was the most prevalent diagnosis (hysteroscopy and histology) in both groups although the prevalence was slightly higher in the symptomatic one. The only adenocarcinoma diagnosed occurred in the symptomatic group; the presence of hemorrhage although limited by the small number of patients was not associated with a worse histerocopic/histological diagnosis. Key-words: tamoxifen, abnormal haemorrhage, hysteroscopy.

\section{P10 27}

\section{Endometrial thickness in women treated with Tamoxifen: hysteroscopic findings}

C. Marques, M. Simões, F. Ribeiro, A. Gonçalves, A.P. Pereira

Maternidade Dr. Alfredo Costa, Lisboa, Portugal

Summary: To evaluate the concordance between the hysteroscopic findings and histologic diagnosis in women with endometrial thickening treated with tamoxifen.

Introduction: Tamoxifen is a SERM with both agonist and antagonist activity. Its association with increased risk of endometrial cancer is known.

Materials and Methods: This retrospective study included patients with breast carcinoma treated with tamoxifen who underwent a hysteroscopy between January 2009 and June 2010 due to ultrasound abnormalities $(\mathrm{N}=79)$. Demographic, hysteroscopic and histologic results were analyzed.

Results: Ultrasound diagnosis of endometrial polyp (47.4\%) and diffuse thickening (52.6\%) was the main indication for hysteroscopy in our study group. The most frequent hysteroscopic finding was endometrial polyp (42\%) with the following histologic results: glandular polyp (69.7\%); typical hyperplasia (3\%) and cystic atrophy (6\%). Adenocarcinoma was found in one woman with endometrial polyp (3\%). Diffuse endometrial thickening represented 33\% of hysteroscopic findings and its histologic results were: glandular polyp (15.4\%), typical hyperplasia (7\%), and atypical hyperplasia $(3.9 \%)$. Septum $(6 \%)$ and synechiae $(6 \%)$ represented other hysteroscopic findings. In 13\% no abnormalities were found. Discussion: Endometrial polyps represent the most common endometrial pathology associated with tamoxifen exposure. Hysteroscopic-histologic correspondence was $69.7 \%$. Hysteroscopy should be considered the gold standard exam to evaluate the endometrium in women treated with tamoxifen.

Key-words: tamoxifen, endometrial thickning, hysteroscopy.

\section{P10 28}

Hysteroscopic findings in postmenopausal women taking Tamoxifen for breast cancer

A. Melo, C. Costa, J. Amaral, P. Bayer, M. Martinho Gynaecological Endoscopy Unit, Department of Obstetrics and Gynaecology, Hospital de S. João, Porto, Portugal

Summary: The aim of our study was to evaluate endometrial abnormalities in postmenopausal patients treated with tamoxifen as adjuvant therapy for breast cancer.

Introduction: Tamoxifen is a non-steroidal triphenylethylene derivate with clear antioestrogenic effects on the breast and known carcinogenicity in the gynecological reproductive organs. Women treated with tamoxifen are advised to undergo annual gynecological examination and ultrasonographic endometrial assessment. Hysteroscopy should be performed facing an abnormal uterine bleeding or endometrial thickening $\geq 8 \mathrm{~mm}$.

Materials and Methods: Retrospective analysis of hysteroscopies performed during a period of 12 months in an office gynaecological setting. Postmenopausal patients who were taking $20 \mathrm{mg} /$ day of tamoxifen were studied.

Results: Twenty-six patients were selected. The majority of our patients were asymptomatic $(\mathrm{N}=24,92,3 \%)$; only 2 women had postmenopausal uterine bleeding. Hysteroscopy was performed mainly because of endometrial thickening $(\mathrm{N}=20,77 \%)$ and suspicion of endometrial polyps $(\mathrm{N}=6,23 \%)$. In 6 patients $(23 \%)$ the hysteroscopy reveal no endometrial pathology and the most frequent abnormal findings were endometrial polyps $(\mathrm{N}=9,35 \%)$ and synechia $(\mathrm{N}=6,23 \%)$. We also diagnosed one submucous fibroid, one uterine septum, one case of focal hypertrophy and in 2 cases $(7,7 \%)$ the endometrial was atrophic. Surgical procedures were performed in 18 patients (69\%): 9 polypectomies, 1 resection of submucous fibroid, 5 endometrial biopsies, 1 synechiolysis and one hysterectomy. All polyps were confirmed by histology. Other histological findings were simple hyperplasia $(\mathrm{N}=4,15,4 \%)$ and 1 case of atypical hyperplasia $(3,8 \%)$. Tissue collection was too scant to give a diagnosis in 2 cases.

Discussion: Long term tamoxifen therapy is associated with increased risk of uterine pathology. Despite of being asymptomatic, $77 \%$ of our patients had some hysteroscopic abnormality and $69 \%$ of them underwent some surgical procedure. This results enhances the importance of routine evaluation of those patients and show that hysteroscopy should be considered the reference test to assess a thickened endometrium in women under tamoxifen.

Key-words: tamoxifen, endometrial thickening, hysteroscopy. 
P10 29

\section{Hysteroscopy in the diagnosis of asymptomatic postmenopausal women with sonographic endometrial thickening \\ A. Melo, C. Costa, J. Amaral, P. Bayer, M. Martinho \\ Gynecological Endoscopy Unit, Department of Obstetrics and Gynaecology, Hospital de S. João, Porto, Portugal}

Summary: The purpose of our study is to assess hysteroscopic and pathological findings in asymptomatic postmenopausal women with endometrial thickening.

Introduction: Endometrial carcinoma is the most common genital malignancy in women. Patients usually present with abnormal uterine bleeding, but $10-20 \%$ of women are asymptomatic. Endometrial thickening, measured by transvaginal ultrasonography, indicates an increased risk of malignancy and other endometrial pathology like polyps and hyperplasia.

Materials and Methods: Retrospective analysis of hysteroscopies performed during a period of 12 months in an office gynaecological setting. Patients were selected according to menopausal state, absence of uterine bleeding and endometrial thickness above $4 \mathrm{~mm}$.

Results: One hundred and forty postmenopausal patients presented with endometrial thickening; $30 \%$ of them had abnormal uterine bleeding and were excluded; so, only 97 patients were studied. Mean age was 63 years $(47-85)$. The most frequent hysteroscopic finding were endometrial polyps $(\mathrm{N}=59,61 \%)$, focal hypertrophy $(\mathrm{N}=10,10,3 \%)$ and synechia $(\mathrm{N}=10,10,3 \%)$ and in 4 cases we had hysteroscopic suspicion of malignancy $(4,1 \%)$. In $53 \%$ of patients we performed polipectomy and in $19,6 \%$ endometrial sampling was collected; 3 patients $(3,1 \%)$ underwent hysterectomy and one (1\%) was submitted to resection of submucous fibroid. Histology showed a normal endometrium in $5,2 \%$ of the patients and non atypical hyperplasia in $39 \%$ of them. Fifty two of the 59 suspected hysteroscopic polyps were confirmed by pathology and we found only one case of endometrial carcinoma (1\%). Hysteroscopic sensibility and specificity for detecting adenocarcinoma was 100 e 97\%, respectively.

Discussion: Hysteroscopy is a cost effective technique and allows directed visualization of the uterine cavity and endometrial targeted sampling collection. An evaluation plan using transvaginal ultrasonography as the initial screening, followed by office hysteroscopy with endometrial sampling, is the standard care for patients at higher risk of endometrial pathology.

Key-words: tamoxifen, postmenopausal women, hysteroscopy.
P10 30

Office hysteroscopy: one year experience of a tertiary care center

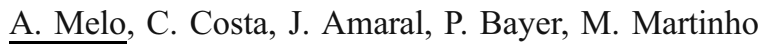

Gynaecological Endoscopy Unit, Department of Obstetrics and Gynaecology, Hospital de S. João, Porto, Portugal

Summary: The aims of our study were to assess indications for hysteroscopy and describe major hysteroscopic findings and procedures performed during a year of activity at the hysteroscopic unit of our hospital. Introduction: Office hysteroscopy is the gold standard procedure to study the uterine cavity. Using an atraumatic approach and mini-hysteroscopy it's possible to accurately diagnose and, at the same time, to treat intrauterine pathology safely in an office setting, with excellent patient tolerance.

Materials and Methods: Retrospective analysis of hysteroscopies performed during a period of 12 months in an office setting. Diagnostic procedures were performed with a 2,9 $\mathrm{mm}$ hysteroscope without systematic local anesthesia or cervical preparation. A $5 \mathrm{~mm}$ surgical hysteroscope, mechanical instruments and Versapoint ${ }^{\circledR}$ electrode were used for surgical procedures and saline for distention.

Results: A total of 848 hysteroscopies were performed. Those conducted for hysteroscopic sterilization or for second look of uterine cavity after a previous surgical procedure were exclude from our study. Thus, only 509 cases were considered. The mean age of the patients was 52 years (21-87 years) and $48 \%$ were postmenopausal. Thirty four percent had abnormal uterine bleeding. The most frequent indications were endometrial thickening (38\%), sonographic suspicion of endometrial polyps (35\%) or submucous fibroids $(7 \%)$, infertility disorders $(8 \%)$ and postmenopausal bleeding $(3,7 \%)$. In $22 \%$ of the cases the examination was normal and most frequent abnormal hysteroscopic findings were endometrial polyps $(39 \%)$, focal endometrial hypertrophy $(11 \%)$, submucous fibroids $(8,6 \%)$ and synechia $(6 \%)$. In $5 \%$ of the cases our findings were suggestive of endometrial carcinoma. In 138 women $(27,2 \%)$ we purely performed a diagnostic procedure. Surgical procedures included mainly polypectomy $(48,5 \%)$, endometrial biopsy $(34,8 \%)$, myomectomy $(7,8 \%)$ and synechiolysis $(2,7 \%)$. In the studied population, $5(0,9 \%)$ cases of atypical hyperplasia and $19(3,7 \%)$ cases of carcinoma were detected. Major histologic findings were endometrial 
polyps $(15 \%)$, non atypical hyperplasia $(27,9 \%)$ and uterine fibroids $(4,1 \%)$. There were no significant complications.

Discussion: In our experience, hysteroscopy is a safe procedure that allows diagnosis and treatment of a significant number of benign intrauterine pathology in an office setting with minimal discomfort to the patient.

Key-words: office hysteroscopy, intrauterine pathology, uterine cavity.

\section{P10 31}

\section{Office hysteroscopic uterine septum resection and pregnancy}

I. Vico Zúñiga, A. González Paredes, A. Oliver Rodríguez, J. Fernández Parra

Obstetrics and Gynaecology Clinical Service. "Virgen de las Nieves" University Hospital, Granada, Spain

Summary: Uterus septus is the most common congenital uterine anomalies caused by insufficient resorption of the müllerian ducts. We evaluated reproductive outcomes after hysteroscopic metroplasty of uterine septa.

Introduction: Septate uterus is a congenital disorder uterine that increases the incidence of recurrent miscarriages and obstetric complications. Hysteroscopic metroplasty is the gold standard for assessing uterine septa.

Methods: We recruited 39 patients, and we performed uterine septum resection using an office hysteroscopic procedure. We used 4,5 or $5 \mathrm{~mm}$ continuous flow, $30^{\circ}$ hysteroscope (Karl Storz). All the procedures were performed whithout cervical tenaculum and speculum, without anesthesia and during the proliferative phase, using versapoint system or laser diode.

Results: We trated a group of patients between 26-44 y-o. They consulted for sterility (16/39), recurrent miscarriage (13/ $39)$, hypermenorrhea (2/39) and 8 of them were asyptomatics. The average operating time was 395 seconds and no important complications occurred during the procedure. The diagnosis was uterine septum in $41 \%$ and the $59 \%$ had subseptate uterus. After metroplasty 4 patients had a miscarriage, and 18 achieved evolutive pregnancie (11\% IVF-ICSI), 5 of them are now in progress. Termination was by caesarean section in $53.9 \%$ and the $46,1 \%$ were preterm births, three of them caused by preterm premature rupture of membranes. The average delivery gestational age was 35 weeks.

Discussion: Office hysteroscopy is an important therapeutic weapon for the sterility-infertility related uterine malformation like uterine septum. It allows us to achieve evolutive pregnancies but we found a high rate of caesarean sections and prematurity.

Key-words: uterine septum, office hysteroscopy, metroplasty.

\section{P10 32}

\section{The recognition of vessels' bifurcations through hysteroscopy and its importance in diagnosis of endometrial cancer}

L. Lavasidis, F. Gkrozou, T. Vrekousis, G. Dimakopoulos, A.A. Vlachokosta, P.A. Asvestas, G.K. Matsopoulos, M.H. Paschopoulos

University of Ioannina, Greece

Summary: We study through hysteroscopy the vessels' bifurcations in certain pathological situations of endometrial cavity and also in normal endometrium. We identify differences between these cases, helpful in recognition of endometrial cancer comparing to the other situations.

Introduction: We aim to identify specific vessels' characteristics through hysteroscopy which are important in cases of endometrial cancer and have high sensitivity and specificity. Materials and Methods: One hundred five (105) patients underwent hysteroscopy. 77 cases concerned pathological situations such as endometrial cancer, adenomyosis, hyperplasia, DUB and polyps and 28 cases concerned normal endometrium. By using a new methology of studying vessels' characteristics, we calculated the bifurcations of vessels. Our study is retrospective.

Results: Analysis Of Variance (ANOVA) showed statistically significant differences in the mean number of bifurcations among different diagnoses. Multiple comparisons showed that the mean number in the case of cancer is significantly smaller than the ones measured in all other diagnoses but also normal endometrium. ROC curves were computed and AUC's were in all cases statistically significant ( $\mathrm{p}$ - level $=0.05$ ) and higher than 0.79 , reaching 0.99 .

Discussion: There are strong indications that the number of bifurcations can be a substantially informer marker with high sensitivity and specificity rate to discriminate endometrial cancer from other diagnosis, but also from normal endometrium. It is necessary to expand the number of women who underwent hysteroscopy and determine its diagnostic accuracy.

Key-words: hysteroscopy, vessels' characteristics, cancer.

\section{P10 33}

Review of malignant polips diagnosticated in our hysteroscopy service

C. Sisó Raber, R. Nonell, P. Jou, M. Cardona

Hospital Clinic Barcelona, Spain

Background: Endometrial polyps are very common lesions in women as age increases. Its prevalence is estimated 
between 10 to $24 \%$. In the literature the risk of malignancy keeps around $1 \%$.

Objective: Report the experience in our Hysteroscopy Service in detecting malignant endometrial polyps.

Materials and Methods: Of the 6496 office hysteroscopy done between January 2003 and May 2009 we describe the incidence and clinical features of patients diagnosed of endometrial polyp who were surgically treated by polypectomy and with histological confirmation of malignancy. Results: 1994 patients were diagnosed of endometrial polyp (30.7\%) and polypectomy were conducted in 1793 cases $(27.6 \%)$. Malignancy was found in 39 patients $(2,2 \%)$. The mean age of this group was 60.4 years (SD 16.0). Depending on the hormonal state we found 17 premenopausal $(35.4 \%)$ and 31 postmenopausal women $(64.5 \%)$. Reasons for medical consultation was bleeding in 36 cases $(75 \%)$, endometrial thickness increased in 10 cases $(21 \%)$ and study of a primary sterility in 2 cases $(4 \%)$. There were no statistically significant differences found depending on the hormonal condition and the clinical manifestation (Chisquare $=1.49 ; \mathrm{p}>0,10)$. Hysteroscopic polypectomy was done in a office setting in 16 women $(41 \%)$, in the operating room in 23 women $(59 \%)$. No complications were reported in any polipectomy of malignant polyps.

Conclusion: We found signs of malignancy in 2,2\% of the polyps resected. Based on this malignancy rate and to simplicity and safety of hysteroscopyc polypectomy, our suggestion is to remove all diagnosed polyps, independently of the clinical manifestation either the age of the patient. Key-words: hysteroscopy, endometrial polyps, malignancy.

\section{P10_34}

The examination of endometrial cavity through hysteroscopical identification of vessels' characteristics

A.A. Vlachokosta, P.A. Asvestas, F. Gkrozou, L. Lazaros, T. Vrekousis, M.H. Paschopoulos, G.K. Matsopoulos University of Ioannina, Greece

Summary: We study the endometrial cavity by examining hysteroscopically morphological vessels' characteristics. Our study is retrospective. In order to confirm vessels' characteristics and different pathological situations or normal endometrium, we knew the result of biopsy.

Introduction: We aim to examine morphologic alterations between pathological and normal endometrial images by means of advanced image processing techniques.

Materials and Methods: One hundred and five (105) patients who underwent hysteroscopic assessment of the endometrium were analyzed. Seventy seven (77) of them were diagnosed as being pathological and twenty eight (28) of them as being normal. The proposed methodology involved the extraction of vessel centerlines by means of differential calculus and the estimation of vessel's features. At each pixel that belonged to a centerline of vessel, the diameter and the orientation of the vessel were estimated. Furthermore, the length of each vessel was estimated and the total number of bifurcations, and vessels were counted. Finally, some texture features (fractal dimension, correlation dimension, lacunarity) were estimated.

Results: The mean value and standard deviation for each feature were computed and furthermore the corresponding histograms were created for both pathological and normal cases. The results indicated strong discrimination between pathological and normal cases with respect to the fractal dimension.

Discussion: Since the fractal dimension measures the roughness of the texture of an image, the observed differences indicate that pathological endometrial images present different texture patterns. Therefore, the fractal dimension could be used as an index for assisting the diagnosis of endometrial pathological cases.

Key-words: hysteroscopy, vessel's characteristics, fractal dimension.

\section{Poster Session 11_Oncology}

\section{P11_1}

The effect of BMI in patients with endometrial cancer managed by laparoscopy

J. Gilabert-Aguilar, J. Gilabert-Estellés, S. Costa, R. Aliaga, F. Coloma, J.J. Ródenas

Hospital Arnau de Vilanova, Spain

Obesity has been considered a technically challenging situation for the laparoscopic treatment of patients with endometrial cancer, as concerns have been raised about its outcomes in this setting. A retrospective cohort of 142 patients with stages I-II of endometrial cancer was classified according to body mass index (BMI) at the time of surgery in non-obese (group 1, BMI $<30 ; n=42$ ), obese (group 2, BMI 30-40; $n=76$ ) and morbidly obese patients (group 3, BMI $>40 ; n=24$ ). All these cases were treated by laparoscopic hysterectomy with bilateral salpingooophorectomy, and additional pelvic and paraaortic lymphadenectomy were performed according to the clinical stage and intraoperative evaluation. Intraoperative outcomes did not differ amongst groups, even though patients in group 3 had a higher incidence of previous co-morbidity. Rates of operative complications did not significantly differ between strata of BMI except for higher transfusion rate in morbidly obese patients. A pseudonodular pattern, assessed by diagnostic 
hysteroscopy, was more frequent in morbidly obese patients than in those assigned to the other groups. Moreover, no grade3 tumour and no positive peritoneal cytology was detected in group 3. Over a mean follow-up of 58.1 months, recurrence rates and the need of a postoperative treatment were lower in the morbidly obese group.

Key-words: endometrial cancer, morbid obesity, laparoscopic lymphadenectomy.

\section{P11_2}

\section{Comparison of laparoscopic and laparotomic surgery for borderline ovarian tumor}

J.-H. Kim, J.-Y. Park, D.-Y. Kim, Y.-M. Kim, Y.-T. Kim,

J.-H. Nam

Department of Obstetrics and Gynaecology, University of Ulsan College of Medicine, Asan Medical Center, Republic of Korea

Objectives: To compare the surgical and survival outcomes after surgery by laparoscopy or laparotomy for borderline ovarian tumors.

Materials and Methods: Retrospective analysis was performed on three hundred thirty six patients with borderline ovarian tumor who underwent laparoscopic or laparotomic surgical management.

Results: Of 336 patients with borderline ovarian tumor, 245 patients underwent laparoscopic surgery and 91 patients underwent laparoscopic surgery from 1997 to 2009. Age, parity, and body mass index was similar between the two surgery groups. Menopausal women were more in laparoscopy group. Mean tumor size was higher in laparotomy group. FIGO stage, histologic type, bilaterality of tumor, microinvasion, micropapillary pattern, invasive implant, intraoperative tumor rupture, malignant ascites or cytology, and preoperative CA 125 level were not different between the two surgery groups. However, conservative management, use of ovarian cystectomy were more common in laparoscopy group, and comprehensive surgical staging including lymph node dissection and omentectomy were more common in laparotomy group. After mean follow-up time of 56 months (range, 3-132 months), recurrence rate $(5.1 \%$ vs $4.2 \%, \mathrm{p}=0.763)$ and death rate $(1.7 \%$ vs $1.4 \%, \mathrm{p}=$ 0.864 ) were not different between the two surgery groups. Five year disease-free survival was $94 \%$ and $98 \%$, respectively $(\mathrm{p}=0.8827)$, and 5 -year overall survival rate was $98 \%$ in both groups ( $\mathrm{p}=0.9643$ ).

Conclusion: Laparoscopic surgery for borderline ovarian tumor was feasible in most cases. Although comprehensive staging procedure was less frequently used and conserva- tive management was adopted more frequently, the survival was not compromised. Laparoscopic surgery may be a safe and reasonable alternative to laparotomic surgery in the management of borderline ovarian tumor.

Key-words: borderline ovarian tumor, laparoscopic surgery, staging operation.

\section{P11 3}

High body mass and laparoscopic approach

N. Martínez, E. Martínez, C. Canseco, S. Heron, C. Medina, M. Guzmán, A. Zapico

Universitary Hospital Príncipe de Asturias. School of Medicine. Alcalá University, Madrid, Spain

Objective: To evaluate the different surgical approaches and morbidity in patients with Body Mass Index (BMI) over $40 \mathrm{~kg} / \mathrm{m}^{2}$.

Materials and Methods: A retrospective study between 1997 and 2009, over 16 consecutive patients with endometrium cancer and BMI over 40 were studied. Two groups were defined, whether laparoscopic (LPS) or laparotomic (LPM) approach were used. We have studied different items such as epidemiological data, diagnosis procedures, surgical access, operating time, conversion rate, complications rate, hospital stay, transfusion rate, pathological findings, FIGO stage and survival rate. Statistical analysis was done using SPSS 15.O.

Results: Mean age was LPS 62,40+2,80(49-75) vs LPM 66 $+4,28$ (52-81)years and mean BMI LPS 45,27+1,33 $(40,27-52,20)$ vs LPM $42,87+1,23(41,11-49) \mathrm{kg} / \mathrm{m} 2$. No differences were observed. Endometrial risk factors were seen in $12(75 \%)$ patients. The initial surgical access was LPS in 10 patients $(62,5 \%)$ and LPM in the remaining 6 cases $(37,5 \%)$. Lymphadenectomy was possible in $7(70 \%)$ of LPS group vs $2(33,3 \%)$ cases in LPM group. Conversion into laparotomy was necessary in $2(20 \%)$ patients mainly due to anesthesical problems. Hospital stay was shorter for LPS 6,78+1,35 (2-15) vs LPM 11,50+4,42 (4-33)days $(\mathrm{p}<0.001)$. Haemoglobin balance was: LPS $2,11+0,90(0,7-3,8)$ vs LPM 3,10+0,26 $(0,8-5,2) \mathrm{gr} / \mathrm{dl} . \mathrm{p}<$ 0.001 Global transfusion rate was $3(18,75 \%)$; no differences were achieved between both groups. Nodes collected were $13.06+1.44(2-21)$ LPS vs $12.52+2.2(2-21)$ LPM ( $p=$ $0.47)$. Survival rate was similar $(\mathrm{p}=0.29)$.

Conclusions: BMI should not be considered as a contraindication for the laparoscopic approach. In addition succesful laparoscopic staging shows lower hospital stay and morbidity. Key-words: Body Mass Index, laparoscopy, endometrium cancer. 


\section{P11 4}

Laparoscopic lymphadenectomy in early stages of endometrium cancer, a retrospective review

A. Zapico, E. Martínez, P. Fuentes, F. Arnanz, J. González, A. Couso, N. Martínez

Universitary Hospital Príncipe de Asturias. School of Medicine. Alcalá University, Madrid, Spain

Objective: To evaluate the endometrium cancer surgical results in early stages with a complete FIGO laparoscopic approach. Materials and Methods: A retrospective study between 1996 and 2009, over 76 consecutive patients with endometrium cancer and new surgical stage Figo 2009 IaG1G2 . All patients had a laparoscopic (LPS) approach. We have studied different items such as epidemiological data, diagnosis procedures, surgical access, operating time, conversion rate, complications rate, hospital stay, transfusion rate, pathological findings, FIGO stage and survival rate. Statistical analysis was done using SPSS 15.O.

Results: Mean age was $61.04+1.10(36-82)$ years and mean BMI $31.34+0.67(17,57-52,20) \mathrm{kg} / \mathrm{m}^{2}$. Endometrial risk factors were seen in $53(69.73 \%)$ patients. The initial surgical access was LPS in 76(100\%) patients. Lymphadenectomy was performed in all cases. Conversion into laparotomy was necessary in $8(10.5 \%)$. Operating time time was LPS $154.07+3.85(50-285)$ minutes. Hospital stay was LPS 5.81+1.1(2-65) days. Postoperative complications were 18(23.68). Haemoglobin balance was 2,65+0,13(0,4$8,8)$.Global transfusion rate was 5 cases $(6.6 \%)$. Nodes collected were $14,06+1,44(2-22)$. Survival rate at 5 th year was $70(92.11 \%)$. Median follow up was $39.70+3.90$ (82114) months. Medical causes of death were described in all cases without cancer relation.

Conclusions: Laparoscopic approach is feasible in endometrium cancer. Althought in succesful laparoscopic staging morbidity rate was small, lymphadenectomies could be avoid in most of the early stages because of the good prognosis of these patients

Key-words: lymphadenectomy, laparoscopy, staging endometrium cancer.

\section{P11_5}

Advantages of laparoscopic approach in endometrium cancer: our experience

E. Martínez, A. Zapico, N. Martínez, R. Cajal, P. Garbayo, D. Martínez, L. Gonzalez

Universitary Hospital Príncipe de Asturias. School of Medicine. Alcalá University, Madrid, Spain
Objective: To evaluate the surgical approaches, advantages and morbidity in patients with endometrium cancer.

Material and Methods: A retrospective study between 1996 and 2009, over 228 consecutive patients with endometrium cancer. Two groups were defined, whether the laparoscopic (LPS) or laparotomic (LPM) approach were used. We have studied different items such as epidemiological data, diagnosis procedures, surgical access, operating time, conversion rate, complications rate, hospital stay, pathological findings, FIGO stage and survival rate. Statistical analysis was done using SPSS 15.O.

Results: Mean age was $62.36+0.65$ (36-86) years and mean BMI $31.15+0.42(17.57-52.20) \mathrm{kg} / \mathrm{m}^{2}$. No differences were observed. Endometrial risk factors were seen in 125 (54.48\%) patients. The initial surgical access was LPS in 165 patients $(72.36 \%)$ and LPM in the remaining 63 cases $(27.63 \%)$. Lymphadenectomy was possible in $41(61.07 \%)$ of LPM vs $145(87.88 \%)$ cases in LPS group. Conversion into laparotomy was necessary in 17 (7.5\%). Operating time time was longer ( $\mathrm{p}$ $<0,01)$ for LPS $154.07+3.85(50-285)$ vs LPM $142.06+5.99$ (45-270) minutes. Hospital stay was shorter for LPS 5.61+0.63 (2-65) vs LPM 7.76+0,68(3-33) days ( $\mathrm{p}<0.05)$. Postoperative complications were $25(15.15 \%)$ LPS vs $18(28.57 \%)$ LPM group. Haemoglobin balance was: LPS $2,89+0,13(0,4-8,8)$ vs LPM 3,16+0,2(0,4-7,8 gr/dl.p $<0.001$. Nodes collected were LPS $14,06+1,44(2-22)$ vs LPM 13,52+2,2(2-21) $(\mathrm{p}=0.47)$. Survival rate was similar $(\mathrm{p}=0.29)$.

Conclusions: LPS approach is feasible in endometrium cancer. In addition succesful laparoscopic staging shows lower hospital stay and morbidity. In our hospital laparoscopy approach is considered the first option for access in endometrium cancer.

Key-words endometrium cancer, laparoscopy, advantages.

\section{P11_6}

Laparoscopic management of early stage epithelial ovarian and fallopian tubal cancer

J.-Y. Park, D.-Y. Kim, J.-H. Kim, Y.-M. Kim, Y.-T. Kim, J.-H. Nam

Department of Obstetrics and Gynaecology, University of Ulsan College of Medicine, Asan Medical Center, Republic of Korea

Background: To compare feasibility, accuracy and safety of laparoscopy and laparotomy in surgical staging of early stage ovarian and fallopian tubal cancer.

Methods: Outcomes of patients with stage I ovarian and fallopian tubal cancer who underwent complete surgical 
staging at Asan Medical Center, Korea between 2004 and 2008 were retrospectively evaluated.

Results: Forty patients were surgically staged through laparoscopy and 76 through laparotomy. There were no between-group differences in mean age, parity, body mass index, lymph nodes retrieved, and omentum specimen size, nor were there between-group differences in the percentage of patients who were postmenopausal, those referred for restaging, in the time interval to restaging, in those upstaged after surgery, and in those with intraoperative tumor rupture. The laparoscopy group had significantly shorter operating time $(230 \pm 93 \mathrm{~min}$ vs. $278 \pm 61 \mathrm{~min}, \mathrm{p}=$ $0.001)$, less blood loss $(301 \pm 217 \mathrm{~mL}$ vs. $494 \pm 380 \mathrm{~mL}$, $\mathrm{p}=0.004)$, less transfusion requirement $(15 \%$ vs. $30.3 \%, \mathrm{p}=$ $0.071)$, faster return of bowel movement ( $1.7 \pm 1.3$ days vs. $3.6 \pm 1.7$ days. $\mathrm{p}<0.001)$, and shorter postoperative hospital stay $(7.9 \pm 4.7$ days vs. $14.5 \pm 5.6$ days, $\mathrm{p}=0.002)$ and time interval to adjuvant chemotherapy (12.8 \pm 4.9 days vs. $13.9 \pm$ 7.8 days, $\mathrm{p}<0.001)$. There were no postoperative complications requiring further management. After a median followup time of 34 months (range, 4-63 months), 5 year disease free survival rate was $92 \%$ and $93 \%(\mathrm{P}=0.876)$ and 5 year overall survival rate was $96 \%$ and $94 \%(\mathrm{P}=0.841)$ for laparoscopy and laparotomy group, respectively.

Conclusions: Laparotomy and laparoscopy showed similar surgical staging adequacy and accuracy, and laparoscopy showed more favorable operative outcomes. Laparoscopy was safe for early stage ovarian and fallopian tubal cancer. Key-words: epithelial ovarian cancer, laparoscopic surgery, staging operation.

\section{P11_7}

One port surgery of ovarian tumors for 5 years personal experience : the laparoscopy-assisted mini-laparotomic ovarian resection (GLAMOR)

J.H. Yoon, S.H. Yoo*

Department of Obstetrics and Gynaecology, Catholic University Medical College, South Korea; *Department of Anesthesiology, College of Medicine, Soon Chun Hyang University, Chunan, South Korea

Objective: Recently, development of optical and laparoscopic instruments is able to substitute laparoscopic surgery for open abdominal surgery in gynaecologic surgery. But, laparoscopic surgery has some limitations, for example skillfullness of operator, size of the lesion. In surgery of malignant ovarian tumor, there are various complications such as rupture of tumor, spillage, incomplete resection of lesion, metastasis induced by chimney effect etc. That reason makes the mini-laparotomy gain power, which overcomes the disadvantages of laparoscopic surgery. So, we tried the one port only surgery and reported that experience.

Methods: We review the charts of 22 cases who had huge ovarian mass between April 2005 and October 2009.

Results: 22 patients with huge ovarian mass, which initially was not laparoscopically resectable. Following imaging and baseline work-up ( ultrasonogram,abdominal CT,MRI,PET$\mathrm{CT}$ etc.), the patient underwent the gasseous laparoscopy assisted mini-laparotomic ovarian resection (GLAMOR) with or without hysterectomy. The mean diameter of tumor was about $42.2 \pm 9.6 \mathrm{~cm}$. The median time of surgical procedure lasted about $277.8 \pm 62.5$ minutes in average. The mean volume of blood loss was about $125.6 \pm 177.5 \mathrm{ml}$. No major intraoperative or postoperative complications occured. Some patient also underwent chemo-therapy. Follow-up was performed so far.

Conclusions: This experience suggests that such a treatment process is possible. The mini-laparotomy, minimal incision, can give relieve of pain and early recovery through minimization of tissue and nerve damages. Nevertheless, the narrow visual field of mini-laparotomy can't provide sufficient vision of internal abdominal space, that makes operator get limited vision. So, we report case series that huge ovarian tumor was successfully removed by the combination of gasseous laparoscopy and mini-laparotomy (GLAMOR), the limitation of which were conquered. GLAMOR potentially allows or decrease perioperative morbidity and blood loss, faster recovery and better cosmetic results. Large studies with long term follow-up are needed to confirm the effectiveness of this approach.

Key-words: gasseous laparoscopy assisted mini-laparotomic ovarian resection (GLAMOR), one port surgery, huge ovarian mass

\section{P11_8}

A clinical study of laparoscopic radical hysterectomy with paraaortic and pelvic lymphadenectomy

P. Jang, Y. Kwon

Ob/Gyn, Hallym University School of Medicine, Republic of Korea

Objective: To explore results of laparoscopic radical hysterectomy and lymphadenectomy in patients with cervical carcinoma.

Methods: At our hospital, a total of 40 laparoscopic radical hysterectomy and lymphadenectomy procedures were performed in patients with cervical carcinoma. We reviewed the clinical data of patients who underwent laparoscopic radical hysterectomy and lymphadenectomy. 
Results: All surgical procedures were completed laparoscopically. Paraaortic lymphadenectomy was performed in 8 patients and pelvic lymphadenectomy was performed in all patients. Minor complications occurred in 4 of 40 patients. The overall conversion rate was $4 / 40$, consisting of 3 emergency and 1 elective conversions. Operative cystotomies occurred in 3 patients and all were repaired laparoscopically. One patient underwent laparotomy because of ureteral injury. One patient had a ureterovaginal fistula after the operation that required reoperation.

Conclusions: Laparoscopic radical hysterectomy in combination with lymphadenectomy is becoming a routine procedure in gynaecologic oncology.

Key-words: laparoscopy, cervical cancer, fistula.

\section{P119}

\section{Laparoscopy in the management of pathological adnexal cysts \\ W. MacNab, T. Majumudar, H. Abdel-Rahman \\ Hinchingbrooke Healthcare NHS Trust, UK}

Summary: The use of RMI is an effective tool for triaging the management of adnexal cysts in women with RMIs of $<25$ and $>250$. In women with RMIs of 25-250 the use CT routinely or selectively in those with US scores of 3 may help in improving our ability to select cases which can be done laparoscopically.

Introduction: To evaluate the use of Risk of Malignancy Index (RMI) and appropriateness of laparoscopic surgery in the management of adnexal cysts.

Materials and Methods: Prospective study of 61 women presenting to a peripheral cancer unit. All women had ultrasound imaging + CA125 levels and their management triaged according to their RMI.

Results: In women with RMI $<25$ no ovarian cancers were diagnosed. $54.2 \%$ had serial follow-up scans and were discharged after 1 year as the cyst did not change in size and appearance. $33.3 \%$ had laparoscopic surgery. In women with RMI of 25-250 the incidence of malignancy was $14.3 \%$. $64.3 \%$ women in this group had surgery. Half were done laparoscopically and 2 ovarian cancers diagnosed. These cases were inadequately staged and had iatrogenic cyst rupture at surgery. All women with RMI $>250$ had ovarian cancer. Those women managed surgically had a staging laparotomy at the cancer centre.

Discussion: This study confirms that in women with RMI of $>250$ the incidence of ovarian malignancy is very high and they should have surgery in regional cancer centres. The risk is low if RMI is $<250$ and most women can be effectively managed with laparoscopic surgery in a peripheral unit. Some women with an RMI of 25-250 will inevitably have ovarian cancer. Laparoscopic surgery in management of these women remains controversial due to incomplete staging and risk of iatrogenic cyst rupture at surgery.

Key-words: RMI, adnexal cyst, laparoscopy.

\section{P11 10}

\section{Over ten years follow-up of laparoscopic treatment} of borderline ovarian tumors

A. Dogan, H. Diebolder, O. Camara, I.B. Runnebaum Department of Gynaecology, Friedrich Schiller University, Jena, Germany

Summary: Based on our 10 years experience in laparoscopic treatment of borderline ovarian tumours (BOT) we report favourable operative outcomes, $100 \%$ survival and minor complications. Fertility sparing surgery of women in reproductive age revealed as an appropriate treatment option in management of BOT.

Introduction: To date, all trials examining laparoscopic surgery of BOT have been relatively small, therefore we focused our study to validate the feasibility and safety of laparoscopic surgery.

Material and Methods: The medical reports of 45 patients with BOT treated with laparoscopic surgery in our hospital from 1996 to 2009, were retrospectively reviewed. Laparoscopic staging was performed according to the FIGO guidelines. 19 patients underwent a fertility-sparing surgery. Parameters such as tumour diameter, operating time, length of hospital stay, complications, and recurrence were evaluated. Results: The patients' mean age was 47 (18-78), diameter of the tumors was $60 \mathrm{~mm}(40-140 \mathrm{~mm})$ and CA 125 value was 31.8 (3.9-332). Mean follow up was 71 months. 9 conversions to laparotomy have been reported. 3 cases due to assessment of malignancy, 1 due to tumour volume, 3 due to FIGO III staging, 1 due to adhesions and 1 case because of a complication. Although the survival was $100 \%$, a tumour recurrence in 3 patients $(6 \%)$ has been diagnosed. No difference has been observed between laparoscopy and laparotomy regarding the access method. No major complications occurred when patients were treated laparoscopically. Discussion: The use of laparoscopic surgery still remains controversial due to accuracy of staging, intraabdominal tumor rupture and port site metastasis. Our study confirms laparoscopic surgery as safe and effective method in management of BOT.

Key-words: borderline ovarian tumor, laparoscopy. 
P11_11

Extraperitoneal laparoscopic pelvic lymphadenectomy: initial results of a new technique

Ó. Sanz Martìnez, I. Alonso, M. Munmany, A. Torné, M. del Pino, P. Fusté, S. Martínez-Román, J.A. Lejárcegi, J. Ordi, J. Pahisa

Department of Gynaecology and Obstetrics. Hospital Clinic of Barcelona, Spain

Background and Objective: Aortical lymphadenectomy represents an important issue in the management of locally advanced cervical cancer because it facilitates prognostic information and allows the therapeutical planning of radiotherapy. In theory, the possible extension of disease to pelvic lymph nodes is covered with the radiotherapy application in the pelvis. Nevertheless, increasing evidence shows the benefit of the removal of enlarged pelvic lymph nodes observed in imaging techniques. That represents a debulky and allows a better disease control. Usually, after an excellent left extraperitoneal approach for aortical lymphadenectomy, surgeons ongo pelvic lymphadenectomy by a transperitoneal approach. So, this procedure improves the risk of adherences and complications with radiotherapy. In this initial series, we present the first cases of pelvic lymphadenectomy after aortical lymph nodes removal by an extraperitoneal approach in patients with locally advanced cervical cancer and enlarged pelvic nodes in the magnetic resonance image previous to surgery.

Patients and Methods: From June-2008 to June-2010 a pelvic dissection was attempted at the time of a left extraperitoneal approach for staging of locally or regionally advanced cervical cancer in 16 patients with enlarged pelvic nodes in preoperative magnetic resonance imaging (MRI) in the Clinic Hospital of Barcelona. The purpose was staging and debulking, but no to perform a systematic pelvic dissection in this group of patients candidates for external radiation therapy. Inclusion criteria were: locally or regionally advanced cervical carcinomas, enlarged or suspicious pelvic nodes at imaging tests, no enlarged aortic nodes at MRI or computerized tomography (CT-scan), no contraindication for laparoscopic surgery, and informed consent of the patient.

Results: Sixteen patients were included. Median age was 48.56 (range, 35-76 years). All of them had squamous cervical cancer. FIGO stage: 5 IIb bulky and $11 \mathrm{IIIb}$. Median maximum tumor diameter was $59.8 \mathrm{~mm}$ (range 50-70 mm). Mean body mass index was 27.12 (range 19.4-34-1). Removal of left pelvic enlarged lymph nodes was successfully performed in 13 of 16 cases. Mean number of obtained nodes was 4,31 (range 1-8) and 12,5\% were positive. Removal of right pelvic enlarged nodes was successfully completed in 10 of 13 cases. Median number of lymph nodes removed was 4,3 (range 1-7) and of those $11,6 \%$ were positive. Neither intraoperative nor postoperative complications occurred. In five patients the extraperitoneal pelvic access was not possible due to technical difficulties. Average postoperative hospital stay was 1.6 (range 1-3 days). No node recurrence in the pelvic area has been observed after an average follow-up of 14.7 (range 1-25) months.

Conclusions: The lateral extraperitoneal approach used for the staging of advanced cervical cancer can be extended to the pelvic area when enlarged nodes are present reducing the risk of adhesion formation if a transperitoneal route is performed in patients candidates for radiation therapy.

Key-words: extraperitoneal laparoscopic pelvic lymphadenectomy, cervical cancer.

\section{Poster Session 12_Operative Hysteroscopy}

\section{P12_1}

\section{Learning curve of hysteroscopic sterilization L. Barros, F. Sousa Hospital de Dona Estefânia, Lisboa, Portugal}

Introduction: Hysteroscopic sterilization with Essure ${ }^{\circledR}$ emerged in 2002 as an improvement to permanent contraception that avoids abdominal incisions and a general anesthesia.

Objectives: The purpose of this work was to evaluate the learning curve and the outcome of the first 61 cases performed by a surgeon with experience in operative hysteroscopy during a 3 year period. All the procedures were performed in an outpatient setting.

Results: Mean age of the patients was 38 (27-47). Regarding the preparation most patients $(80 \%)$ took only a non-steroid anti-inflammatory (NSAI) preoperatively, followed by 8 patients who were prescribed with a NSAI plus misoprostol for cervical dilatation. In $85 \%$ of the cases the insertion of the devices was trough vaginoscopy without using anaesthesia or sedation and the remaining cases with local anaesthesia. There were four failures of placement during the first 2 years (rate of successful placement $=93 \%$ ). Surgical time reduced as experience increased (in $20075,6 \%$ of the procedures had a duration under 15 minutes compared to $52 \%$ of the cases in 2009). Mean pain (in a scale of 0 to 10) associated with the 
hysteroscopic sterilization was $3.8(9,8 \%$ of the patients referred pain over 6). Sonographic or radiographic studies at the 3 month evaluation confirmed the correct bilateral placement of the devices in all patients. There was no report of pregnancies in these patients.

Conclusions: Essure ${ }^{\circledR}$ is a safe, minimally invasive method, highly achievable to a skilled surgeon in operative hysteroscopy.

Key-words: hysteroscopic sterilization, learning curve, Essure

\section{P12_2}

\section{A retrospective case-control study comparing hysteroscopic resection versus hormonal modulation in treating menstrual disorders due to isthmocele P. Florio, G. Gubbini, E. Marra, D. Nascetti, E. Azzolini, L. Sabbioni, F. Petraglia \\ Department of Pediatrics, Obstetrics and Reproductive Medicine, Section of Obstetrics \& Gynaecology, University of Siena, Italy}

Introduction: In a retrospective case-control study we compared the effectiveness of hysteroscopic correction and hormonal treatment to improve symptoms [postmestrual abnormal uterine bleeding (PAUB), pelvic pain localized in supra-pubic site] associated with isthmocele. Materials and Methods: Women $(\mathrm{n}=39$; mean age $\pm \mathrm{SD}, 35$ \pm 4.1 years) were subdivided in Group A [patients $(n=19)$ subjected to hysteroscopic surgery (isthmoplasty)] and, Group B [women $(n=20)$ undergoing hormonal treatment consisting of one oral tablet containing $0.075 \mathrm{mg}$ of Gestodene and $0.030 \mathrm{mg}$ of Ethynylestradiol for 21 days, followed by 7 days of suspension]. Resolution and/or improvement of menstrual disorders; patients degree of satisfaction with the treatment were measured 3 months later, by office hysteroscopy (Grop A) or phone call.

Results: PAUB and pelvic pain resolution was achieved in all patients: Group A had significant lower numbers of days of menstrual bleeding $(\mathrm{P}<0.001)$, prevalence of pelvic pain in the suprapubic area $(\mathrm{P}=0.04)$ and, higher degree of satisfaction $(\mathrm{P}<0.001)$ compared to Group $\mathrm{B}$.

Conclusions: resectoscopic surgery is a valid way to treat patients with symptoms of prolonged post-menstrual uterine bleeding caused by isthmocele. Data from the present study also indicate that resectoscopy may be the first choice because it is minimally invasive and yields good therapeutic results.

Key-words: caesarean scar defect, post-menstrual abnormal uterine bleeding, resectoscopy.

\section{P12 3}

The use of nomegestrol acetate in rapid preparation of endometrium before operative hysteroscopy in pre-menopausal women

P. Florio*, S. Luisi*, E. Azzolini*, P. Litta, S. Angioni**, L. Mereu $^{\circ}$, L. Mencaglia ${ }^{\circ}$, L. Sabbioni*, F. Petraglia*

*Department of Pediatrics, Obstetrics and Reproductive Medicine, Section of Obstetrics \& Gynaecology, University of Siena, Italy; **Division of Gynaecology, Obstetrics and Physiopathology of Human Reproduction, University of Cagliari, Italy; ${ }^{\circ}$ Centro Oncologico Fiorentino, Section of Gynaecology, Florence, Italy

Introduction: The presence of a thin endometrium has an important role in allowing the best conditions for hysteroscopic surgery. Here, we explored the efficacy of a 14day administration of nomegestrol acetate, a progestogen with high progestogen potency effects, in rapid endometrial preparation to operative hysteroscopy.

Materials and Methods: A total of 86 fertile women selected for operative hysteroscopy received for 14 days either $5 \mathrm{mg}$ day -1 of nomegestrol acetate $(n=43$; group $A)$ or $4 \mathrm{mgday}-1$ of folic acid ( $\mathrm{n}=43$; group $\mathrm{B})$, starting on day 1 of the subsequent menstrual cycle. Before treatments on days 12-14 of the menstrual cycle, all patients underwent endometrial thickness measurement; ultrasonography of the ovaries to measure the appearance of a dominant follicle; diagnostic hysteroscopy with endometrial biopsy; plasma estradiol (E2), progesterone (P), luteinising hormone $(\mathrm{LH})$ and follicle-stimulating hormone (FSH) levels measurements. On the day of surgery, patients repeated endometrial and ovarian ultrasonography and, E2, P, LH and FSH measurement.

Results: At enrolment, endometrial thickness, mean follicular diameter and E2, P, LH and FSH concentrations did not differ between groups. At the time of operative hysteroscopy (i.e., after 14 days' treatment) group $\mathrm{A}$, but not group $\mathrm{B}$, showed significant (all $\mathrm{P}<$ $0.001)$ reduction of endometrial thickness, mean diameter of dominant follicle, E2, P and $\mathrm{LH}$ concentrations. Endometrial preparation was judged more effective in group A than B, since the endometrial mucosa in all of the women of group A appeared to be very thin, hypotrophic, regular and pale.

Conclusions: Administration of nomegestrol acetate was effective in reducing endometrial thickness, also acting on the hypothalamus-pituitary-ovarian axis, thus allowing highly favourable operative hysteroscopic conditions.

Key-words: pregestins, endometrium, thickness. 


\section{P12 4}

Is the hysteroscopy the right choice for therapy of large placental remnants?

L. Hrazdirova, Z. Zizka, D. Kuzel

Ob/Gyn Dept., General University Hospital, 1st Faculty of Medicine, Charles University in Prague, Czech Republic

Introduction: We performed a prospective study evaluating hysteroscopical management of placental remnants diagnosed by ultrasound in patient with prolonged spotting. Materials and Methods: We scheduled for hysteroscopy 28 women with prolongated bleeding more than 6 weeks after delivery and where the ultrasound examination described the hyperechogenic content in uterine cavity. In all cases the $3 \mathrm{~mm}$ hysteroscopy confirmed the diagnosis of placental remnants. Only 2 patients accomplished the hysteroscopical resection with $3 \mathrm{~mm}$ Versapoint system. In 26 cases it was necessary due the size of remnants, to use the bipolar resectoscopic system. In 3 patients the procedure was interrupted for worsening of visibility of operating field and accomplished after another 2 weeks. All patients underwent the second-look office hysteroscopy after one month.

Results: The mean operating time was 15 minutes, the mean medium loss was $554 \mathrm{ml}$, never exceeded $1000 \mathrm{ml}$. All placental remnants were confirmed by histology. The second-look hysteroscopy has confirmed in 16 patient normal hysteroscopic finding. In 12 patients was still small residual tissue visible. Those 12 patients were repeatedly resected only with $3 \mathrm{~mm}$ Versapoint system. No intrauterine adhesions were described.

Discussion: The hysteroscopical resection with resectoscopic system is efficient method of choice for treatment of placental remnants diagnosed by ultrasound. It allows targeted treatment without risk of intrauterine adhesions, which is largely described after blinded instrumental revision. This study was supported by the Grant Agency of the Ministry of Health of the Czech Republic, Grant NR 10576-3

Key-words: hysteroscopy, placental remnants, puerperium.

\section{P12_5}

Risk factors and the recidivals of endometrial polyps after hysteroscopic polyp resection

C.A. Ionescu, D. Gheorghiu, I. Pacu, B. Davitoiu, M. Dimitriu, E. Bratila

UMF Carol Davila, Clinical Emergency Hospital Sf

Pantelimon, Bucharest, Romania

Introduction: Endometrial polyps are very frequent pathologic entity that can recidivate after a first treatment. The objective of our retrospective study was to evaluate the risk factors that are involved after a hysteroscopic polyp resection. Materials and Methods: A retrospective study of 145 patients in whom polypectomy by hysteroscopy was made during 2004-2007 with a follow up untill December 2009 in order to detect patients who present recurrence and estimate the principal risk factor involved. Of these patients 90 patients were premenopausal and 55 were postmenopausal. The main risk factors studied were: age, menopause symptoms. Tamoxifen therapy, polyp size, body mass index. Results: In the cases analyzed we found $12,5 \%$ recurrences. A greater percent of recidivals (30\%) were among patients taking Tamoxifen, in comparaison with patients who did not take $(9 \%)$. The age, menopause syptoms, the size of the polyps or body mass index did not influence semnificativly the reccurence.

Conclusions: Only the patients taking Tamoxifen have a major risk of recurrence after hysteroscopic polypectomy. Key-words: endometrial polyps, hysteroscopy, recurrence

\section{P12_6}

\section{Hysteroscopic sterilization: Department's Experience}

R.M. Pinto, N. Nogueira Martins, C. Costa, J. Damasceno Costa, A. Pipa, F. Nogueira Martins

Hospital Sao Teotonio EPE, Viseu, Portugal

Introduction: The placement of Essure ${ }^{\circledR}$ tubal microimplants as non-reversible contraception has become the initial method of choice in such situations at the authors' Department, namely due to cost-effectiveness issues and wider range of applicability. It has been repeatedly stated to have a continuously growing number of supporters, backed up by a high incidence of patient requests. It is a highly effective and extremely well tolerated procedure, with a fast recovery back to active life, expensing incisions or general anesthesia, and with particular potential in cases of morbid obesity and other high risk patients for general anesthesia. Material and Methods: The authors made a retrospective study, where they included all the patients who underwent Essure $^{\circledR}$ sterilization at their Department, in a period between December, 2006 and June, 2010. The follow-up appointment and control of these 400 women was done three months later by pelvic $\mathrm{x}$-ray and vaginal ultrasound examination. Whenever a doubtful or unsatisfactory x-ray was found, a hysterosalpingogram was conducted. In case of expulsion or difficulties in application, the patients were offered a second attempt to place the micro-implants or an alternative contraceptive method.

Results: Placement of the micro-implants was achieved in 391 $(97.75 \%)$ of the 400 patients in the study. 348 women have completed the 3-month control period and in 336 of those 
(96.6\%), the surgical procedure has been considered to be successful ( 9 patients chose another method after proven unsuccessful placement, 2 patients missed the follow-up appointment, and in 1 case there was bilateral tubal obstruction shown in the hysterosalpingogram). A second attempt for placement was tried in 14 patients and 11 of those attempts were successful (the remaining three integrate the above seven patients group, who opted for an alternative method). There were 5 expulsions (1.44\%): 2 were successfully placed on a second attempt and 3 patients chose a different method. 64 hysterosalpingograms were performed due to altered x-ray on control and 3 of those were found to be abnormal: 2 patients underwent a successful second placement and 1 patient decided to try a different method. There was one pregnancy case which occurred thirteen months after placement of the micro-implants. Both x-ray and vaginal ultrasound examinations were within the defined standards; the patient had a vaginal delivery at 39 weeks gestation of a live newborn weighting $2790 \mathrm{~g}$ and later decided to undergo laparoscopic sterilization.

Conclusions: In the controlled 348 women group, the authors had a global success rate of $96.6 \%$. The patient complaints are lower in progressively more experienced hands. There were no major complications in this group and there was one pregnancy so far.

Key-words: hysteroscopy, sterilization, Essure.

\section{P12_7}

\section{Operative office hysteroscopy}

A. González Paredes, J. Fernández Parra, A. Rodríguez Oliver

Obstetrics and Gynaecology Clinical Service. "Virgen de las Nieves" University Hospital, Granada, Spain

Summary: Office hysteroscopy is a minimally invasive intervention that can be used to diagnose and treat many intrauterine and endocervical problems. It allows direct visualization of the uterine cavity, to perform directed biopsy, removal of endometrial polyps, and treatment of submucous fibroids, septa and adhesions, being a welltolerated procedure, with minimal complications.

Introduction: Small diameter hysteroscopes and bipolar electrosurgical system have expanded operative office hysteroscopic indications. The purpose of the study was to analyze the indications of use, difficulties in hysteroscopic performance, surgical procedures frequency and complications of this procedure in the office setting.

Materials and Methods: We performed a descriptive study including 5000 hysteroscopic office-based procedures conducted between June 2003 and April 2009, excluding those patients who had Essure sterilization. We used three office hysteroscopes: a 4,5 or $5 \mathrm{~mm}$ continuous flow, $30^{\circ}$ hysteroscope with an incorporated 5 Fr. working channel (Karl Storz) and other 1.8-mm, $0^{\circ}$, semi-rigid hysteroscope with a disposable sheath with a 7 Fr. operative channel (Versascope). All the procedures were performed using the transvaginal approach, whithout cervical tenaculum and speculum, using saline distension medium and without anaesthesia. Intra-uterine surgical procedures were performed with scissors, graspers forceps and the Versapoint Bipolar Electrosurgical System with the Twizzle electrode. Results: The mean age of the women recruited was 50,6 years, $855(17,1 \%)$ were nulliparous and 2247 $(44,9 \%)$ were menopausal patients. We were unable to perform hysteroscopy in 117 women (2,34\%) because severe pain, cervical stenosis, or non-visualization of cervical canal. The most common indication for hysteroscopy was abnormal uterine bleeding (70,5\%). In 2937 cases $(60,1 \%)$ we performed a surgical procedure, being polypectomy the most frequent and the Versapoint bipolar electrode the instrument more used (84,1\%). All tissues were sent for histological diagnosis. In a little proportion of women $(1,4 \%)$ we observed clinical vasovagal reaction and only a $4 \%$ described severe pain during the hysteroscopy. The rate of pelvic inflamatory disease was $0,08 \%$. No uterine perforation occurred during the procedures.

Discussion: Hysteroscopy is a diagnostic and therapeutical tool which can be successfully performed in the outpatient setting being a well-tolerated procedure, with minimal complications. New and improved technology enables us see and treat in the same hysteroscopic procedure.

Key-words: office hysteroscopy, versapoint bipolar electrode, intrauterine pathologies.

\section{P12_8}

\section{Obstetric outcome after hysteroscopic resection of congenital uterine septum}

C.A. Ionescu, D. Gheorghiu, I. Pacu, M. Dimitriu, R. Rotaru UMF Carol Davila,Clinical University Hospital Sf Pantelimon, Romania

Introduction: Congenital uterine malformations have been associated with a poor obstetric outcome. The objectives of our study was to determine the reproductive otcome following hysteroscopic resection in our population.

Materials and Methods: We analyzed retrospectively the reproductive efficiency of the patients submitted to hysteroscopic resection of the uterine septum in our unit between January 2003 and December 2007.

Results: We have 29 women in this study. Their mean age was $28,6,8+/-4,6$ years. The mean duration of infertility was 
$30,2+/-12,2$ months. Patients have reccurent abortion $(n=$ $5)$, primary infertility $(\mathrm{n}=18)$, one first trimester abortion $(n=2)$, asyptomatic septum $(n=4)$. From the women enrolled in the study 22 pregnancies had occured in the past: 15 first trimester abortion, 3 second trimester abortion, 2 preterm delivery, 2 ectopic pregnancy. From the 24 patients interested to conceive 20 patients got pregnant. From the patients who had a pregnancy, 14 ended in term deliveries, 3 ended in preterm deliveries and 3 were lost from the study. The delivery route was based on obstetric indications.

Conclusion: Our results confirm that the hysteroscopic resection of the uterine septum has a beneficial effect on pregnancy outcome.

Key-words: uterine septum, hysteroscopic resection, obstetric outcome.

\section{P129}

The use of the SNOMED model in a real-time management application of an hysteroscopy surgery

A. Kollias, M.H. Paschopoulos, M. Poulos

Ioanian University, Department of Archives and Library, Greece

Summary: In this work, an hysteroscopy surgery management application is presented, that was designed based on the medical information standard SNOMED, interconnected with a special way of satisfying the needs of the above examination, and moreover the way the medical information that existed in handwritten form are transmitted in the application's database in an effective way, is described.

Materials and Methods: The main aim was to approach and link both the medical incident and its digital format, in a way that follows the requirements of the medical standard while not affecting the process of solving the medical incident. The incorporation in the application's database, not the entirely SNOMED standard, but selected parts lists, groups or individual data of records strictly related to the hysteroscopy was chosen. Also the recording of consolidated coded primary information in the database instead of mapping information between the database and SNOMED was preferred. Thus achieved both greater functionality and more speed of the recording procedure, after avoiding lengthy interim computing matching conditions. Moreover the possibility of exchange information in CDA messages format for interoperability with other applications has been added.

Results: The Application computerizes the medical facts of an hysteroscopy surgery in real time without affecting its normal operation and simultaneously enables data analysis from a prospective researcher.

Discussion: The different way of using the SNOMED standard, free of generalities but fully respecting its rules, creates possibilities for broader and faster implementation of the standard. The creation of a remote network of accumulated information regarding the specific medical procedure is feasible and allows the meaning and sharing of medical information of hysteroscopy in a broader level.

Key-words: operative hysteroscopy, network, database.

\section{P12 10}

Comparative study between D\&C and hysteroscopy resection in the treatment of endometrial polyps

W. Pinheiro, A.K.C. Pereira, P.V. Gherpeli, J.M. Soares Jr., E.C. Baracat

University of São Paulo, Brazil

The aim of the current study is to comparatively evaluate the therapeutic effectiveness of endometrial polyps treatment applying two distinct techniques: curettage vs hysteroscopic resection.

Methods: Comparative study between two groups of 50 patients, distributed at random who showed endometrial polyps in the diagnostic hysteroscopy. The group i, 50 patients were submitted to polipectomy by hysteroscopic resection. The group ii, 50 patients submitted to D\&C. After 60 days a new hysteroscopic control was realized to evaluate the persistency or not of the polyps. The following parameters were analyzed; 1. principal indication of diagnostic hysteroscopy, 2. number of polyps, 3. association with myomas or endocervical polyps, 4. complications, 5. hystologic diagnosis, 6. hysteroscopy control.

Results: The study showed that in group ii there was $65 \%$ of persistency of endometrial polyps on the hysteroscopy control. Conclusions: The uterine D\&C was overcome in both diagnostic and therapeutic aspects through hysteroscopy technique considering the last one as the gold standard in the diagnosis and treatment of endometrial polyps.

Key-words: curettage, hysteroscopy, endometrial polyps.

\section{P12 11}

Correlation between parity and tolerance of Essure ${ }^{\circledR}$ procedure without anaesthesia

A. Agostini, M. Petrovic, S. Rua, S. Poizac, C. Couteau, M. Gamerre

La Conception Hospital, Marseille, France 
Summary: Influence of parity and number of vaginal delivery on Essure procedure tolerance was evaluated. No correlation between number of vaginal delivery and tolerance was found.

Method: From 1 June 2007 through 1 June 2010, 140 patients underwent tubal hysteroscopic sterilization with Essure ${ }^{\circledR}$. All procedures were performed without anaesthesia by the same operator with Bettochi hysteroscope $\left(\right.$ Storz $\left.^{\circledR}\right)$. Patients received NAIS before procedure. Pain was evaluated with an analogical scale (0-10) during cervical introduction (pain 1) of hysteroscope and tubal placement of Essure ${ }^{\circledR}$ (pain 2). Spearman test was used for correlation between quantitative variables and $\mathrm{T}$ Student test for comparison with quantitative variables.

Results: Six failures (4.2\%) for pain or feasibility were reported. Average pain 2 was significantly higher than pain 1 (3,56+/-2,2 vs 2,73+/-2,41, p: 0.003). Average pains (1 and 2) were not significantly modified by parity or number of vaginal delivery or previous caesarean section $(\mathrm{p}>0.05)$. No correlation was found between pain 1 and parity or number of vaginal delivery, pain 2 and number of vaginal delivery $(\mathrm{p}>0.05)$. Correlation was found between pain 2 and parity (p:0.04).

Discussion: Tolerance of Essure ${ }^{\circledR}$ procedure without anaesthesia is poorly modified by parity or number of vaginal delivery. So, parity and number of vaginal delivery are not helpful to evaluate tolerance before procedure.

Key-words: hysteroscopy, Essure, sterilisation.

\section{P12_12}

Hysteroscopic myoma resection in ambulatory unit: retrospective evaluation

S. Coutinho, O. Lavado

Department of Gynaecology and Obstetrics, da Luz Hospital, Portugal

Summary: Myomectomy by bipolar resectoscope is a safe and efficacious treatment of selected submucous type of symptomatic fibroids. A retrospective clinical assessment of twenty four cases of premenopausal women submitted to resection of benign myomas by hysteroscopy in ambulatory unit is presented.

Introduction: Conservative surgical management of myomas includes myomectomy by hysteroscopy for selected cases of submucous type, that are responsible for significant morbidity in women of reproductive age.

Materials and Methods: Retrospective evaluation of twenty four cases of premenopausal women submitted to resection of benign myomas by hysteroscopy, in ambulatory unit under general or regional anaesthesia, by the same surgeon in private setting, since 2007 until end of 2009. We used a
$9 \mathrm{~mm}$ resectoscope sheath with bipolar loop from Gynecare $^{\circledR}$, with saline distension medium controlled by Endomat $^{\circledR}$ from Storz ${ }^{\circledR}$. Assessment of clinical data, per-operative results and follow-up are presented.

Results: The mean age was 39.9 years (range 31 to 53), ten were nulliparas $(41.7 \%)$ and seven had at least one caesarean section (29.2\%). Twenty one women had metrorrhagia $(83.3 \%)$, eight of them with anemia, and only one case was asymptomatic (4.2\%). At the time of surgery seventeen $(70.8 \%)$ were under medical treatment for control of symptoms. A total of 37 fibroids were removed: sixteen women had one only (66.7\%), and eight (33.3\%) had multiple (between 2 and 4). The Wamsteker classification was: eight $(21.6 \%)$ type 0 , fifteen (40.5\%) type I and fourteen type II $(37.8 \%)$. The medium largest diameter of each fibroid was $17.9 \mathrm{~mm}$ (range 5.0 to 50.0 ). The mean total operating time was 42 minutes (range 20 to 80 ), and the mean fluid balance was $423 \mathrm{~mL}$ (median of $175 \mathrm{~mL}$ ). The resection was done in one time surgery in all cases, and was complete in twenty one of the twenty four cases (87.5\%). Three cases had the following complications, treated expectantly: fluid overload in one case $(>2000 \mathrm{~mL})$, operating time more than 60 minutes in one case, and uterine perforation in one case. The mean follow-up time was 15.4 months (range 3 to 36), with resolution of haemorrhagic symptoms in sixteen of twenty cases $(80 \%)$, and three women with term pregnancies after the surgery. Two cases were submitted to hysterectomy (8.3\%): adenomyosis and multiple myomas were found.

Discussion: In our experience the operating time was comparatively long, and we associate that fact to the type of myoma excised (78.4\% type I or II), and the effort for treating in one time session only. The follow-up is short but until now the results are good, making this technique a safe option for the definitive treatment of selected submucous fibroids.

Key-words: hysteroscopy, submucous myomectomy, ambulatory surgery.

\section{P12 13}

Removal of endometrial polyps by use of Polyp Snares after diagnostic hysteroscopy

C. Grigoriadis, D. Zygouris, G. Derdelis, E. Papakonstantinou, E. Terzakis

$2^{\text {nd }}$ Department of Gynaecology, St. Savvas Hospital, Hellenic Anticancer Institute, Athens, Greece

Our one session hysteroscopic method seems to be safe, easy and successful for diagnosis and removal of endometrial polyps (up to three), using Polyp Snares. The 3.5\% of reoccurrence gives a high therapeutic result. 
Introduction: To present our technique for removal of endometrial polyps by operative hysteroscopy, using Polyp Snares.

Methods: This was a 3-year retrospective study in our Department, performed on 342 patients (35-79 y. mean age 64.5 years). They presented either for symptoms such as menstrual disorders, postmenopausal vaginal bleeding, or with ultrasound findings of endometrial polyps or/and increased postmenopausal thickness of the endometrium. All were one day cases with endometrial polyps which were detected and removed in one session by hysteroscopy. Firstly, diagnostic hysteroscopy (under local anaesthesia, or sedation and in special cases general anaesthesia) was performed. The removal of the polyps was attempted through the working channel by use of NCircle Nitinol Hysteroscopic Polyp Snare (COOK instrument, Fr 5.0, Length $35 \mathrm{~cm}$ ), or Hysteroscopic Duckbill Polyp Snares (COOK instrument, d $15 \mathrm{~mm}$, Fr 5.0, Length $50 \mathrm{~cm}$ ) under direct view. A final hysteroscopy was then performed in order to detect remnants from the base of polyps.

Results: The mean diameter of polyps ranged from 0.4 to $3.5 \mathrm{~cm} .273$ patients presented with one polyp, 43 with two polyps, while 26 with three polyps. 147 patients $(43 \%)$ were symptomatic, while 195 (57\%) were asymptomatic. During the final hysteroscopy we found the raised base of polyps in 39 cases, accounting for a total therapeutic success of $88.6 \%$. Reoccurrence without malignancy was detected into 2 years in 12 cases $(3.5 \%)$.

Discussion: Our method seems to be safe, with sufficient diagnostic-therapeutic outcome, and decreases the possibility of recurrence or undertreatment in comparison with simple curettage.

Key-words: operative hysteroscopy, polyp snare, endometrial polyp.

\section{P12_14}

\section{Quality of sexual life in women who underwent Essure ${ }^{\circledR}$ procedure and outcomes one year later}

A. Agostini, E. Cohen-Solal, M. Petrakian, L. Cravello, M. Gamerre

La Conception Hospital, Marseille, France

Summary: Quality of sexual life was evaluated in patients at the time of Essure ${ }^{\circledR}$ procedure and one year later. Sexual characteristics of women who underwent Essure ${ }^{\circledR}$ procedure were reported. Sexual outcomes at one year were available for a part of this series.

Introduction: Aim of this study was to evaluate sexual characteristics of women who underwent Essure ${ }^{\circledR}$ procedure and sexual outcome one year later.
Materials and Methods: From January 2009 through 1 July 2010, 66 on 80 patients $(82.5 \%)$ who underwent Essure ${ }^{\circledR}$ procedure and 17 on 30 patients $(56 \%)$ contacted one year later accepted to complete the BISF-Q (Brief Index of Sexual Functioning for Women) questionnaire . Quality of sexual life was evaluated with BISF-W questionnaire which provides a quantitative and qualitative assessment of female sexuality according to 7 dimensions: D1 (desire), D2 (arousal), D3 (frequency of sexual activity), D4 (receptiveness), D5 (pleasure, orgasm), D6 (relational satisfaction), D7 (problems affecting sexuality), Composite Score (CS = $\mathrm{D} 1+\mathrm{D} 2+\mathrm{D} 3+\mathrm{D} 4+\mathrm{D} 5+\mathrm{D} 6+07)$. For 30 patients, sexual satisfaction, regret, negative sexual impact and dyspareunia were evaluated one year later.

Results: BISF-Q at the time of Essure ${ }^{\circledR}$ procedure was evaluated: D2 was significantly modified by age $(\mathrm{p}=0.024)$. Previous caesarean section increase significantly D6 $(0.001)$ and D4 $(p=0.034)$. BISF-Q at one year was not yet evaluated because the number of patients was not sufficient. Sexual satisfaction one year later was increased in $24 / 30$ patients $(80 \%)$.No regret, negative sexual impact or dyspareunia was reported one year later.

Discussion: Age and mode of delivery were correlated with quality of sexual life in women who underwent Essure ${ }^{\circledR}$ procedure. Essure ${ }^{\circledR}$ procedure seems to conserve or improve quality of sexual life.

Key-words: Essure, hysteroscopy, quality of sexual life.

\section{P12_15}

Endometrial resection and ablation

F. Colombo, C. Redondo, A. Úbeda

Institut Universitari Dexeus, Barcelona, Spain

Summary: We assessed the clinical results of endometrial resection and ablation (ERA) in our hospital from 1991 to 2008.

Introduction: Heavy menstrual loss is a common health problem in premenopausal women which may cause adverse effects in women's health and quality of life. Hysterectomy has been the standard treatment for women with heavy menstrual bleeding. ERA is a minimally invasive procedure to destroy the endometrium, thus diminishing the menstrual bleeding.

Materials and Methods: Descriptive retrospective study of patients undergoing ERA from January 1991 to December 2008 in the Institut Universitari Dexeus, Barcelona, Spain. 792 women, with an average age of 46.31 years, underwent ERA through the monopolar resectoscope, with loop followed by roller-ball coagulation. Of them, 182 underwent a diagnostic resection and were not included. 
Results: Rates of amenorrhea, treatment success and failure after ERA are described. Two women were reoperated with a second ERA. Operative complications recorded were 23 (3.8\%), (uterine perforation, fluid overload, intraoperative overbleeding and cervical laceration). The probability of amenorrhea after one and 5 years was $80.9 \%$ and $96.5 \%$. The need of hysterectomy increased as time from ERA went by: $1 \%$ after one year and $5 \%$ after five years. During followup, hysterectomy was performed in 34 women (5.5\%), half of them due to clinical conditions non-related to past ERA.

Discussion: ERA offers a less invasive surgical alternative to hysterectomy for the control of excessive uterine bleeding in selected groups of women, in whom other bleeding causes have been ruled out. The shorter hospital stay, little recovery time, low cost and low complications rate make this procedure a good alternative to hysterectomy, with excellent long-term results.

Key-words: hypermenorrhea, endometrial resection and ablation, hysterectomy.

\section{P12_16}

\section{Pregnancy outcomes after hysteroscopic metroplasty as treatment of the uterine septum}

N. Elias, A. Rilo, M. Gracia, R. Nonell, P. Jou, M. Cardona Hospital Clinic de Barcelona, Spain

Summary: We evaluated pregnancy outcomes after a hysteroscopic resection of the uterine septum, divided into two groups depending on the septum size, on all the patients treated in our Department from 2006 to 2008.

Introduction: The septate uterus is the most common congenital uterine malformation and can be associated with recurrent pregnancy loss, preterm labour and infertility.

Materials and Methods: Septate uterus was diagnosed in 29 patients, all of whom were treated by hysteroscopic metroplasty. Data were collected from the operative reports, clinic records and telephone interviews and analized with SPSS v.17. Results: 29 women underwent hysteroscopic metroplasty. 15 of them had a complete septum and 14 had subsepted uterus ( $\leq$ $1 / 2$ uterus). After the treatment 19 women had 28 pregnancies. 12 out of 28 were successful pregnancies ( 8 at term, 2 postterm, 2 preterm, one of them being twins). Only one cesarean delivery was reported.13 ended in miscarriage (10 first trimester, 2 second trimester and 1 ectopic pregnancy) and 3 are currently pregnant. Among the complications of pregnancy we found 2 cervical insufficiencies ( 1 of them ending in second trimester loss and the other one requiring treatment) and one chorioamnionitis in a $25 \mathrm{w}$ twin pregnancy. Discussion: Pregnancy outcomes do not show significant statistical differences between both groups (septum vs subseptum) (Chi square: $p=0.432$ ). Although is not statistically significant $(p=0.159)$, infertility rates show more important decrease after the resection of a complete septum. Further research is necessary.

Key-words: metroplasty, uterine malformation, pregnancy

\section{P12 17}

Hysteroscopy and retained products of conception O. Güell, C. Ros, M. Cardona, R. Nonell, F. Carmona Hospital Clínic i Provincial de Barcelona, Spain

Background: Hysteroscopy permits a direct examination of intrauterine cavity, and nowadays it is a common test for diagnosis and treatment of retained products of conception (RPOC). Some complications and comorbilities are associated with this type of pathology, such as adhesion syndrome, endometritis, hysterectomy, admission at hospital or blood transfusion. Blind curettage has been the classical treatment. However, hysteroscopy is useful as a previous guide of curettage, as a checking after a blind curettage, and as a first-line treatment in some selected patients. Moreover, a direct exam of uterine cavity permits the diagnosis of uterine malformations, which are associated with RPOC in up to $10 \%$ of cases.

Objective: To describe and classify the number of hysteroscopies undertaken in our hospital due to RPOC during the last 3 years.

Results: A total amount of 3026 hysteroscopies have been undertaken in our service between 2007 and 2010. Among them, $19(0.63 \%)$ have been performed as a result of RPOC. Their anatomopathologic results were: retained placental tissue in 8 cases $(0.26 \%)$, placental polyp in 6 cases $(0.20 \%)$ and bone metaplasia in 5 cases $(0.17 \%)$. We noticed two complications after hysteroscopy: one cervical sprain, and one case of metrorrhagia which stopped with a intrauterine Folley catheter.

Conclusions: Besides being a simple, fast and effective technique, histeroscopy shows a safe outcome for the treatment of RPOC.

Key-words: hysteroscopy, retained products of conception, placental polyp.

\section{P12 18}

\section{Atypical polypoid adenomyoma: management} and follow-up

A. Osorio Manyari, R. Nonell Marrugat, P. Jou, J. Ordi, M. Cardona Aparici

Hospital Clinic de Barcelona, Spain 
Summary: There were no endometrial carcinomas in the follow-up of 14 patients with atypical polypoid adenomyomas (APAs). However, $60 \%$ of patients treated by histeroscopic resection had residual or recurrent APA. Introduction: APAs are uncommon $(<150$ reported cases in literature) and usually occurs in premenopausal women. Most cases are benign, although APA has been reported to coexist with or precede the development of endometroid adenocarcinomas of endometrium.

Materials and Methods: Retrospective study:14 patients were diagnosed with APA in our hospital, from 1998 to 2010.

Results: Mean age was 38.1 years. $86 \%$ of subjects were premenopausal. $71 \%$ had abnormal uterine bleeding. Macroscopic features: polyp $50 \%$ and myoma $42 \%$. Initial treatment: 11 hysteroscopic resection and 3 hysterectomy. APA mean size was $18 \mathrm{~mm} ; 66 \%$ located in isthmus. 12 cases of APA and 2 of APA-LMP (low malignant potential). Follow-up: 3-84 months (mean: 26), $6(60 \%)$ residual/recurrent cases (4 APA and 2 APA-LMP). Conservative treatment in 4 cases (mean: 1.5 hysteroscopic resection /patient) and 2 hysterectomy. There were no cases of carcinoma.

Discussion: Hysterectomy is the treatment of choice, but in young women who wish to preserve fertility, local resection and careful postoperative hysteroscopic pathologic surveillance is an acceptable approach, due to high recurrence rate $(30-60 \%)$.The published series indicate an average risk of carcinoma of $8.8 \%$ in patients with APA, although our results do not confirm this fact.

Key-words: atypical polypoid adenomyoma, hysteroscopic resection, hysterectomy.

\section{P12 19}

\section{Hysteroscopic metroplasty treatment of the uterine septum}

A. Rilo, N. Elías, M. Gracia, R. Nonell, P. Jou, M. Cardona Hospital Clínic i Provincial de Barcelona, Spain

Summary: The aim of the study is to evaluate the hysteroscopic metroplasty treatment in septate uterus in all the cases diagnosed in our Gynaecology Department from 2006 to 2008 .

Introduction: Congenital uterine anomalies affect $0.06-10 \%$ of women, and have been associated with poor reproductive outcomes. The most common anomaly is septate uterus.

Materials and Methods: Septate uterus was diagnosed in 29 patients, and all underwent hysteroscopic metroplasty. Data were collected from operative reports, clinic records, and telephone interviews.

Results: 29 women underwent hysteroscopic metroplasty with a mean age of 39.1 years (range 18-40). Before surgery 6 women had 9 pregnancies, all ending in miscarriage. Diagnosis was established by US $(12,41.4 \%)$, hysteroscopy $(4,13.8 \%)$, $\operatorname{HSG}(8,27.6 \%)$, RMI $(2,6.9 \%)$ and US-HSG $(3,10.3 \%)$. We also evaluated the septum size: 10 complete septum, 8 septum $1 / 3$ of the uterus, $6 \frac{1 / 2}{2}$ of the uterus, and $52 / 3$ of the uterus. 6 patients $(20.7 \%)$ needed a second surgery to get a normal endometrial cavity. Surgery was in all cases under general anaesthesia. Resection was performed with resectoscope with continuous glicine flow. No surgical complications were reported. After surgery, 4 patients (13.8\%) did not achieve pregnancy, 18 (62.1\%) achieved spontaneous pregnancy, and 4 (13.8\%) got pregnant by fertility treatments.

Discussion: Hysteroscopic septum resection is a feasible and effective procedure, with low morbidity, and improves pregnancy rates after surgery.

Key-words: metroplasty, septate uterus, hysteroscopic results.

\section{P12 20}

\section{Do the results improve with the use of monopolar endometrial resection-ablation concomitant with hysteroscopic submucosal myomectomy for the treatment of menorrhagia?}

B. Romero Guadix, A.J. Rodriguez Oliver, J. Fernandez Parra, A. Gonzalez Paredes, T. Aguilar, F. Montoya Servicio de Obstetricia y Ginecologia. Hospital Virgen de las Nieves de Granada, Spain

Summary: Hysteroscopic myomectomy must be combined with monopolar endometrial resection-ablation (ERA) to improve results in the control of menorrhagia in premenopausal patients without fertility desires.

Introduction: To evaluate the effect of hysteroscopic myomectomy on the outcome of premenopausal patients undergoing monopolar endometrial resection-ablation for menorrhagia or menometrorrhagia.

Materials and Methods: Retrospective cohort study of 260 women underwent monopolar endometrial resectionablation. In 50 of them (19.2\%) who had submucosus myoma a concomitant myomectomy was done.

Results: The mean follow-up period was 30 months. Bleeding was controlled in $82.9 \%$ of patients with endometrial resection-ablation and in $92 \%$ of patients with a concomitant myomectomy $(\mathrm{p}<0.05) .5$ patients in the group of myomectomy and ERA $(10 \%)$ and 27 in the group of ERA alone (12.8\%) needed another type of surgery, hysterectomy the most usual.

Discussion: Hysteroscopic myomectomy at the time of monopolar endometrial resection-ablation improves results in the control of menorrhagia, probably because the myoma presence is the main cause of bleeding. 
Key-words: hysteroscopic myomectomy, endometrial resection-ablation, results.

\section{P12 21}

\section{Complications of hysteroscopy; retrospective study in Hospital Clinic de Barcelona}

P. Sokol, M. Cardona Aparici, P. Jou Collell, R. Nonell Marrugat, F. Carmona Herrera

Hospital Clinic de Barcelona, ICGON, Spain

Summary: The aim of this study was to estimate rate of hysteroscopy-related complications. Data was obtained from all hysteroscopic procedures from last 3 years. Complication rate from all the procedures was $0.99 \%$. Diagnostic procedures had lower complication rate than operative procedures. Introduction: The aim of this study was to estimate the incidence of complications of diagnostic and operative hysteroscopic procedures in our centre and to describe their nature.

Materials and Methods: Data on complications was recorded in our centre from last 3 years. We took into consideration all patients submitted to diagnostic or operative hysteroscopy that took place in outpatient clinic or operating theatre.

Results: 30 complications occurred among 3044 hysteroscopic procedures (rate $0.99 \%$ ). Diagnostic procedures had 57 -fold lower complication rate $(0.05 \%)$ than operative procedures (rate $2.85 \%$ ), $\mathrm{p}<0.05$. Operative hysteroscopies performed in outpatient clinic had 10-fold lower complication rate $(0.4 \%)$ than procedures carried out in operating theatre $(4.1 \%), p<0.05$. The most frequent surgical complications were post-discharge visits in emergency unit that occurred in 7 cases (rate $0.7 \%$ ) and fluid overload of distension medium that happened in 5 cases (rate $0.5 \%$ ).

Discussion: Diagnostic hysteroscopies had very low complication rate, indicating that they are safe procedures to evaluate intrauterine pathology. The incidence of complications was lower among operations carried out in outpatient clinic than in the operating theatre. The rate of complications of operative hysteroscopies from our study was similar to rates described in recent prospective studies. Key-words: hysteroscopy, complications, operative.

\section{P12_22}

The causes of intrauterin adhesions and the outcomes after hysteroscopic treatment

T. Var, E. Tonguc, A. Cit1l, S. Batioglu

Zekai Tahir Burak Women Health Education and Resarch Hospital, Ankara, Turkey
Introduction: The aim of this study is to evaluate prediposing factors of intrauterine adhesions and efficacy of hysteroscopic adesiolysis on re-establisment of the uterine cavity, postoperative resumption of menses and pregnancy rate.

Materials and Methods: Retrospectively, 124 patients with intrauterine adhesions were enrolled in the study at Zekai Tahir Burak Women's Health Resarch and Education Hospital between 2004-2008. All cases were hysteroscopically scored according to AFS Classification. Hysteroscopic adhesiolysis was done in the early proliferative phase of menstrual cycle in the patients who were menstruating.

Results: Of 124 patients, 28 patients (22\%) with Stage 1 intrauterine adhesions,54(44\%) Stage 2 and 42(34\%) Stage 3. Of 124 patients, 76 of them had reproductive problems and other 48 patients had only menstrual abnormality. The menstrual disorders $\%$ in stage 3 and infertility $71 \%$ stage 1 . The main prediposing factor was pregnancy related curratage (70\%). Menstrual disorders (amenorrhea and hypomenorrhea) were seen in patients with severe adhesions (73\%). The rate of amenorrhea before adhesiolysis was $\% 26$ and after $7 \%$. $(p=0.01)$. The pregnancy rate was $48.5 \%(37 / 76)$ and the live birth rate was $36.8 \%(28 / 76)$.

Conclusions: Pregnancy related curratage was the most common factor for intrauterine adhesions, especially for severe adhesions.

Key-words: causes of intrauterin adhesions, hysteroscopic treatment outcome, Asherman syndrome.

\section{Poster Session 13_Operative Risk Management}

\section{P13_1}

\section{Conservative versus laparoscopic management} of ectopic pregnancy

C. Christodoulaki, G. Stathoydakis, E. Ziogos, I. Zouraraki, G. Georgopoulou, M. Neonaki, I. Matalliotakis Department of Obstetrics and Gynaecology, University School of Medicine, Crete, Greece

Materials and Methods: Between 2005 and 2009 a total of 123 patients were hospitalized for ectopic pregancy in our department. Inclusion criteria for conservative treatment with methotrexate (MTX) were: (i) pre-operative serum HCG $3000 \mathrm{IU} / 1$; (ii) ectopic gestation , $4 \mathrm{~cm}$ in diameter; (iii) no rupture of the tube, and (iv) normal liver and renal function. No restrictions were required for laparoscopic surgery. Although 70 satisfied the criteria for conservative treatment, 38 agree to follow the protocol of variable dose of methotrexate. Ten of these 
patients were operated because of tubal rupture. Eighty five patients were operated laparoscopically. Laparoscopic surgery has been reported to have several short-term benefits, such as shorter hospitalization times, shorter convalescence periods and less complications in comparison with methotrexate .

Conclusion: laparoscopic surgery has proved to be more economical and more efficient treatment for ectopic pregnancy.

Key words: methotrexate, ectopic pregnancy, complications.

\section{P13 2}

Patient safety risk factors in MIS: ESGE Expert's opinion

S.P. Rodrigues, F.-W. Jansen

Leiden University Medical Center, The Netherlands

Objective: To determine the weights of patient safety (PS) risk factors in MIS.

Material and Methods: Eight PS risk factor domains were identified with a literature research and a questionnaire was drafted containing 34 questions based on a 13 point Likert scale. The questionnaire was critically reviewed on clinical relevance and completeness (consensus validity) by three experts in the field of PS (two on PS in MIS and one on PS in general). It was distributed among known PS experts during the 2009 ESGE congress in Florence and afterwards also sent electronically to internationally recognized PS experts.

Results: A total of 41 questionnaires were distributed and the response rate was $71 \%$. Mean scores of the risk factor domains showed that the surgeon's knowledge and MIS skills were the most influencing factors (11.5), followed by technology related items (11.0). Further rating was: social interaction 10.3, outcome of the surgical procedure 9.7, fallibility of the surgeon 9.1, general functioning of OR team members 9.0, patient related risk factors (ASA, BMI) 8.3, safety measures 8.2 and distractions 7.2.

Conclusions: The results indicate that all items are of importance to patient safety; however the responsibility of the MIS surgeon is stressed. Experience of the surgeon, his technical operative skills and knowledge about how to handle laparoscopic equipment are generally rated as most important factors for PS in MIS. These items are closely followed by good functioning of instruments and equipment. Therefore it can be concluded that a laparoscopic training program should, besides focusing on operative skills, also include proper training in management of laparoscopic operating units.

Key-words: Patient safety, risk factors, Expert opinion.

\section{P13 3}

Management of ovarian dermoid cysts by laparoscopy compared with laparotomy in the University Hospital of Crete (Greece)

G. Stathoydakis, C. Christodoulaki, E. Ziogos, I. Zouraraki, N. Stefanidoy, M. Neonaki, I. Matalliotakis

Department of Obstetrics and Gynaecology, University School of Medicine, Crete, Greece

Methods: Sixteen patients with ovarian dermoid cysts removed by laparoscopic surgery were compared with 30 patients with ovarian dermoid cysts removed by laparotomy, with respect to the selection criteria, surgical procedures, operating time, intraoperative and postoperative complications, blood loss and hospital stay.

Conclusion: The operating time performed by laparoscopic surgery was longer $(60+12 \mathrm{~min}$, mean $+\mathrm{SD})$ than those for the same procedures performed by laparotomy $(30+15 \mathrm{~min}$, $\mathrm{p}<0.01$ ), At the end of this study, the times taken for laparoscopic procedures were almost the same as those for laparotomy. Less blood loss $(12+0.7 \mathrm{ml}$ versus $85.6+$ $13.4 \mathrm{ml}, \mathrm{p}<0.01)$ and shorter hospital stay $(2+1$ days versus $4+2$ days, $p<0.01$ ) were the advantages of laparoscopic surgery found at the end of this study. The efficiency and safety of operative laparoscopy as an alternative access route for the management of ovarian dermoid cysts were recognized.

Key-words: dermoid cyst, intraoperative complications , postoperative complications, blood loss.

\section{P13 4}

Perinatal and surgery outcomes in adnexal mass during pregnancy

M.C. Merced, E. Suárez, O. Puig, B. Díaz, S. Manrique, J. Xercavins

Vall Hebron Hospital, Barcelona, Spain

Introduction: Ovarian tumors are estimated to occur in about 1 in 1000 pregnancies and 3\% are malignant. The aim of the study was to evaluate the safety and perinatal outcomes of laparoscopic approach in the management of adnexal mass during pregnancy.

Methods and Procedures: Retrospective study. We included 7 pregnant women, during the first $(1 / 7)$ or second trimester (6/7) of pregnancy from January 2008 to March 2010 in Vall Hebron Hospital. In all cases the surgery was performed for pelvic pain, suspicious mass or persistent big mass. The surgery performed was laparoscopic salpingoophorectomy or ovarian cystec- 
tomy. Closed technique was used (Verres needle in the Palmer's point). The remaining trocars were placed under direct vision on different areas of the abdomen depending on the size of the uterus and adnexal mass. Results: Gestational age at surgery varied from 8 to 21 weeks and the mass size was 60 to $126 \mathrm{~mm}$. The salpingo-oophorectomy was performed in $6 / 7$ patients and the ovarian cystectomy in $1 / 7$. No conversions to open surgery. There were no intra or post operative complications. The majority of patients were discharged home on the second postoperative day. Pathologic exams showed: 2/7 endometrioma, 2/7 teratoma, 2/7 cystadenoma mucinosum and 1/7 massive necrosis. Perinatal outcomes were: 1/7 chorioamnionitis, $1 / 7$ induction at $36 \mathrm{w}$ by polyhydramnios, $3 / 7$ at term birth without complications and $2 / 7$ even pregnant.

Discussion: Laparoscopic surgery in pregnancy is safe and carries a low morbidity. Although most authors use open laparoscopy, the closed technique with security manoeuvers is safe in these patients. There were no adverse effects on perinatal outcomes with laparoscopic surgery.

Key-words: adnexal mass, pregnancy, laparoscopic approach.

\section{Poster Session 14_Robotics}

\section{P14_1}

Initial experiences of robot-assisted laparoscopic surgery for the benign and malignant gynaecologic diseases

S.-h. Park

Department of Obstetrics and Gynaecology, Hallym University, Korea

Objective: To evaluate surgical outcomes, feasibility and safety of robot-assisted laparoscopic surgery using the da Vinci robotic system.

Methods: Twenty-seven patients underwent various gynaecologic robot-assisted laparoscopic surgery using da Vinci robotic system between September 2007 and December 2009 , and were assessed for surgical outcomes. This study was carried out retrospectively in the department of obstetrics and gynaecology at Hallym University, Seoul, Korea. The main indications for operation were cervical cancer $(n=13)$, followed by endometrial cancer $(n=8)$, myoma $(\mathrm{n}=6)$.

Results: All patients successfully underwent various robotassisted laparoscopic surgery such as radical hysterectomy, hysterectomy, myomectomy. The mean operating time was $175.6 \pm 49.2$ minutes, the mean hemoglobin change and estimated blood loss were $1.7 \pm 0.7 \mathrm{~g} / \mathrm{dL}$ and $33.1 \pm 24.4 \mathrm{~mL}$ respectively. The mean time from skin incision to docking the robot was $12.4 \pm 8.2$ minutes and the mean console time was $108 \pm 59.3$ minutes. The length of hospital stay averaged $4.9 \pm 1.4$ days. No complications requiring treatment occurred.

Conclusion: Robot-assisted laparoscopic surgery using the da Vinci robotic system is a feasible and safe treatment modality in the benign and malignant gynecologic diseases. Key-words: robot-assisted laparoscopic surgery, the da Vinci robotic system, benign and malignant gynecologic diseases.

\section{Poster Session 15_Single Access Surgery}

\section{P15_1}

\section{Results of Single-Port Laparoscopy in gynaecology in Ukraine}

S. Angioni ${ }^{\circ}$, N. Yarotsky, V. Lischuk, N. Mendel, A. Efimenco

National O.O. Bohomolets Medical University, Obstetrics and Gynaecology Department, Universal clinic "Oberig", Ukraine; 'Division of Gynaecology, Obstetrics and Pathophysiology of Human Reproduction, University of Cagliari, Italy

Summary: This topic describes the results of single-port laparoscopy in gynaecology for treatment of such pathologies as endometriomas and persistent functional cysts of the ovaries, as well as it allow getting an excellent cosmetic effect in late postoperative period and high satisfaction of the patients.

Introduction: in December 2009 during the international conference "New face of gynaecological surgery" in memory of Dr. Efimenco, that took place on the basis of "Oberig" clinic, laparoscopic surgery from single transumbilical access was performed on pelvic organs.

Material and Methods: Our patients were operated with Karl Storz Single port X-cone equipment for such pathologies as endometriomas and persistent functional cysts of the ovaries, the diagnoses were confirmed by histological testing. Duration of the operation was average $50 \mathrm{~min}$. Patients were discharged 24 hours after surgery, without any complications. All of them were examined 3 months after surgery, and the result showed high efficiency of surgical treatment and good cosmetic effect.

Results: This topic showed high efficiency of single-port laparoscopy in gynaecology combined with good cosmetic result that satisfied the patients. 
Key-words: single-port laparoscopy, gynaecology, cosmetic result.

\section{P15 2}

\section{Comparative study between SPA-LAVH and conventional 3 Port LAVH in patients with CIS of uterine cervix} W.-D. Joo, Y.-i. Ji, H.-J. Roh, S.-J. Lee, H.-J. Yoo Department of Obstetrics and Gynaecology, Ulsan University Hospital, University of Ulsan College of Medicine, South Korea

Background and objectives: We investigated the feasibility of single port access laparoscopic-assisted vaginal hysterectomy (SPA-LAVH) in patients with carcinoma in situ (CIS) of uterine cervix, whose uterus is not enlarged hence it is easy to handle compared to uterine fibroid and adenomyosis.

Methods and Procedures: Through a $3 \mathrm{~cm}$ vertical incision within umbilicus, Alexis ${ }^{\circledR}$ wound protector was inserted. A surgical glove wrapped the wound retractor and three $5 \mathrm{~mm}$ trochars were inserted into the fingers of the glove for a $5 \mathrm{~mm}$ laparoscope, EnSeal ${ }^{\circledR}$ tissue sealing device (Ethicon Endo-Surgery Inc.) or a suction irrigator, and a Real-hand ${ }^{\circledR}$ forceps. LAVH was performed in the same manner with conventional three port LAVH (TP-LAVH) and uterine manipulator was extensively used like one of laparoscopic forceps. We performed 15 cases of SPA-LAVH between April 2009 and January 2010. We also enrolled 33 women with CIS of cervix who received TP-LAVH between March 2006 and April 2009 as a control group. We compared demographic factors and outcomes of surgery between two groups.

Results: There were no significant difference between SPALAVH group and TP-LAVH group in age (mean $45.40 \pm$ 9.55 years vs. $46.75 \pm 10.23$ years), body mass index (mean $24.52 \pm 4.19$ vs. $24.08 \pm 3.35$ ), parity (mean $2.40 \pm 1.05$ vs. $2.45 \pm 1.14$ ), previous surgery (mean $0.33 \pm 0.61$ times vs. $0.36 \pm 0.96$ times), hemoglobin change (mean $1.51 \pm 0.61 \mathrm{~g} / \mathrm{dl}$ vs. $1.66 \pm 0.96 \mathrm{~g} / \mathrm{dl}$ ) and complications (0 vs. 2). SPA-LAVH group took a longer time during surgery (103.80 \pm 13.3 minute vs. 91.21 \pm 27.69 minutes; $p=0.007)$ and stayed shorter in hospital (2.66 \pm 0.72 days vs. $3.27 \pm 0.94$ days; $\mathrm{p}=0.029)$.

Conclusions: SPA-LAVH is feasible for the patients with CIS of uterine cervix.

Key-words: Single-port access, hysterectomy, carcinoma in situ of uterine cervix.

\section{P15 3}

\author{
Single-port and two-port laparoscopic hysterectomy \\ and adenxal surgery \\ J.-Y. Park, D.-Y. Kim, J.-H. Kim, Y.-M. Kim, Y.-T. Kim, \\ J.-H. Nam \\ Department of Obstetrics and Gynaecology, University of \\ Ulsan College of Medicine, Asan Medical Center, Republic \\ of Korea
}

Introduction: The aim of this study was to estimate the feasibility, safety and surgical outomes of single-port and two-port laparoscopic hysterectomy and adnexal surgery. Methods: Single-port hysterectomy $(\mathrm{n}=50)$ and adnexal surgery $(n=41)$ was performed through a $2-\mathrm{cm}$ intraumbilical incisions using wound retractor and surgical glove. For two-port hysterectomy $(n=153)$ and adnexal surgery $(\mathrm{n}=143), 1.5-\mathrm{cm}$ intraumbilical incision was made and 5-mm ancillary port were inserted on suprapubic area. Results: In single-port hysterectomy group, the mean uterine weight, operating time, estimated blood loss (EBL), and postoperative hospital stay were $225 \mathrm{gm}$ (range, 61-490 gm), $132 \mathrm{~min}$ (range, 70-244 min), $99 \mathrm{~mL}$ (range, 10-300 $\mathrm{mL}$ ), and 2 days (range, 1-4 days) respectively. Transfusion was required in two patients and perioperative complications occurred in 2 patients ( 1 bowel injury and 1 bladder injury). In single-port adnexal surgery group, 9 underwent bilateral salpingo-oophorectomy ( $\mathrm{SO}$ ), 5 underwent unilateral $\mathrm{SO}, 4$ underwent bilateral ovarian cystectomy (OC), and 23 underwent unilateral OC. The mean size of ovarian tumor, operating time, estimated blood loss (EBL), and postoperative hospital stay were $6 \mathrm{~cm}$ (range, 2$15 \mathrm{~cm}$ ), $95 \mathrm{~min}$ (range, 59-183 $\mathrm{min}$ ), $76 \mathrm{~mL}$ (range, 10$200 \mathrm{~mL}$ ), and 2 days (range, 1-4 days) respectively. There was no perioperative transfusion requirement and complication. In two-port hysterectomy group, the mean uterine weight, operating time, estimated blood loss (EBL), and postoperative hospital stay were 293 gm (range, 49$1220 \mathrm{gm}$ ), $115 \mathrm{~min}$ (range, 61-258 $\mathrm{min}$ ), $142 \mathrm{~mL}$ (range, 20-500 $\mathrm{mL}$ ), and 2.8 days (range, 1-8 days) respectively. Transfusion was required in three patients and perioperative complications occurred in 7 patients ( 2 bladder injuries and 5 postoperative febrile morbidities). In two-port adnexal surgery group, 15 underwent bilateral SO, 25 underwent unilateral SO, 20 underwent bilateral $\mathrm{OC}$, and 83 underwent unilateral OC. The mean size of ovarian tumor, operating time, estimated blood loss (EBL), and postoperative hospital stay were $5.7 \mathrm{~cm}$ (range, 2-20 cm), $86 \mathrm{~min}$ (range, 41-261 $\mathrm{min}$ ), $81 \mathrm{~mL}$ (range, 10-300 $\mathrm{mL}$ ), and 2.4 days (range, 1-7 days) respectively. There was no 
perioperative transfusion requirement. One patient suffered from postoperative ileus.

Conclusion(s): Single-port and two-port laparoscopic hysterectomy and adenxal surgery were feasible and safe alternatives to conventional three or four port laparoscopic surgery in selected patients.

Key-words: Sing-port surgery, two-port surgery, gynaecologic surgery.

\section{P15 4}

\section{Total laparoscopic hysterectomy with Single-Port} Access: report of 10 cases

E. Ratia Garcia, C. Rigau, L. Cusiné, J. Rovira, J.C. Mateu, M. Degollada, P. Brescó

Hospital of Igualada, Spain

To present our initial experience with single port-access laparoscopic hysterectomy in 10 women with benign uterus pathology in Igualada's Hospital from January 2010 to June 2010. The first laparoscopic hysterectomy was reported by Reich et al. in 1989. In the 1960s, Wheeless and Thompson reported on more than 4000 women who underwent rapid, inexpensive and effective surgical sterilization at single-trocar laparoscopy. In 1991, Pelosi and Pelosi performed the first complex procedure using a single-trocar technique, a supracervical hysterectomy. From January 2010 to June 2010, laparoendoscopic single-site surgery (LESS) total hysterectomy was performed in 10 pre-menopausical patients diagnosed with a variety of benign pathology: menometrorrhagia, cronic pain and complex hyperplasia with atypia. Demographics and surgical outcomes: age (y): X=45 (41-49), BMI (kg/m2): $X=26,7$ (19,9-33-9), operative time (min): $X=92(60-110)$, estimated blood loss ( $\mathrm{Hb}$ pre-Hb post): $\mathrm{X}=1,4 \quad(0,9-2,0)$, weight of uterus (gr): $X=139$ (64-298). Postoperative hospital stay (d): $X=3$ (2-7). There were no operative complications. One case of paralytic ileo increased time spent in a hospital by 7 days. LESS is an alternative to conventional multiport laparoscopy. The advantages of Singleaccess laparoscopy using a transumbilical port may include less infection and hernia formation and better cosmetic outcome. The disadvantages and limitations include longer surgery time, difficulty in learning the technique, and need for specialized instruments. The single-access technique may be difficult in a large uterus and in patients with adhesions and endometriosis.

Key-words: Single-Port Access, hysterectomy, laparoscopy.
Poster Session 16_Teaching \& Training

\section{P16 1}

Assessment of ovarian biopsy

M. Panevska-Gareva, K. Meinhard, R. Zhivkova

University Hospital of Ob\&Gyne "Maichin dom" Sofia, Bulgaria

One of the most frequently encountered cause of female infertility is PCOS.

Objective: Various methods have been proposed and are currently used in the assessment of ovarian reserve in order to predict the outcome for pregnancy. Female age, basal follicle stimulating hormone/luteinas

ing hormone ratio, basal estradiol, inhibin-B levels are classical analyses for PCOS patient. We evaluate the accuracy of histological findings and results of diagnostic methods to detect structural chromosomal abnormalities and aneuploidies (FISH -fluorescence in situ hybridization). Materials and Methods: A prospective investigation of 74 patients with PCOS and infertility was undertaken for 3 years period. All patients had laparoscopic surgery-biopsy of the ovaries and drilling. Ovarian biopsy samples we devided in two parts. One piece of sample was refered to the histological laboratory and the other piece of sample - to the Department of Biology for FISH. The patients were devided in four groups depending on female age (1st group: 31 patients - from 20 to 26 years old; 2 nd group: 26 patients - from 27 to 33 years old; $3^{\text {rd }}$ group: 11 patients - from 33 to 39 years old; 4th group: 6 patients - more 40 years old).

Results: Microscopic findigs from ovarian biopsy in 74 patients with PCOS were the following: the superficial cortex is fibrotic and hypocellular. There are primordial follicles and they are normal in number and appiarance. The next findings were: the superficiale cortex is fibrotic, the stroma is fibrotic, absent primordial follicles. The next findings: stigmata of ovulatio, like corpora albicantia. The last one: the superficial cortex is fibrotic with hypocellular stroma, atretic cystic follicles and primordial follicles. We received the results from 18 materials, investigated by FISH method.

Conclusion: The results of our investigation is very diificult to analyze. The ovary probably change own characteristics after drilling-ovarian metabolism and physiology are restored and this changes our clinical prognosis for pregnancies. The controversial results among investigated patients with present and absent primordial follicles could be explainded with the size of 
pieces and the field of biopsy. While the mechanism of surgery on the ovaries restore ovulation is unclear we will continue to work for the treatment of PCOS -related infertility.

Key-words: PCOS, ovarian biopsy, laparoscopy.

\section{P16 2}

\section{Laparoscopic management of tubal pregnancy: how effective is the training in the UK? \\ R. Bharathan, S. Meritte, H. Ahmed \\ Medway Maritime Hospital, Kent, UK}

Competency in laparoscopic management of tubal pregnancy is mandatory for the completion of training in the $U$. $\mathrm{K}$. This study explores the self-assessed experience of the newly qualified consultants, their perspective of their training and potential impact of new measures. A postal questionnaire of newly qualified consultants was performed in 2008. Response rate was $37 \%$ amongst 135 candidates. Mean duration of training was 9.6 years. During the final 12 months of training, the median number of procedures they were involved in was 10 , and the median number of procedures where they performed most of the surgery was 6. $14 \%$ said they had not reached independent competency at certification. $60 \%$ performed salpingotomy when suitable and $60 \%$ preferred diathermy excision. $92 \%$ of respondents had attended a relevant course. $43 \%$ reported access to a simulator. The mean Likert score on a 5 point scale regarding satisfaction of training was 4.2. Continued professional development is the template that promotes seamless progress through lifelong learning. The $14 \%$ of respondents who did not reach competency at certification, continued to receive support from senior colleagues during their transition. Increased presence of consultants, recently introduced specific skills modules and objective structured assessment of technical skills should enhance training. Diathermy excision is associated with more pain, longer surgery and increased hospital stay than endoloop. The learning curve for salpingotomy is longer than that of salpingectomy. Addressing these factors along with diligent use of simulators is essential for completion of training and maintaining skills.

Key-words: Tubal pregnancy, laparoscopic surgery, training.

\section{P16_3}

Laparoscopic training program of Igualada hospital J. Rovira, L. Cusiné, I. Aznar, C. Rigau, E. Ratia, J.C. Mateu, P. Brescó

Hospital of Igualada, Spain
Introduction: Over the last few decades, minimally invasive techniques, such as laparoscopy, have been increased. In our speciality laparoscopy is taking a leading role and it is becoming the standard approach for many conditions. It has advantages such as less surgical trauma, faster postoperative recovery and reduce the postoperative pain and stay. However, there are disadvantages as large investment in instruments, long and costly training period, longer operation time and higher rate of surgical complications during the learning curve. The practice with corpses, life animals o virtual simulators are expensive alternatives. The utilization of pelvitrainers are an efficient option, applicable in Centres with novices.

Project: The International Laparoscopic Gynaecology School (EIDEG) was founded in 2006 by Igualada, Mútua de Terrassa and Clinic of Barcelona Hospitals with the purpose of helping the professionals who begin and those who want to improve their knowledge and skills. The need of learning of junior surgeons and residents of Igualada Hospital, led us to design a Training Program Department together with EIDEG.

Training Program: This program is a voluntary activity at the headquarters School, under the supervision of the Head Department. The training program includes:

- surgical anatomy classes,

- pelvitrainer exercises to approach the management of surgical instrumental, to adapt two-dimensional image and to improve surgical perception,

- exercise for dissection, cut and sutures and the learning of knots,

- visualize experts surgeons videos and self criticism surgeries carried out by pupils.

Conclusions: Nowadays, the best method to teach the laparoscopy is not defined but the literature show that the use of simulators are very useful to learn basic techniques and allow the improvement of surgeon skills and the coordination. Therefore, simulators shoud be implemented in the educational program. The instructor to guide and to evaluate the pupils is a basic figure to get the correct use and the maximum performance of these methods. Not all the Services can have simulators, so we must promote the creation of laparoscopy formation schools as ours, EIDEG.

Key words: Pelvitrainer, training, gynaecology laparoscopy

\section{P16_4}

Endoscopic intracorporal knotting and suture techniques: talent or experience?

C. Banerjee, M. Cosentino, W. Hatzmann, K.G. Noé

Hospital Dormagen, Cologne, Germany 
Introduction: Endoscopic intracorporal knotting and suture techniques demand sophisticated surgical skill. Young surgeons can be trained to perform endoscopic intracorporal techniques using dummies. This study examines whether talent or practice is more likely to predict how well an endoscopic surgeon performs endoscopic intracorporal techniques. Coexisting factors including sinistrality and myopia were sub-analysed.

Materials and Methods: 25 medical students participated in an endoscopic suture teaching program (ESTP). The program included two three-hour sessions of intensive training at the pelvic trainer. In the first session, each student executed three complete knots followed by one continuous suture that starts and ends with a knot. During the second session (one week later) the students completed a single knot followed by two continuous sutures (two knots). Each complete knot consists of a double knot in one direction and a single knot in the reverse direction. Each student's skill level was determined by measuring their knotting times. Individual factors such as size, weight, length, gender, age, myomia, sinistrality etc., and education, including previous hours of laparoscopic assistance, industrial arts and co-factors (sports, music, were accounted for with a selfadministered questionnaire.

Results: No correlation between individual and co-factors was found and there was no significant difference between the performance of right and left handed individuals. Students with more than 20 hours of experience assisting endoscopic operations had significantly faster knotting times for the first three knots than students who had less than 20 hours or no experience assisting endoscopic operations. Conclusion: The development of the skills required to perform endoscopic knotting and suture techniques can be attributed to experience rather than talent.

Key-words: knotting technique, pelvi trainer, talent.

\section{Poster Session 17_Technical Tricks and New Instrumentations}

\section{P17_1}

\section{Laparoscopic treatment of ovarian dermoid cyst. Factors related to intraoperative spillage, clinical significance and results}

A.J. Rodríguez-Oliver, M.T. Aguilar Romero, M.P. Carrillo Badillo, J. Fernández Parra, A. González Parede, F. Montoya Ventoso

Servicio de Obstetricia y Ginecologia, Hospital Virgen de las Nieves, Granada, Spain
Summary: The laparoscopic surgery is the first treatment option in the management of ovarian dermoid cysts. There are few complications and the healing is rapid. There are no consecuences, generally, if spillage ocurrs.

Introduction: The aim of this study was to analyze the surgical characteristics of the laparoscopic treatment of dermoid cyst.

Material and Methods: A retrospective research was done. One hundred and thirty-one dermoid cysts were treated by laparoscopy at Virgen de las Nieves, University Hospital from January 2000 to December 2008.

Results: The mean age of the patients was 32.4 years. The mean size of the cyst was $63.8 \mathrm{~mm}$. In $62.6 \%$ of the cases was possible to do conservative surgery (cystectomy). Only two patients needed reintervention due to bleeding complications. The mean of incoming was 1.6 days. The intraoperative spillage occurred more commonly when cystectomy was done. No case of peritonitis was reported.

Discussion: Laparoscopic trreatment of ovarian dermoid cyst has peculiar characteristics due to its tissular composition. It has been promoted a special management to avoid spillage, but most of studies didn't show any significance to this fact.

Key-words: ovarian dermoid cyst, laparoscopic surgery, intraoperative spillage

\section{P17_2}

\section{A modified intracorporeal knot-tying technique using an endoknot cannula during laparoscopy: coiling and snaring method}

S.W. Yi ${ }^{1}$, S.M. Park ${ }^{2}$

${ }^{1}$ Department of Obstetrics and Gynaecology, Gangneung Asan Hospital, University of Ulsan College of Medicine, ${ }^{2}$ Knowledge \& Information Design Innovation Center, Gangwon University - South Korea

Introduction: Laparoscopic suturing and tying technique is an essential skill for a surgeon performing laparoscopic surgery. Despite advances made in the fields of optics and imaging, the procedure is difficult and requires laparoscopists to be trained. Especially in single-port or two-port surgical technique, the surgical instruments have a limited motion range, which makes knot tying more difficult than in conventional laparoscopy. Here, we present a safe and easy intracorporeal knotting technique.

Materials and Methods: An Endoknot or Vicryl 1.0 suture material inserted ino an endoknot cannula was used. After positioning the needle, it is grasped with a needle holder or needle block. The suture is inserted through the tissue. Two or three loops are formed with the endoknot cannula, 
and the unlocking of the loops is prevented by compressing it with the graspers (coiling). The formed loops are snared on the grasper, and the free end of the suture is held by the graspers and brought inside the formed loops (snaring). The suture is tied over the tissue using the graspers, and the cannula acts as an integral knot pusher. Additional knots are applied over the suture by using the same method.

Results: Our modified intracorporeal knot-tying is simple, safe, and fast to create.

Discussion: Our modified technique for intracorporeal knot tying makes laparoscopic suturing feasible, especially when the angle between the hand instruments is not ideal and the working space is limited.

Key-words: intracorporeal knot tying, endoknot, laparoscopy.

\section{P17_3}

Transumbilical retrieval of surgical specimens through a multichannel port during single-port or 2-port laparoscopy for the removal of adnexal masses S.W. Yi, S.S. Lee, D.H. Ju, W.S. Sohn

Division of Minimally Invasive Surgery \& Gynaecologic Laparoendoscopy, Department of Obstetrics and Gynaecology, Gangneung Asan Hospital, University of Ulsan College of Medicine, South Korea

Introduction: Laparoscopy has been used for the removal of many adnexal masses. With a conventional 5- or $10-\mathrm{mm}$ port, it is a difficult, time-consuming procedure to retrieve surgical specimens, especially in large size of cystic masses. Therefore, we previously introduced a laparoscopic technique in which a multichannel port is used. This study aimed to evaluate the effectiveness of a multichannel port by using a wound retractor for the retrieval of surgical specimens during single-port or 2-port laparoscopy for the removal of adnexal masses.

Materials and Methods: A retrospective observational study was performed on 278 patients who underwent laparoscopy for the removal of adnexal masses from November 2006 to May 2010, and the medical records, including videos obtained during the operation, were reviewed. Patients in whom laparoscopy was performed using the multichannel port (multichannel port group; $\mathrm{n}=66$ ) were compared with those who underwent conventional laparoscopy (conventional laparoscopy group; $\mathrm{n}=212$ ), and the differences were statistically evaluated.

Results: All specimen-retrieval procedures performed using the multichannel port were successfully carried out. There were no intraoperative complications or rupture of the endoscopic bag. The mean mass size was $7.2 \pm 3.8 \mathrm{~cm}$ in the multichannel port group and $6.1 \pm 2.6 \mathrm{~cm}$ in the conventional laparoscopy group. The mean time required for the retrieval procedure was shorter $(2.3 \pm 1.9$ minutes $)$ in the multichannel port group than in the conventional laparoscopy group (2.8 \pm 3.8 minutes). Despite the larger wound caused by the multichannel port, the postoperative wound scar was minimal and cosmetically acceptable because it was concealed in the umbilicus, unlike the scar caused by conventional laparoscopy, which was in the iliac fossa.

Discussion: Transumbilical retrieval of surgical specimens through a multichannel port by using a wound retractor during laparoscopy is a safe procedure, and it reduces the operation time by decreasing the time required for specimen retrieval through a small port site. This technique also seems to be cosmetically effective because it creates a less prominent scar than that created by conventional laparoscopy.

Key-words: multichannel port, laparoscopy, adnexa.

\section{P17_4}

Comparison of postoperative pain grade by the number of ports after laparoscopic radical hysterectomy

S.H. Yoo, J.H. Yoon*

Department of Anaesthesiology, College of Medicine, Soon Chun Hyang University, Chunan, Korea; *Department of Obstetrics and Gynaecology, Catholic University Medical College, South Korea

Background: Laparoscopic surgery has many advantages compared with conventional methods and may allow a significant reduction in postoperative pain and analgesic consumption. Nevertheless, some patients still experience significant pain. Therefore, many clinicians have tried various methods to reduce of postoperative pain. We investigated degrees of postoperative pain by the different number of ports after laparoscopic radical hysterectomy.

Methods: Overall 75 patients were enrolled at this study and classified into 3 groups according to the number of ports used during the laparoscopic radical hysterectomy. The number of ports used for the operation varies from three to five. The intensities of abdominal were assessed 1, 6, 24 and 48 hours after surgery using a $100 \mathrm{~mm}$-horizontal visual analog scale (VAS) and a verbal rating scale (VRS). We also assessed the mean hospital stay for the three groups.

Results: The stage of cervical cancer patients is from 1A to IIA of FIGO. The mean age of 75 patients is $45.4 \pm 2.9$. The operation time was $212 \pm 8.7$ minutes and hospital stay was $9.8 \pm 4.1$. The mean VAS score of 75 patients was 4.3 (2177) $\mathrm{mm}$ and the mean VRS 5.2 (1-8). The abdominal pain 
scores (VAS and VRS) at 1 hour after surgery and the incidence of shoulder, epigastric, periumbilical pain and flank pain were significantly higher in patients group with 5 ports (mean VAS score; $64 \mathrm{~mm}$, VRS; 6.8) than any other groups for 1 hour after surgery $(\mathrm{P}<0.05)$.

Conclusions: During the laparoscopic radical hysterectomy, the larger number and diameter of trocars used during the operation is more painful against patient. So, the minimal use of trocars will be helpful for the patients.

Key-words: laparoscopic radical hysterectomy, pain grade, number of ports.

\section{P17_5}

Comparison of tissue effects quantified histologically between PlasmaJet coagulator and helica thermal coagulator

S. Deb, S. Deen, K.S. Ashford1, A. Harwood, C. Newman, M.C. Powell

Queen's Medical Centre, Nottingham University Hospitals NHS Trust, UK

Introduction: The PlasmaJet $(\mathrm{PJ})$ coagulator uses neutral pure argon plasma to achieve coagulation. Helica thermal coagulator (HTC) achieves coagulation with helium gas. HTC is currently used in the treatment of mild to moderate endometriosis.

Objective: The objective of this study was to compare the tissue damage caused by PJ to the HTC in the uterus, ovary, and fallopian tube. Our hypothesis was that PJ is a safe technology to use and that the tissue damage caused is comparable to HTC.

Methods: 15 subjects undergoing hysterectomy with or without salpingo-oophorectomy were prospectively recruited for in vivo assessment of the two instruments. Both instruments were used on a small area of uterus, ovary, and fallopian tube following the ligation of uterine artery pedicle. PJ was used at a power setting of $20 \%$ for duration of 5 seconds at a clinically acceptable distance of 0.5 to $1 \mathrm{~cm}$ from the tissue surface. HTC was used at a widely accepted low power setting used in the treatment of endometriosis for a similar duration and distance. Tissue damage was evaluated histologically. ANOVA was used to compare the mean differences.

Results: Data was normally distributed. 5 subjects had a subtotal hysterectomy and 10 had hysterectomy with salpingo-oophorectomy. A total of 15 uteri, 10 ovaries, and 10 fallopian tubes were histologically analysed for the tissue effect of PJ and HTC. There was no significant difference in the mean $\pm \mathrm{SD}$ depth of tissue damage seen between PJ and HTC in the uterus $(0.63 \pm 0.19$ vs $0.68 \pm$ $0.18 ; \mathrm{P}=0.481)$, ovary $(0.61 \pm 0.14$ vs $0.67 \pm 0.15 ; \mathrm{P}=0.420)$ and fallopian tube $(0.63 \pm 0.18$ vs $0.60 \pm 0.13 ; \mathrm{P}=0.688)$. A significantly lesser lateral spread of tissue damage (width) was seen with PJ than HTC in all three tissue types (uterus: $4.66 \pm 0.91$ vs $7.67 \pm 1.21, \mathrm{P}<0.001$; ovary: $4.05 \pm 0.61$ vs $5.90 \pm 0.95, \mathrm{P}<0.001$; fallopian tube: $4.50 \pm 0.77$ vs $6.00 \pm$ $1.28, \mathrm{P}=0.034)$.

Conclusions: The depth of tissue damage caused by PJ at $20 \%$ power is comparable to that with HTC on gynaecological tissues. The lateral spread (width of tissue damage) is however lesser with PJ than with HTC.

Key-words: PlasmaJet, Helica, coagulation.

\section{P17 6}

\section{A porcine model for the evaluation of thermal damage ex vivo and in vivo \\ R. Rothmund, B. Krämer, A. Reda, A. Neugebauer, D. Schäller \\ University Hospital for Women, Tübingen, Germany}

Summary: The experimental investigation of thermal damage in vital tissue is pivotal for the development of coagulation instruments. We present a porcine model to assess thermal spread in vivo and ex vivo. There was no significant difference in vivo and ex vivo regarding burst pressure and thermal damage.

Introduction: Thermal lateral damage as a result of electrocoagulation has mainly been tested in vivo in several animals. It remained unclear whether it is possible to obtain comparable results if the tissue is coagulated ex vivo.

Methods: We bilaterally coagulated the epigastric veins (2 coagulations/side) of $n=12$ pigs with two instruments (Maryland, ERBE and Kelly, KARL STORZ) after randomization in vivo and again 2 hours later after excision of the vessels (ex vivo). The thermal damage was investigated macroscopically, histopathologically and with an IR camera. The burst pressure was also investigated. The differences between in vivo and ex vivo were assessed.

Results: No significant difference between ex vivo and in vivo coagulation was found macroscopically, with IR and histologically. There was significantly more histopathological lateral damage compared to the macroscopic and IR findings. The focus was not on the comparison of the clamps, however lateral damage was non-significantly greater with the Kelly instrument.

Discussion: As the thermal damage was comparable in vivo and ex vivo, we postulate that it might be possible to use 
tissue from other experiments or cadavers, rather than using live animals in expensive settings to further improve electrocoagulation instruments.

Key-words: thermal damage, porcine model, electrocoagulation.

\section{P17_7}

\section{Non-bladed laparoscopic trocar system: a four year prospective study}

C. Zacharopoulou, A. Le Tohic, F. Renouvel, P. Panel

Mignot Hospital, Versailles, France

Objective: To evaluate the feasibility and safety of the direct trocar entry, using a non-bladed laparoscopic trocar system, to access the abdominal cavity during gynaecological interventions.

Materials and Methods: From January 2006 to June 2010, a total of 850 women have undergone to laparoscopic gynaecological operations in our hospital and they were included in this study.The technique requires a lateroumbilical incision, right or left, of about $15 \mathrm{~mm}$. With one hand we insert the trocar, perpendicular to the abdominal wall, until the fascia, whereas with the other hand we keep the abdominal wall lifted, to provide pressure against the force created by the trocar.. We twist the trocar through each layer. With the reduction of the resistance, we take out the cannula and we insert the laparoscope and we active gas insufflation.

Results: The mode of abdominal entry, patients demographics and complications were reviewed. The mean BMI was 24,3 (15-47). A transumbilical direct entry using a Taut ${ }^{\circledR}$ ADAPt ${ }^{\mathrm{TM}}$ device was used in 850 patients. Successful entry was obtained in 830 patients $(97,6 \%)$ whereas open laparoscopy was used in 18 women $(2,1 \%)$ after failure of the primary access with this device. Furthermore a Veress needle insertion technique was used in only 2 women $(0,23 \%)$. The mean duration of the procedure, between incision and insufflation, was 64 seconds, particularly $53 \mathrm{sec}$ by the senior and $84 \mathrm{sec}$ by the residents. No major complications were occurred, as vascular and bowel injuries. Two trocar-related injuries at the body of the uterus occurred in 2 patients $(0,26 \%)$. Conversion to laparotomy was not occurred. No insufflation-related complications such as gas embolism was observed.

Conclusion: Based in our results, we believe that the direct trocar technique with a non-bladed laparoscopic trocar system is feasible, safe, easy and rapid to perform. Although usually is a safe technique, a small minority of complications can occur. More research is required to define the safest technique.

\section{P17 8}

"Do it better and do it cheaper". Endobag for small specimens

I. Chatzipapas, A. Liapi, A. Protopapas, S. Ahanasiou, A. Antsaklis

$1^{\text {st }}$ University Department of Obstetrics and Gynaecology, Alexandra Hospital, Athens, Greece

Introduction: A potential problem during these procedures is the retrieval of the specimen, and usually, a laparoscopic bag is used for this purpose. Our objective is to introduce an inexpensive bag that achieves the same benefits but whose cost is low.

Methods: We have been experimenting with a urine specimen pediatric bag as an alternative to the Endobag. This bag is commonly used for accurate urine collection from non toilet trained children. These are easily obtainable from any Pediatric department. The largest size of the urine specimen pediatric bag is 5 by $7 \mathrm{~cm}$. The label on the bag facilitates the identification of the leaves of the mouth laparoscopically. The cost of the bag is very much less than the Endobag. In Greece, it costs only $0,15 €$. The operations were performed on patients under general anaesthesia with endotracheal intubation, and placed in the lithotomy position. The bladder was catheterized. Used the fourtrocar technique in each case. Once the bag insert in the abdomen the bag was unrolled and opened by separating the two leaves of both sides. The removed specimens were placed into the bag which was then closed by holding the two leaves together. The corner of the end of the bag was grasped with a pair of claw forceps, brought up to the abdominal incision by removing both the forceps and the port together.

Conclusions: The authors have several times used this bag without rupturing it. The use of urine specimen pediatric bag in gynaecological laparoscopy can easily remove several specimens without problem. The technique is simple, safe, fast and cost-effective. Most importantly, this bag is an inexpensive retrieval system which greatly reduces the total costs incurred by both hospital and patient. Key-words: endobag, specimens.

\section{P17 9}

An ancillary incision to remove an endobag with a surgical specimen larger than the trocar: A new technique

I. Chatzipapas, A. Protopapas, A. Liapi, S. Athanasiou, A. Antsaklis

$1^{\text {st }}$ University Department of Obstetrics and Gynaecology, Alexandra Hospital, Athens, Greece 
Introduction: Endobags are used to remove intra-abdominal surgical specimens during laparoscopic surgery. However, it is often difficult to extract the endobag through a $5-$ or $10-\mathrm{mm}$ cannula or the incision in the abdomen after removal of cannula due to the size of the specimen. Normally is performed by ancillary minilaparotomy, with enlargement of a trocar incision, or by other forms of laparotomy. We developed a new technique to facilitate delivery of the specimen through the abdominal wall incision with enlargement of a trocar incision using Farabeuf retractor.

Methods: An endobag is inserted through the suprapubic trocar $(10 \mathrm{~mm})$. The specimen is placed into it. The port is totally extracted, leaving the mouth of the bag exposed through the abdominal wall. Small retractors are introduced between the neck of the bag and the operative canal into the abdominal wall. A $1 \mathrm{~cm}$ skin incision is made with a scalpel in a cranium direction against the retractor. When the subcutaneous connective tissue is stretched apart, the fascia is incised in the longitudinal direction along the midline of the rectus fascia. The preperitoneal area is dissected, reaching the peritoneum, and its incision is extended enlarges the operative canal in the abdominal wall. We determined the direction of the tear of the anterior peritoneum by laparoscopic visualization. After this procedure, the bag can be reopened and the specimen extracted with forceps or morcellated inside the bag, or its contents can be aspirated. After removal of the endobag, the fascia is grasped under optical view and closed using an interrupted suture. Skin closing is routine.

Experience: We removed various sizes of specimens using this ancillary incision. In all cases, endobag removal with this ancillary incision was feasible. We observed no ruptures of endobag and closed the fascia in all cases after grasping the border under good visualization in order to decrease the incidence of incisional hernia.

Key-words: endobag, ancillary incision, surgical specimen

\section{Poster Session 18_Urogynaecology}

\section{P18_1}

Two stage surgical treatment of advanced pelvic organ prolapse in combination with stress urinary incontinence

I. Gladchuk, V. Marichereda, Y. Petrovskiy

Odessa State Medical University, Department of Gynaecology

n.1, Ukraine

Background: Pelvic organ prolapse(POP) is combined with stress urinary incontinence (SUI) in $20 \%$ of women older then 50 years. Currently there is no guidelines, describing how to manage this group of patients. Cases of "de novo" SUI and spontaneous SUI treatment after POP repair are described in multiple reports. Supplementing meshes for POP and SUI treatment is found to be more effective comparing with classical surgery . Aim of our research was to examine early and long-term results of two stage surgical treatment of POP in combination with SUI.

Materials and Methods: 485 consecutive POP repair cases were analysed. In 38 cases combined advanced POP and SUI required surgical treatment at the baseline. Each patient undergone POP-Q examination and urodynamical tests.

Results: Case histories of 38 patients with diagnosed combination POP and SUI in anamnesis were examined. Among them, 25,10 and 17 had defect of anterior, apical and posterior compartment defect respectfully. Patients undergone individual POP repair operation combination based on defectoscopic approach first with (18) or, then TVT-O procedure was performed if required. Among them, in 31 patients with POP and SUI combination were diagnosed initially and treated in two stages. Additionally 3 patients had occult SUI, that was diagnosed after POP repair. In 4 patients SUI was treated after POP repair spontaneously. Two patients had complications after POP surgery and 1 after SUI treatment. Conclusions: Two stage approach is a reasonable method of treating POP and SUI combination. More clinical trials comparing two and one stage approach are required.

Key-words: pelvic organ prolapse, stress urinary incontinence, surgical treatment

\section{P18_2}

Comparison of the early postoperative period using synthetic mesh or female tissues in surgical treatment of pelvic organs prolapse

G. Mečejus, E. Baužytė, A. Akelytė, R. Mušinskaitè Vilnius City University Hospital, Vilnius University Clinic of Obstetrics and Gynaecology, Lithuania

Introduction: Pelvic organ prolapse (POP) is common disorder that affects the quality of life up to $50 \%$ women in menopause. The wide variety of surgical treatments for POP using women tissues and lack of long-term success makes everybody look for new methods and technologies in POP treatment. Synthetic mesh for POP treatment is used to replace original female fascia for few years. But still now success rate and operation specific complications are different in literature data. The aim of this study is to compare early postoperative period in POP surgery using mesh with surgery, using female tissues.

Materials and Methods: Retrospective analysis of women case-records operated on POP from 2006 to 2008 in Vilnius 
City University Hospital, was done. All patients (190 patients) were divided into 2 groups: POP surgery with female tissues (1 group -130 patients) and POP surgery with mesh (2 group 60 patients). Women' age, duration of operation and hospitalization, medication intake, health disorders in the early postoperative period (within the first 3 days) were estimated. Results: Patient age and suffering time before surgery was same in the both groups. Duration of operation in 1 group was shorter than in 2 group $(54.1 \pm 21.4$ and $65.3 \pm 23.3$ minutes respectively). Duration of hospitalization and NSAID intake were less in the mesh group $(102.8 \pm 59.5$ and $71.9 \pm 48.9$ hours and $6.8 \pm 4.5$ and $4.12 \pm 2.9$ doses of NSAID in 2 and 1 group respectively). Early postoperative complications such as fever, urine retention and excessive bleeding were similar in both groups $(\mathrm{p}>0,05)$.

Discussion: POP surgery treatment using mesh has lower rate of NSAID intake and lower hospitalization time with the same rate of early postoperative complications in comparison with POP surgery treatment using female tissues. It seems to be a safe method in the early postoperation period with no additional problems. But there is still a need to continue the investigation in purpose to assess long-term effectiveness, late complications and remote results (after 1, 2 and 5 years) of the mesh operations.

Key-words: pelvic organs prolapse, mesh, early complications.

\section{P18_3}

Comparision of laparoscopic surgery outcomes in pelvic floor defects (cervicopexy vs hysteropexy).

M. Perelló, M. Gracia, C. Sisó, M. Espuña, F. Carmona

Department of Gynaecology, Hospital Clínic, Barcelona, Spain
Summary: The role that uterus plays in genital prolapses is still controversial. This study aims to compare laparoscopic hysteropexy and cervicopexy to establish the importance of the uterine preservation.

Introduction: Laparoscopic surgery offers similar results as abdominal surgery but with the advantages of a noninvasive technique. There is a lack of consensus about which is the best laparoscopic procedure, either preserve or not the uterus, for the management of pelvic floor defects.

Materials and Methods: From January 2006 to December 2009, 40 women with pelvic floor defects underwent surgery in Hospital Clínic with a laparoscopic technique: 10 hysteropexy and 30 cervicopexy. We did a retrospective comparison of demographic, clinical, physical,urodynamic and surgical data (SPSS V.17). The Baden-Walker system was used to grade the prolapse. The mean follow-up was 12 months.

Results: The mean operating time and blood loss is major in cervicopexy but without repercussion in hematologic parameters. No important surgery complications were reported.The mean hospital stay was 3 days. 39 women were satisfied at 6 months after surgery. 24 women consider a complete recuperation with no evidence of prolapse. 3 women with total cervical prolapse in the cervicopexy group were reoperated.

Discussion: Although outcomes do not show a significant statistically difference between both techniques, decreased time of surgery and blood loss in hysteropexy group, represents less morbidity and it is a feasible procedure to repair pelvic floor defects with a physiological restitution: preserving the uterus and total vaginal length.

Key-words: pelvic floor defect, cervicopexy, hysteropexy. 